\title{
2 Produkte zur Wundbehandlung
}

\subsection{Einführung}

\subsubsection{Voraussetzungen für eine ideale Wundauflage}

- physiologische und phasengerechte Wirkung $\rightarrow$ positive Beeinflussung der Wundheilung

- Aufrechterhaltung eines feuchten Wundklimas bei gleichzeitigem Aufrechterhalten des Gasaustausches (Sauerstoff, Kohlendioxid, Wasserdampf)

- ausreichendes Exsudataufnahmevermögen zur Vermeidung einer „feuchten“ Kammer oder einer Mazeration der Wunde bzw. Wundränder

- Beibehalten einer für die Wundheilung optimalen Temperatur

- kein Anhaften an den Wundgrund (atraumatischer Verbandwechsel)

- keine bis geringe Abgabe von Fasern, Partikeln oder zytotoxischen Substanzen in die Wunde, hypoallergen

- Verhinderung einer Keimbesiedlung der Wunde

- mechanischer Schutz der Wunde

- in geeigneten Größen und Formen lieferbar, ansonsten schneidbar.

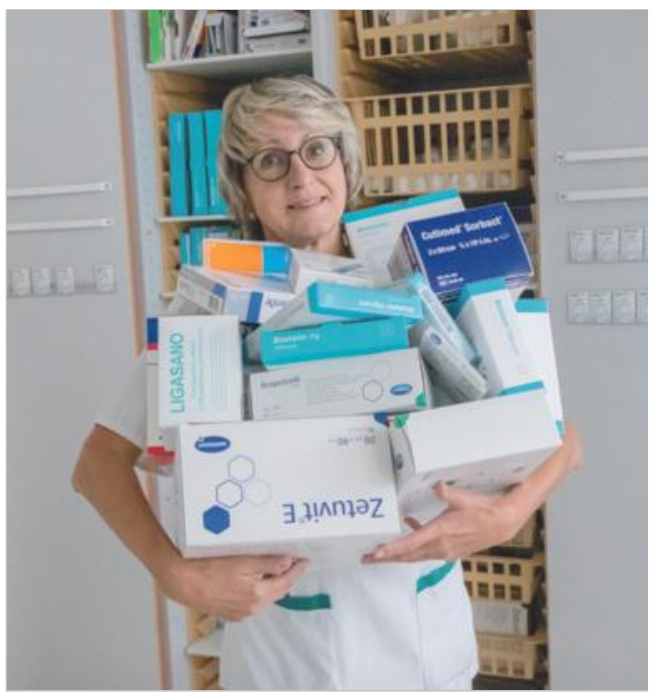

Abb. 2.1 Verschiedene Wundmaterialien.

(Quelle: Dorothee Sykora) 


\section{Merke}

Eine für alle Wundtypen und Heilungsphasen gleichermaßen geeignete Wundauflage gibt es nicht.

Das bisher schwer überschaubare Angebot von Wundauflagen wird im Folgenden detailliert vorgestellt. Es entspricht dem aktuellen Wissensstand von Experten aus unterschiedlichen Disziplinen. Es besteht kein Anspruch auf Vollständigkeit.

Die dargestellten Produkte wurden mit dem Fokus der Behandlung chronischer Wunden ausgewählt, damit sind weitere Präparate, wie z. B. Zyanoacrylat (Acrylatkleber), zur Behandlung primär zu schließender Wunden etc. nicht gesondert aufgeführt. Ebenso wird auf die Beschreibung von akuten Wunden verzichtet.

Es werden Therapievorschläge für die Behandlung einzelner Wundtypen gegeben.

Wundverbände bestehen grundsätzlich aus drei Komponenten ( $\triangleright$ Abb. 2.2):

- Wundfüller (Kissen), auf der Wunde aufliegend

- Adhäsivschicht zur Befestigung auf der umliegenden Haut

- Deckschicht als Schutz vor äußeren Einflüssen.

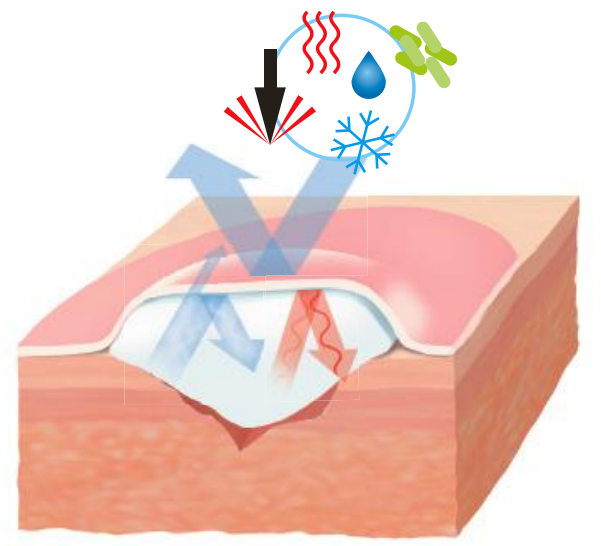

Abb. 2.2 Aufbau von Wundauflagen. 


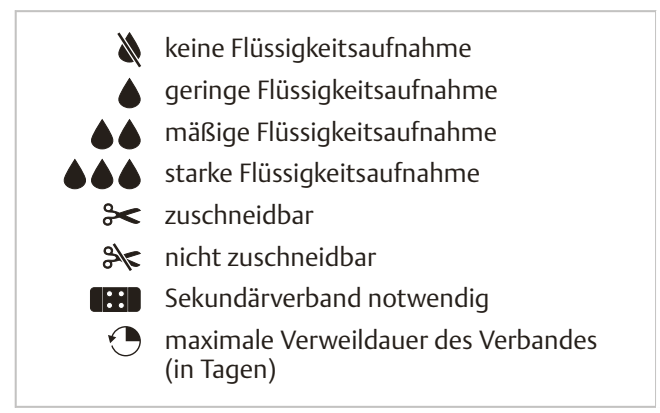

Abb. 2.3 Produkteigenschaften.

Wir unterscheiden Komplettsysteme, d. h. Wundauflagen, die alle drei Strukturen in einem Produkt vereinen, d. h. auch einen Abschluss bilden, von Wundprodukten, die sich lediglich als Wundfüller eignen und zusätzlich einen Abschluss nach außen, z. B. mit einem Hydropolymer, einem Hydrokolloid oder einer Polyurethanfolie, erfordern.

Im Folgenden werden zahlreiche auf dem Markt verfügbare Wundprodukte aufgeführt und näher erläutert.

Zunächst erfolgt die Darstellung der bei Helios eingeführten Produkte, gefolgt von vergleichbaren Produkten anderer Hersteller (beispielhaft, ohne Anspruch auf Vollständigkeit).

Anschließend werden die Produkte hinsichtlich Materialeigenschaften, Indikation, Anwendungsweise und Kontraindikation genauer beschrieben.

Die wesentlichen, in der Praxis relevanten Produkteigenschaften sind zusätzlich in Piktogrammen zusammengefasst. 


\subsection{Standardverbandmaterial}

\subsubsection{Kompressen}

\section{Gewobene Baumwollkompressen (steril)}

\section{Produkte}

\section{Gelistet in den Helios-Kliniken}

ES Kompressen (HAR), Telasorb (HAR, L\&R, MET):

- Mullkompresse St RK 12 -fach $10 \times 10 \mathrm{~cm}$ à 10 Stück

- Mullkompresse St RK 12 -fach $10 \times 10 \mathrm{~cm}$ à 20 Stück

- Mullkompresse St RK 12 -fach $10 \times 20 \mathrm{~cm}$ à 10 Stück

- Mullkompresse St RK 12 -fach $10 \times 20 \mathrm{~cm}$ à 20 Stück

- Mullkompresse St RK 32 -fach $10 \times 10 \mathrm{~cm}$ à 10 Stück

- Mullkompresse St RK 16-fach $5 \times 5$ cm à 10 Stück

- Verbandmull $80 \mathrm{~cm}$ breit $200 \mathrm{~m}$

- Verbandmull 8-fach Zickzack 5 m

- Mullkompressen St 12-fach $10 \times 10 \mathrm{~cm}$ à 5 Stück

- Mullkompressen St 12-fach $10 \times 20 \mathrm{~cm}$ à 5 Stück

- Bauchtuch St 6 -fach $45 \times 45 \mathrm{~cm}$ weiß à 5 Stück

- Bauchtuch St 4-fach $20 \times 30 \mathrm{~cm}$ weiß à 5 Stück

- Bauchtuch St 6 -fach $45 \times 45 \mathrm{~cm}$ weiß à 2 Stück

- Bauchtuch St 4-fach $90 \times 8 \mathrm{~cm}$ weiß à 2 Stück

- Bauchtuch St 6-fach $45 \times 45 \mathrm{~cm}$ grün à 2 Stück

- Bauchtuch St 4 -fach $45 \times 45 \mathrm{~cm}$ grün à 5 Stück

\section{Beschreibung}

- Mull (Übersicht s. $>$ Abb. 2.4) ist ein Gewebe aus Baumwolle, das je nach Fadendicke eine feine oder grobe Gitterstruktur aufweist.

- Die Kompressen sind so gefaltet, dass Schnittkanten nach innen gelegt werden.

- Die Saugstärke wird von der Dicke der Kompressen bestimmt, 4-, 8- ,12- ,16-, 32-fache Lagen.

- Ab 16 oder mehr Lagen wird das Wundexsudat dreidimensional in obere Kompressenlagen abgeleitet.

- Keine Keimbarriere. 


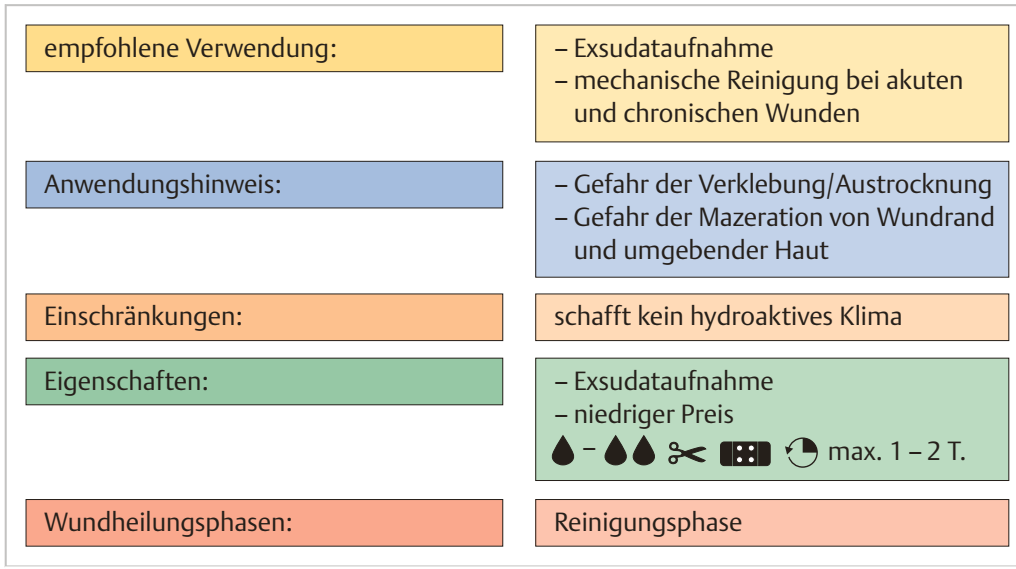

Abb. 2.4 Standardverbandmaterial: gewobene Baumwollkompressen.

\section{Indikation}

- geeignet zur mechanischen Reinigung der Wunde durch raue Gitterstruktur

- zur Erstversorgung von verschmutzten, blutenden oder stark exsudierenden Wunden $\rightarrow$ Saugkraft; Ableiten von Exsudat, nekrotischem Gewebe, Bakterien (Löschblatteffekt)

- Trägermaterial für Antiseptika etc.

- Standardversorgung von akuten Wunden, z. B. postoperativ nach primärem Wundverschluss.

\section{Kontraindikation}

Ungeeignet in der Granulations- und Epithelisierungsphase (hier besser Wundruhe mit geeigneter Abdeckung).

\section{Anwendungsweise}

- Wundkontakt ist sicherzustellen

- bei schwach exsudierenden Wunden Austrocknungs- und Verklebungsgefahr. 


\section{Cave}

Bei Überlappung über den Wundrand Gefahr der Mazeration der umgebenden Haut.

Sekundärabdeckung: notwendig.

Verbandwechsel:

- sobald der Verband durchfeuchtet ist

- häufiger Verbandwechsel 1-2 Tage, ggf. auch mehrmals täglich.

\section{Vlieskompressen (steril)}

\section{Produkte}

\section{Gelistet in den Helios-Kliniken}

BeeSana Vlieskompresse (MET):

- $5 \times 5 \mathrm{~cm}$ steril à 2 Stück

- $10 \times 10 \mathrm{~cm}$ steril à 5 Stück

- $10 \times 20 \mathrm{~cm}$ steril à 2 Stück

- Schlitzkompresse Vlies St 7,5×7,5 cm à 2 Stück

- Schlitzkompresse Vlies St $10 \times 10 \mathrm{~cm}$ à 2 Stück.

\section{Beschreibung}

- Vliesstoffe (Übersicht s. - Abb. 2.5) sind durch mechanische oder chemische Verfestigung hergestellte Textilien (nicht gewebt/non-woven).

- Sie bestehen aus synthetischen Fasern wie Polyamid, Polyester, Polypropylen, Zellwolle (Viskose), seltener aus ungewebter Baumwolle oder auch aus Fasermischungen.

- Sie sind deutlich preiswerter als gewebte Baumwollkompressen.

- Ihre Saugkapazität ist nur sehr gering.

- Sie bilden keine Keimbarriere. 


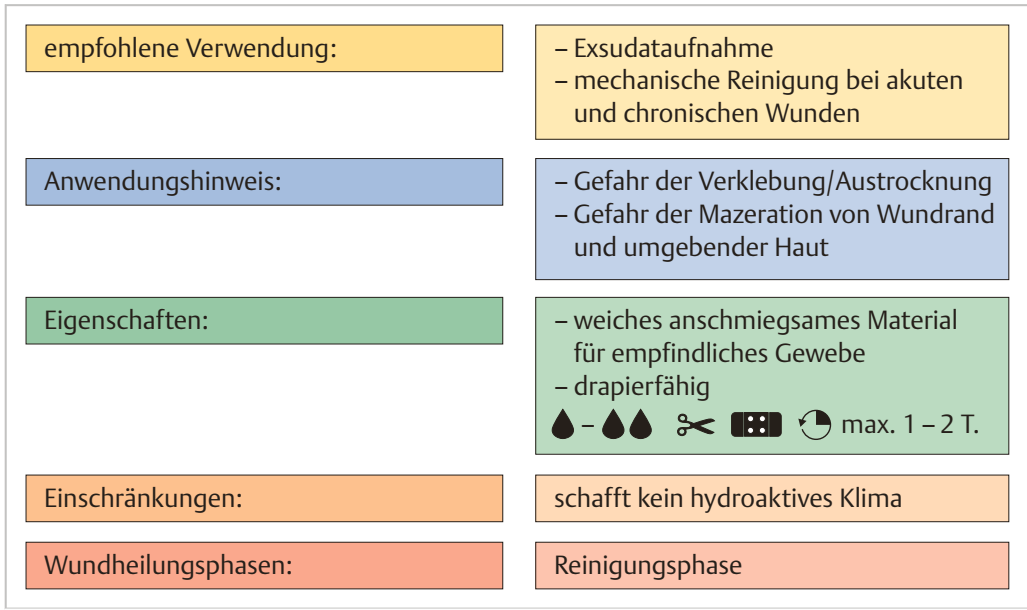

Abb. 2.5 Standardverbandmaterial: Vlieskompressen.

\section{Indikation}

- zur Erstversorgung von verschmutzten, blutenden oder stark exsudierenden Wunden $\rightarrow$ Saugkraft; Ableiten von Exsudat, nekrotischem Gewebe, Bakterien (Löschblatteffekt)

- Standardversorgung von akuten traumatischen Wunden und postoperativ

- zur Reinigung akuter und chronischer Wunden.

\section{Kontraindikationen}

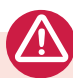

- ungeeignet in der Granulations- und Epithelisierungsphase (besser Wundruhe mit geeigneter Abdeckung)

- bei schwach exsudierenden Wunden Austrocknungs- und Verklebungsgefahr. 


\section{Anwendungsweise}

Wundkontakt ist sicherzustellen.

\section{Cave}

Bei Überlappung über den Wundrand Gefahr der Mazeration der umgebenden Haut.

Sekundärabdeckung: notwendig.

Verbandwechsel:

- sobald der Verband durchfeuchtet ist

- häufiger Verbandwechsel 1-2 Tage, ggf. auch mehrmals täglich.

\subsubsection{Saugkompressen}

\section{Saugkompressen ohne Superabsorber}

\section{Produkte}

\section{Gelistet in den Helios-Kliniken}

Zetuvit E (HAR) $10 \times 10 \mathrm{~cm}, 10 \times 20 \mathrm{~cm}, 20 \times 20 \mathrm{~cm}, 20 \times 40 \mathrm{~cm}$ ( $\triangleright$ Abb. 2.6).

\section{Vergleichbare Produkte}

- Askina Pad (BBR)

- Cutisorb (BSN)

- Draco Saugkompressen (DRA)

- Ete (MÖL)

- Melolin (S\&N)

- Noba Saugkompresse (NOB)

- Solvaline N, Vliwacell, Vliwin (L\&R)

- Telfa, Telfa AMD, Telfa Max (COV)

- Urgo Pad (URG)

- Zetuvit (HAR). 


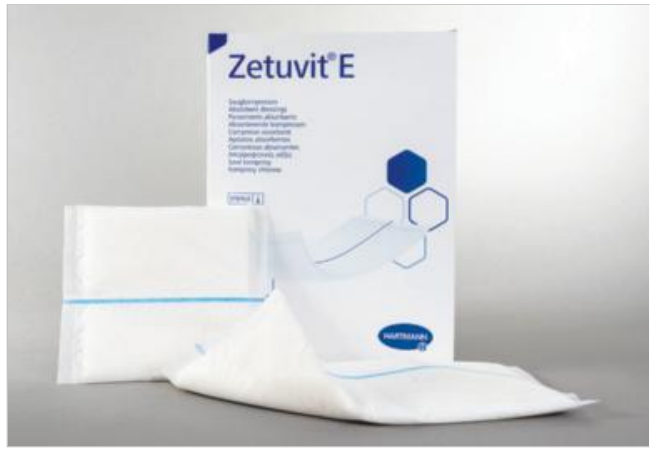

Abb. 2.6 Zetuvit E.

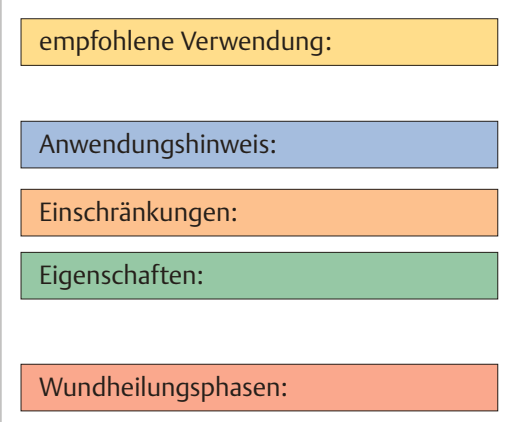
mittelgradig bis stark nässende Wunden

weiße Seite auf Wunde

trockene Wunden

- hohe Saugwirkung

sk a: $1-3, \max 5 \mathrm{~T}$.

Reinigungsphase/Granulationsphase

Abb. 2.7 Standardverbandmaterial: Saugkompressen.

\section{Beschreibung}

Saugkompressen ohne Superabsorber (Übersicht s. \ Abb. 2.7) bestehen aus mehreren Materialschichten mit hochsaugfähigem Kern aus Zellstoffflocken oder Watte und einer Umhüllung, die aus einem glatten, wundfreundlichen Vliesstoff (meistens aus hydrophoben synthetischen Fasern) hergestellt ist.

Einige Produkte sind auf der Rückseite mit einer feuchtigkeitsabweisenden Schicht ausgestattet, um ein Durchfeuchten zu vermeiden. 


\section{Indikation}

- zur Versorgung stark exsudierender Wunden

- Sekundärverband: als Abdeckung von Primärverbänden (z. B. Alginat) oder als Kombination Polsterung/Saugkomponente.

\section{Kontraindikation}

Schwach bis mäßig exsudierende Wunden in der Granulations- und Epithelisierungsphase.

\section{Anwendungsweise}

- Zweidimensionales Weiterleiten von Exsudat; auf Mazeration des Wundrandes und der umgebenen Haut achten.

- Plan auf die Wunde legen, mit Binden, Pflasterstreifen oder Klebevlies fixieren.

- Die feuchtigkeitsabweisende Seite nach außen legen (meistens gekennzeichnet durch Farbe oder Symbole), weiße Seite zur Wunde.

\section{Merke}

Zetuvit E darf nicht zugeschnitten werden!

\section{Sekundärabdeckung:}

- notwendig

- Fixomull, Folie, Pflasterstreifen, Mullbinde.

Verbandwechsel: sobald der Verband durchfeuchtet ist, ggf. mehrmals täglich. 


\section{Saugkompressen mit Superabsorber}

\section{Produkte}

\section{Gelistet in den Helios-Kliniken}

- RespoSorb Super (HAR) $10 \times 10 \mathrm{~cm}, 10 \times 20 \mathrm{~cm}, 20 \times 25 \mathrm{~cm}, 20 \times 40 \mathrm{~cm}$ ( $\triangleright$ Abb. 2.8)

- Eclypse Contour $30 \times 51 \mathrm{~cm}$, Eclypse Boot $55 \times 47 \mathrm{~cm}, 60 \times 70 \mathrm{~cm}, 70 \times 80 \mathrm{~cm}$, Eclypse Foot $33 \times 48 \mathrm{~cm}(\mathrm{ADV})$ alle partiell zuschneidbar.

\section{Vergleichbare Produkte}

- Askina Absorb + (BBR)

- Curea P1 (CUR)

- Cutisorb Ultra, Cutimed Sorbion Sachet Border, Cutimed Sorbion Sachet Extra, Sorbion Sachet Multi Star, Cutimed Sorbion Sachet S, Cutimed Sorbion sana, Cutimed Sorbion Sachet XL (BSN)

- DryMax extra (MDH)

- Duramax, Exu-Dry, Allevyn Life (S\&N)

- Mextra Superabsorbent (MÖL)

- ROGG Sorb lite (ROG); ist zuschneidbar

- Tegaderm Superabsorber (3M)

- UrgoSuperabsorber, UrgoSuperSuperabsorber (URG)

- Vliwasorb Pro (L\&R)

- Zetuvit plus (HAR).

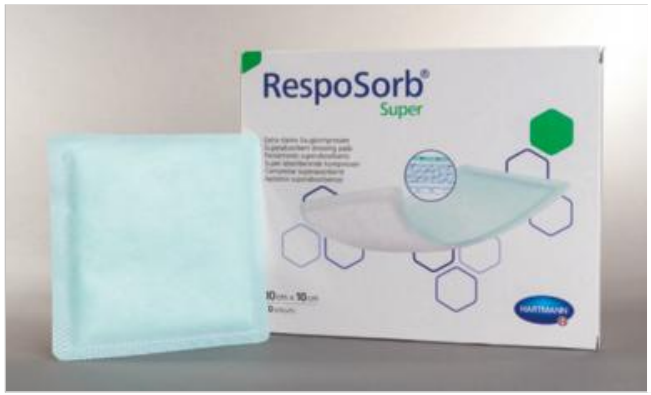

Abb. 2.8 RespoSorb Super. 


\section{Beschreibung}

Saugkompressen mit Superabsorber unterscheiden sich lediglich durch eine stark erhöhte Exsudataufnahmefähigkeit von den übrigen Saugkompressen. Sie eignen sich daher besonders unter Kompressionsverbänden und Kompressionsstrümpfen, da das Exsudat im Superabsorber gebunden bleibt und nicht unter Druck in die Wunde gepresst wird (siehe auch Kap. 2.8).

\section{Indikation}

- Zur Versorgung sehr stark exsudierender Wunden. Auch geeignet als Wundverband unter Kompressionsverbänden.

- Sekundärverband: als Abdeckung von Primärverbänden (z. B. Alginat) oder als Kombination Polsterung/Saugkomponente.

\section{Kontraindikation}

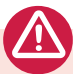

Schwach bis mäßig exsudierende Wunden in der Granulations- und Epithelisierungsphase.

\section{Anwendungsweise}

- Zweidimensionales Weiterleiten von Exsudat; auf Mazeration des Wundrandes und der umgebenen Haut achten.

- Plan auf die Wunde legen, mit Binden, Pflasterstreifen oder Klebevlies fixieren.

- Die feuchtigkeitsabweisende Seite nach außen legen (meistens gekennzeichnet durch Farbe (grün) oder Symbole), weiße Seite zur Wunde.

\section{Cave}

RespoSorb Super darf nicht zugeschnitten werden!

\section{Sekundärabdeckung:}

- notwendig

- Fixomull, Folie, Pflasterstreifen, Mullbinde.

Verbandwechsel: sobald der Verband durchfeuchtet ist, ggf. mehrmals täglich. 


\subsubsection{Fixiermittel}

\section{Semipermeable Folienverbände}

\section{Produkte}

\section{Gelistet in den Helios-Kliniken}

- Xtrata Perme-Roll (unsterile) (MED) $5 \mathrm{~cm} \times 10 \mathrm{~m}, 10 \mathrm{~cm} \times 10 \mathrm{~m}, 15 \mathrm{~cm} \times 10 \mathrm{~m}$, Wasserdampfdurchlässigkeit: $1100 \mathrm{~g} / \mathrm{m}^{2} / 24 \mathrm{~h}$ ( $\triangleright$ Abb. 2.9, $\triangleright$ Abb. 2.10)

- $3 \mathrm{M}$ Tegaderm Film Transparentverband (steril) $(3 \mathrm{M}) 6 \times 7 \mathrm{~cm}, 10 \times 12 \mathrm{~cm}$, $10 \times 25 \mathrm{~cm}, 15 \times 20 \mathrm{~cm}, 20 \times 30 \mathrm{~cm}$ ( $\vee$ Abb. 2.11$)$

- Leukomed T (steril) (BSN) 7,2 $\times 5 \mathrm{~cm}, 10 \times 12,5 \mathrm{~cm}, 10 \times 25 \mathrm{~cm}, 15 \times 20 \mathrm{~cm}$, $15 \times 25 \mathrm{~cm}$, Wasserdampfdurchlässigkeit: $900 \mathrm{~g} / \mathrm{m}^{2} / 24 \mathrm{~h}$

- Mepitel Film (MÖL) $10 \times 12 \mathrm{~cm}, 10 \times 25 \mathrm{~cm}$, Wasserdampfdurchlässigkeit: $904 \mathrm{~g} / \mathrm{m}^{2} / 24 \mathrm{~h}$ ( $\vee$ Abb. 2.12).

\section{Nicht gelistet}

- Opsite Flexfix - unsterile Rolle (S\&N)

- Opsite Flexfix Gentle (S\&N)

- Opsite Flexgrid (S\&N)

- Bioclusive Plus - transparenter Folienverband (SYS)

- Nobaderm - transparenter Folienverband (NOB)

- Mepore Film unsteril (MÖL), Mepore Film steril (MÖL), Mepore Film mit Savetac-Technologie steril (MÖL)

- Leukomed T (BSN), Fixomull transparent unsteril (BSN)

- Kendall Transparenter Folienverband (COV)

- Hydrofilm (HAR)

- Optiskin Film steril (URG), Optiskin Film Roll unsterile (URG)

- Suprasorb F Folien - Wundverband (L\&R).

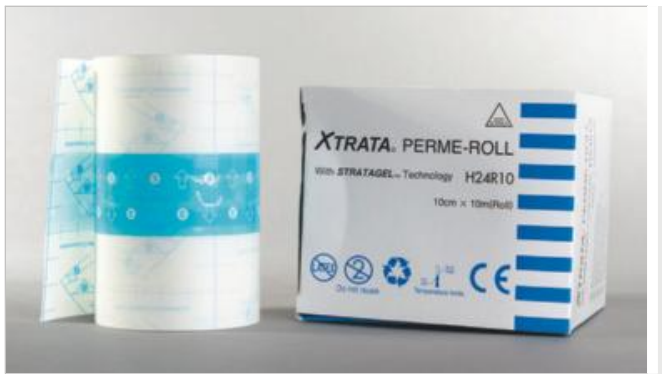

Abb. 2.9 Xtrata (unsteril). 


\section{Wundprodukte}

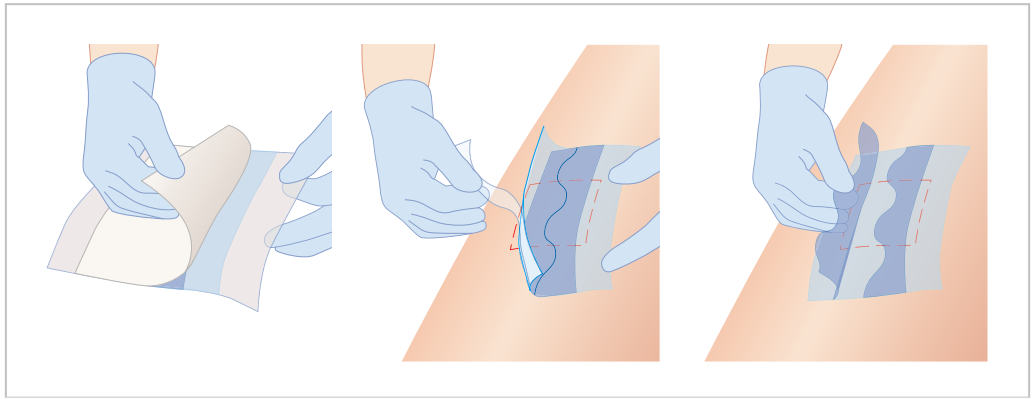

Abb. 2.10 Xtrata: Anwendung.

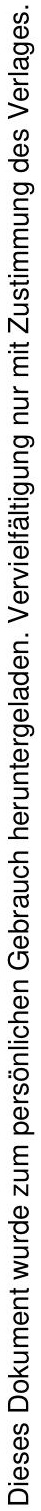




\section{Beschreibung}

Semipermeable Wundfolien (Übersicht s. $>$ Abb. 2.13) sind selbsthaftende, bakterien- und wasserdichte, transparente Polyurethanfolien, die durchlässig sind für Luft, Sauerstoff und Wasserdampf (Duschen mit Verband möglich).

Die meisten bei Helios gelisteten semipermeablen Wundfolien haben eine mäßige Wasserdampfdurchlässigkeit ( $<1000 \mathrm{ml} \mathrm{H}_{2} \mathrm{O} / \mathrm{m}^{2}$ in $\left.24 \mathrm{~h}\right)$. Sie sind daher nicht für eine mehrtägige Dauerabdeckung geeignet. Dies gilt insbesondere bei starker Transsudation wie bei Fieber, offenem Abdomen, Wundinfekten etc.

Semipermeable Wundfolien (z.B. Xtrata) mit hoher Durchlässigkeit ( $>1000 \mathrm{ml} \mathrm{H} \mathrm{H}_{2} \mathrm{O} / \mathrm{m}^{2}$ in $24 \mathrm{~h}$ ) erlauben eine längere Liegedauer, sind aber mechanisch instabiler (dünner!) und erfordern auch tägliche Wundkontrollen. Es ist wichtig, Mazerationen zu vermeiden, da sonst eine erhöhte Infektionsgefahr besteht.

Der Vorteil von Folien ist die Möglichkeit der Wundbeurteilung ohne Verbandentfernung!

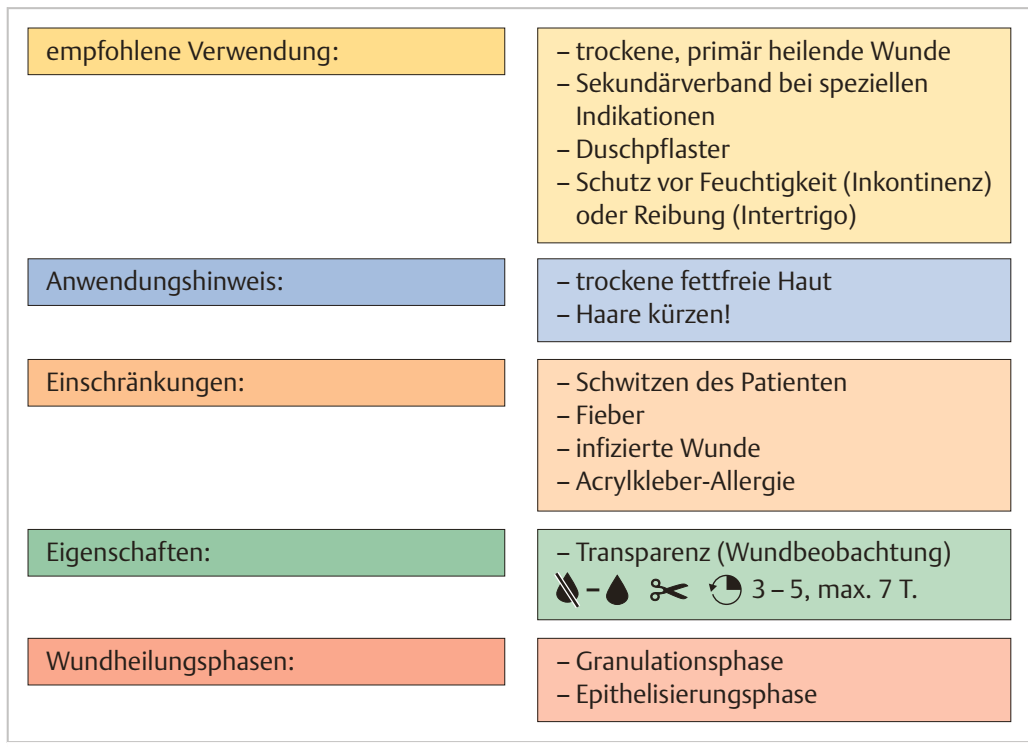

Abb. 2.13 Fixiermittel: semipermeable Folien. 


\section{Indikation}

Folien mit geringer Durchlässigkeit:

- als Abdeckung über Wundprodukten, die einen Sekundärverband benötigen

- für trockene, primär heilende Wunden

- zur kurzfristigen Okklusion (z. B. bei Anwendung von LokalanästhetikaCremes, wie z. B. Emla-Creme)

- Okklusionsverbände bei Fingerkuppenverletzungen Typ Allen III und IV - hier längere Verbandwechselintervalle! Der primäre Okklusionsverband wird so lange wie möglich belassen und, wenn nötig, nur abgedichtet. - selbst bei einem über das Wundniveau hinausstehenden Knochen kann eine zufriedenstellende Weichteildeckung erzielt werden

- zur Abdichtung (Vakuumverband)

- als Duschpflaster ( $\triangleright$ Abb. 2.14).

Folien mit hoher Durchlässigkeit:

- als Abdeckung über Wundprodukten, die einen Sekundärverband benötigen

- zur Fixierung von Kathetern und Kanülen

- als Schutz der Haut vor Feuchtigkeit (Inkontinenz) oder Reibung (Intertrigo).

\section{Kontraindikationen}

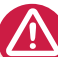

- Die alleinige Anwendung von Folien ist nur bei nicht exsudierenden Operationswunden zulässig.

- Bei pAVK darf eine Folie nur eingesetzt werden, wenn eine tägliche Beobachtung gewährleistet ist.

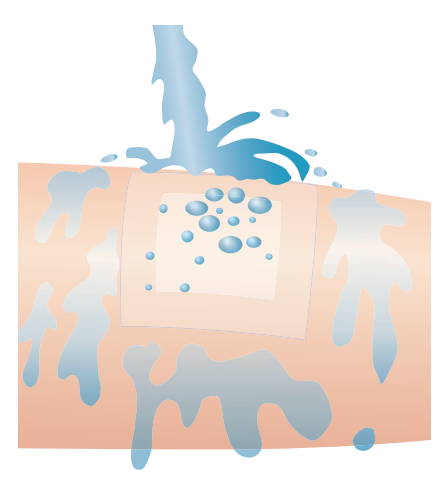

Abb. 2.14 Xtrata als Duschpflaster. 


\section{Anwendungsweise}

Entfernen der Folie atraumatisch durch Überdehnung des Verbandes möglich.

Sekundärabdeckung: keine.

Verbandwechsel: alle 1-7 Tage.

\section{Besonderer Einsatz von Folienverbänden: Regeneration von Fingerkuppen nach Amputationsverletzungen}

Die häufigste Verletzung der Finger ist die Amputationsverletzung an den Fingerendgliedern. Die Behandlung ist oft eine operative mit lokalen Lappenplastiken. Alternativ können jedoch mit einem semiokklusiven Folienverband sehr gute funktionelle und ästhetische Resultate erreicht werden ( $\triangleright$ Abb. 2.15). Selbst freiliegender Knochen ist keine Kontraindikation für eine Folienbehandlung. Amputationsverletzungen distal der Insertion der Beugesehne (Zonen I-III nach Ishikawa) sind mit Folienverbänden behandelbar.

\section{Vorgehen:}

- keine Manipulation am Stumpf

- keine Nagelbettnaht

- kein Anlegen eines Kunstnagels

- keine Weichteilresektion

- ggf. Kürzen des überstehenden Knochens mit einer Schere auf das Weichteilniveau

- Reinigung und Inspektion der Wunde am Unfalltag in Lokalanästhesie

- ggf. Röntgen

- keine Eradikation des Restnagels

- Behandlung mit der Folie am Unfalltag; späterer Beginn ist notfalls möglich.

Anlage des Verbandes:

- Folie direkt auf die Wunde legen; ca. $2 \mathrm{~cm}$ unter Amputationslinie

- keine Manipulation der Wunde

- keine Desinfektion

- Koagel und schmierige Beläge belassen

- Auslaufen von trüber und übelriechender Flüssigkeit ist normal $\rightarrow$ kein Grund zum Verfahrenswechsel

- Haut unter der Folie mazeriert

- Trocknen der Haut unmittelbar proximal der Amputationslinie, Wundfläche selber muss feucht bleiben!

- Anlage neuer Verband

- Sekundärverband über die Folie zum mechanischen Schutz und zum Auffangen von Wundexsudat. 

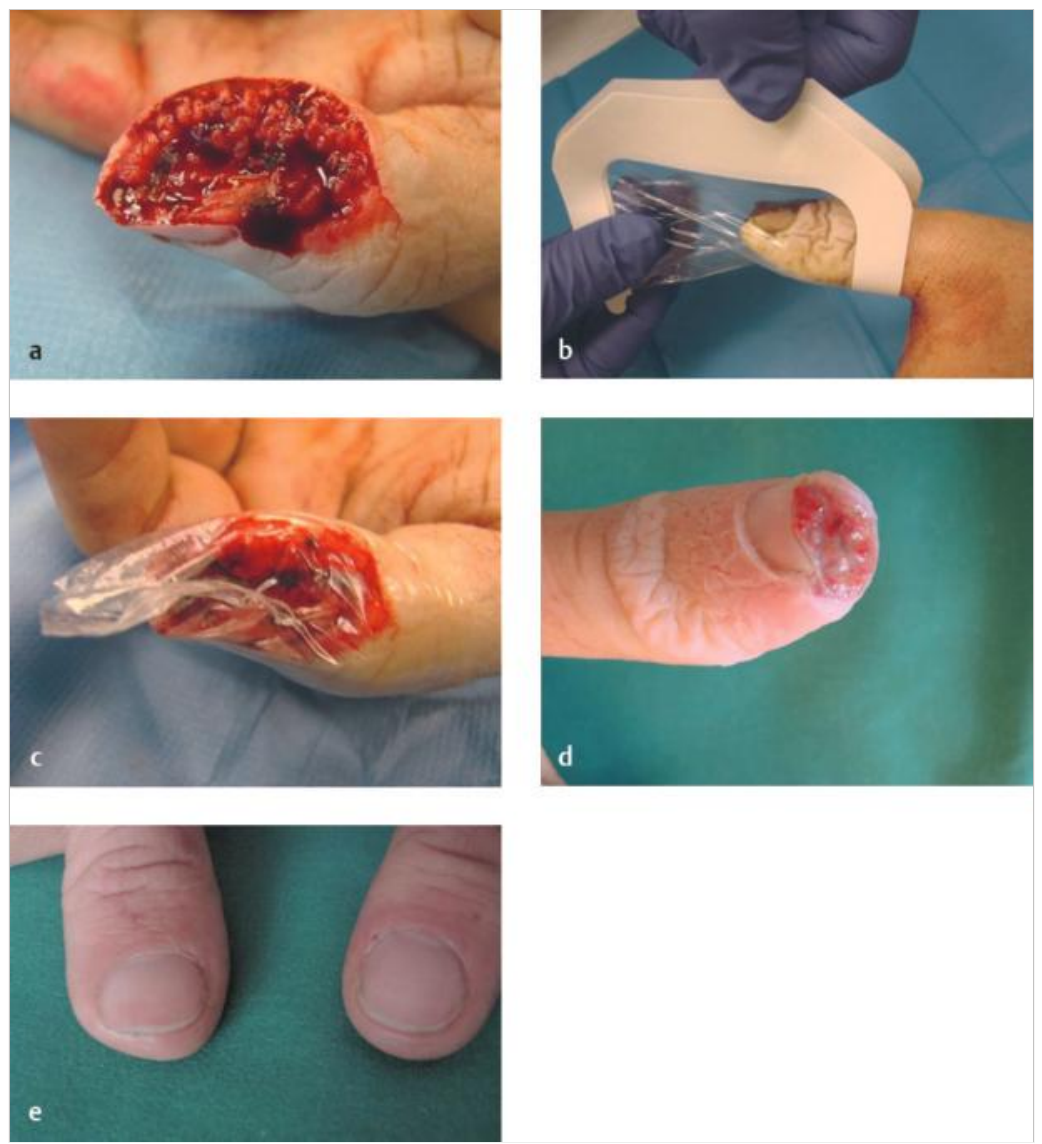

Abb. 2.15 Folienverband bei Fingerkuppenamputation. a-e

\section{Cave}

Der Verband über der Folie muss locker sein, sonst kommt es zu Verformungen des Gewebes. 
Nach 3 Wochen deutliche geringere Berührungsempfindlichkeit, dann ist ein dünnerer Schutzverband möglich.

Verbandwechsel: wöchentlich.

Geruchsemission: Diese entwickelt sich innerhalb von 1-2 Wochen. Um entgegenzuwirken, muss man die Haut beim wöchentlichen Folienwechsel gut reinigen. Der Finger darf auch mit sterilem Wasser/NaCl-Lösung gereinigt werden. Weiterhin keine Manipulation und keine Desinfektion an der Wundfläche. Wechsel des Schutzverbandes täglich. Alternativ über der Folie eine Schicht Aktivkohle anbringen.

Dauer der Behandlung:

- Die Epithelialisierung ist erst beendet, wenn kein Exsudat mehr austritt.

- Bei trockenen Verhältnissen z. B. komplettem Anhaften der Folie auf dem ganzen Endglied ohne Abgleiten gibt es keinen Grund mehr für die Folienbehandlung.

- Dauer der Epithelialisierung, je nach Größe des Defektes ca. 3-7 Wochen.

- Das Nagelbett epithelialisiert unter der Folie nicht. Kein Abwarten des Nachwachsens des Nagels. Nach Beendigung der Folienbehandlung normalisiert sich das Gewebe von selbst.

\section{Fixiermittel für normal belastbare Haut}

\section{Produkte}

\section{Gelistet in den Helios-Kliniken}

Fixomull stretch ( $\triangleright$ Abb. 2.16); $5 \mathrm{~cm} \times 20 \mathrm{~m}, 10 \mathrm{~cm} \times 20 \mathrm{~m}, 15 \mathrm{~cm} \times 20 \mathrm{~m}$, $20 \mathrm{~cm} \times 20 \mathrm{~m}, 30 \mathrm{~cm} \times 20 \mathrm{~m}$ (BSN).

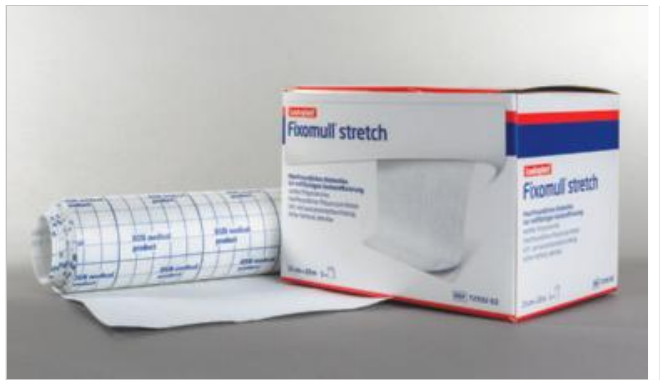

Abb. 2.16 Fixomull stretch. 


\begin{tabular}{|l|}
\hline empfohlene Verwendung: \\
\hline Anwendungshinweis: \\
\hline
\end{tabular}

Eigenschaften:

\section{Einschränkungen:}

Besonderheit: normal belastbare Haut

- kann nur einmalig positioniert werden

- Cave: Gefahr des Strip-off-Effektes (Entfernung der obersten Epidermiszelllagen aufgrund der starken Klebehaftung)

- Altershaut

- Pergamenthaut

- Neugeborenen-/Frühgeborenenhaut

- Dialysepatienten

- medikamentös beanspruchte Haut

- strahlengeschädigte Haut

- sehr fest haftender Polyacrylkleber zur dauerhaften Fixierung

d) $3-5, \max .7 \mathrm{~T}$.

zur vollflächigen Fixierung von Wundauflagen, insbesondere an Gelenken, bewegten und konturierten Körperpartien

Abb. 2.17 Fixiermittel für normal belastbare Haut.

\section{Beschreibung}

Fixomull stretch dient als Standardprodukt zur Befestigung von Verbänden, Sonden, Kathetern etc. bei normal belastbarer Haut. Es handelt sich um ein atmungsaktives, flexibles Trägermaterial aus weichem Vlies mit Polyacrylat-Haftung (Übersicht s. > Abb. 2.17).

\section{Indikation}

Für normal belastbare Haut. 


\section{Kontraindikationen}

- Altershaut

- Pergamenthaut

- Neugeborenen-, Frühgeborenenhaut

- Dialysepatienten

- medikamentös beanspruchte Haut

- strahlengeschädigte Haut.

\section{Anwendungsweise}

- kann an konturierten Körperstellen appliziert, aber danach nicht mehr neu positioniert werden ( $\vee$ Abb. 2.18)

- eine Tragedauer ist bis zu 7 Tage möglich

- geeignet als zusätzliche Fixierung von u. a. Sonden, Tuben, Kathetern

- auf Rolle, ist bedarfsgerecht zuschneidbar

- indikationsgerechter Einsatz bei niedrigen Kosten.

Sekundärabdeckung: nicht notwendig.

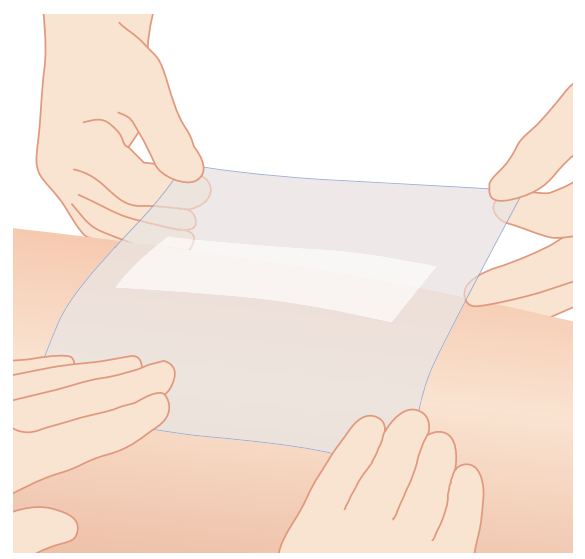

Abb.2.18 Fixomull stretch: Anwendung an konturierten Körperstellen. 


\section{Fixiermittel für empfindliche Haut}

\section{Produkte}

\section{Gelistet in den Helios-Kliniken}

Fixomull skin sensitive (BSN) $5 \mathrm{~cm} \times 5 \mathrm{~m}$ ( $\triangleright$ Abb. 2.19).

\section{Vergleichbares Produkt}

Silikonbeschichtetes Pflaster $5 \mathrm{~cm} \times 5 \mathrm{~m}(3 \mathrm{M})$.

Übersicht s. \ Abb. 2.20

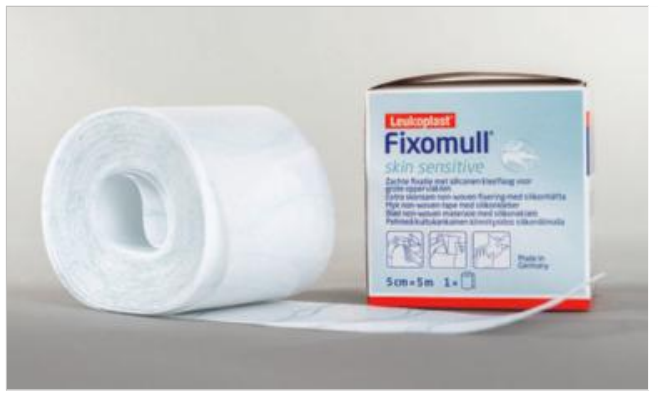

Abb. 2.19 Fixomull skin sensitive.

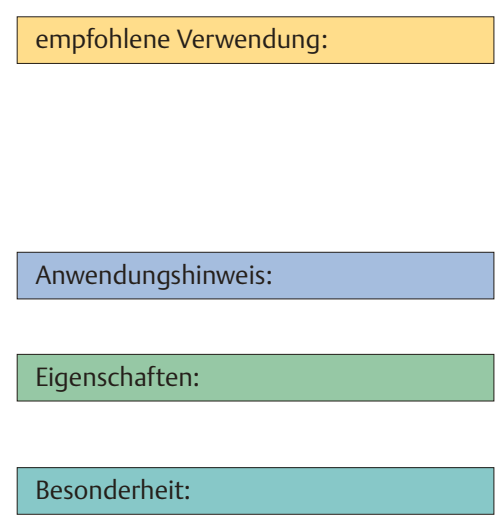

für besonders empfindliche Haut:

- Altershaut

- Pergamenthaut z.B. durch Steroide

- Neugeborenen-/Frühgeborenenhaut

- Dialysepatienten

- strahlengeschädigte Haut

kann mehrmals neu positioniert werden

- atraumatisch

$\|$ \& $3-5, \max .7 \mathrm{~T}$.

- nur bei extrem empfindlicher Haut

- kein Standardprodukt

Abb. 2.20 Fixiermittel für empfindliche Haut. 


\section{Beschreibung}

- Fixomull skin sensitive dient wie Fixomull stretch zur Befestigung von Verbänden, Sonden, Kathetern etc. bei besonders empfindlicher Haut. Hervorzuheben ist die atraumatische Entfernbarkeit.

- Atmungsaktives, flexibles Trägermaterial aus weichem Vlies mit Silikonhaftung.

\section{Cave}

Standardprodukt bei normaler Haut ist Fixomull stretch.

\section{Indikation}

Für besonders empfindliche Haut wie z. B.

- Altershaut

- Pergamenthaut

- Neugeborenen-, Frühgeborenenhaut

- Dialysepatienten

- medikamentös beanspruchte Haut

- strahlengeschädigte Haut.

\section{Kontraindikationen}

Keine.

\section{Anwendungsweise}

- kann an konturierten Körperstellen appliziert und mehrmals neu positioniert werden

- eine Tragedauer ist bis zu 7 Tage möglich

- geeignet als zusätzliche Fixierung von u. a. Sonden, Tuben, Kathetern

- auf Rolle, ist bedarfsgerecht zuschneidbar

- nur bewusster indikationsgerechter Einsatz bei ökonomisch hohen Kosten.

Sekundärabdeckung: nicht notwendig. 


\subsubsection{Distanzgitter (Gaze)}

\section{Produkte}

\section{Distanzgitter}

Gelistet in den Helios-Kliniken

- Atrauman (HAR) $7,5 \times 10 \mathrm{~cm}, 10 \times 20 \mathrm{~cm}(\triangleright$ Abb. 2.21)

- Cuticerin (S\&N) 7,5 ×7,5cm, 7,5 $\times 20 \mathrm{~cm}(\triangleright$ Abb. 2.22)

- Grassolind (HAR) $5 \times 5 \mathrm{~cm}, 10 \times 10 \mathrm{~cm}, 10 \times 20 \mathrm{~cm}$ ( $\vee$ Abb. 2.23)

- Jelonet (S\&N) Paraffingazeverband $5 \times 5 \mathrm{~cm}, 10 \times 10 \mathrm{~cm}, 10 \times 40 \mathrm{~cm}$, $15 \mathrm{~cm} \times 2 \mathrm{~m}$ ( $\triangleright$ Abb. 2.24).

Nicht gelistet

- Adaptic (SYS)

- Bactigras (S\&N)

- Branolind (HAR)

- Cuticell/ Cuticell Classic (BSN)

- Lomatuell H (L\&R)

- Nobacutis (NOB)

- Tegaderm Contact (3M).

\section{Distanzgitter mit Silber}

Gelistet in den Helios-Kliniken

- Acticoat Flex $3(S \& N) 5 \times 5 \mathrm{~cm}, 10 \times 10 \mathrm{~cm}, 10 \times 20 \mathrm{~cm}, 20 \times 40 \mathrm{~cm}$ ( $\triangleright$ Abb. 2.25)

- Atrauman Ag (HAR) $5 \times 5 \mathrm{~cm}, 10 \times 10 \mathrm{~cm}, 10 \times 20 \mathrm{~cm}(\triangleright$ Abb. 2.26 , Abb. 2.27).

Vergleichbare Produkte

- Acticoat Flex 7 (S\&N)

- Physiotulle Ag (COL)

- UrgoTül Silver, Urgotül S.Ag (URG). 


\section{Distanzgitter mit sonstigen Antiseptika ( $\mathrm{Abb} .2 .83$ )}

Gelistet in den Helios-Kliniken

- Actilite (ADV) mit Manuka-Honig $5 \times 5 \mathrm{~cm}, 10 \times 10 \mathrm{~cm}, 20 \times 30 \mathrm{~cm}$

Vergleichbare Produkte

- Bactigras (S\&N) mit Chlorhexidin

- Betaisodona Wundgaze (MUN) mit Jod-PVP

- Medihoney (MDH) antibakterieller Tüllverband mit Manuka-Honig

- Oleo-Tüll classics (SAN), Sofra-Tüll classics (SAN) beide mit Honig.

\section{Distanzgitter hydroaktiv beschichtet}

Gelistet in den Helios-Kliniken

Keine.

\section{Vergleichbare Produkte}

- Hydrotüll (HAR) mit hydroaktiver Salbenmasse

- Intrasite Conformable (S\&N) mit Hydrogel

- Lomatuell Pro (L\&R) mit Vaseline und Hydrokolloid

- Physiotulle (COL) mit Hydrokolloid

- Urgotül (URG) mit Lipidokolloid

- Urgotül Duo (URG) mit TLC Wundheilungsmatrix.

\section{Distanzgittter mit Silikonbeschichtung}

Gelistet in den Helios-Kliniken

Mepitel One (MÖL) $5 \times 7,5 \mathrm{~cm}, 7,5 \times 10 \mathrm{~cm}, 10 \times 18 \mathrm{~cm}, 17 \times 25 \mathrm{~cm}$

$(\triangleright$ Abb. 2.28).

Nicht gelistet

- Adaptic Touch (KCl)

- aktivmed Wunddistanzgitter sensitiv (AKT)

- Askina Silnet (BBR)

- Atrauman Silicone (HAR)

- Dracotüll Silikon (DRA). 


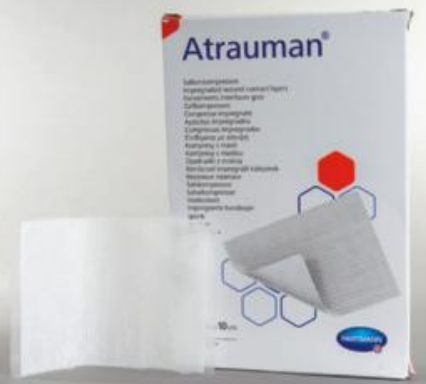

Abb. 2.21 Atrauman.

Abb. 2.22 Cuticerin.

(Mit freundlicher Genehmigung der Smith \& Nephew $\mathrm{GmbH}$ )

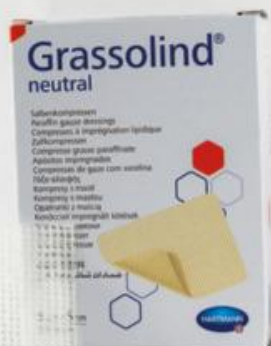

Abb. 2.23 Grassolind neutral.

Abb. 2.24 Jelonet.

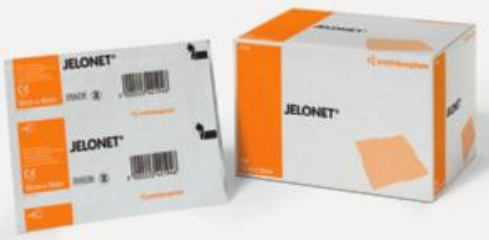

(Mit freundlicher Genehmigung der Smith \& Nephew $\mathrm{GmbH}$ ) 


\subsection{Standardverbandmaterial}

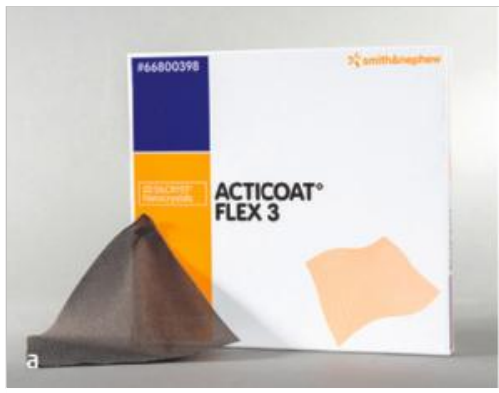

b

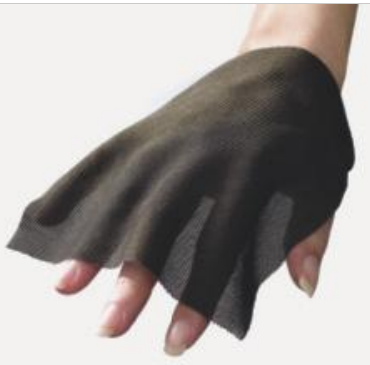

Abb. 2.25 Acticoat Flex 3. (Quelle: Mit freundlicher Genehmigung der Smith \& Nephew $\mathrm{GmbH})$

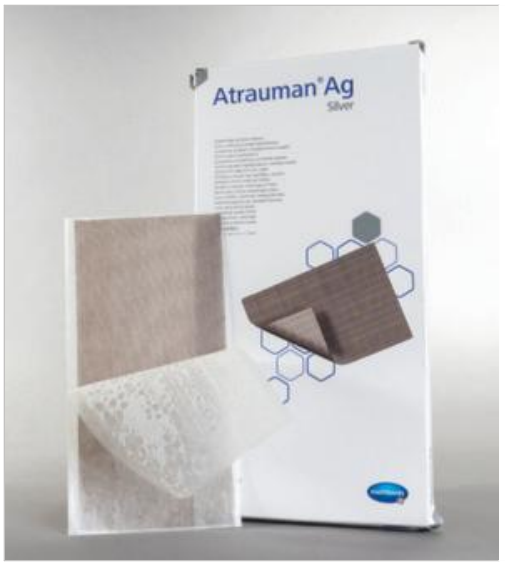

Abb. 2.26 Atrauman Ag.

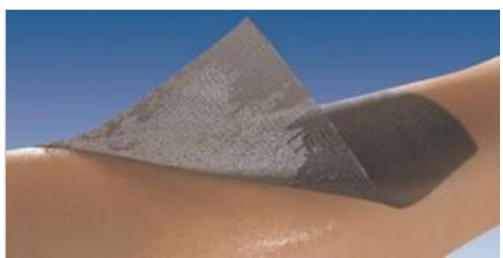

Abb. 2.27 Atrauman Ag: Anwendung. (Quelle: Mit freundlicher Genehmigung der Paul Hartmann AG) 


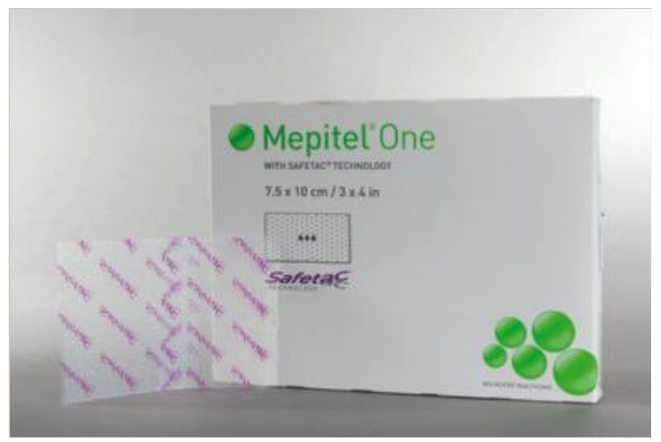

Abb. 2.28 Mepitel One.

\section{Beschreibung}

Einfache Wunddistanzgitter (Gazen) erlauben mit ihrer Gitterstruktur aus Baumwollgewebe, Zellulose oder Kunstfasern etc. einen Exsudatabfluss unter Feuchthaltung der Wunde. Gleichzeitig verhindern sie ein Verkleben mit den darüber aufgebrachten Saugkompressen. Dies geschieht durch Imprägnierung mit beispielsweise pflanzlichen Fettsäuren, Vaseline, dickflüssigen Paraffinen oder auch Öl-in-Wasser-Emulsionen.

\section{Merke}

Die Exsudatdurchlässigkeit ist auch von der Maschengröße abhängig.

Neben den einfachen Distanzgittern gibt es zahlreiche Modifikationen mit Silberzusatz, hydroaktiven oder Silikon-Beschichtungen für besondere Einsatzbereiche. $\mathrm{Zu}$ beachten sind die erheblichen Preisunterschiede!

Das bei Helios gelistete Produkt Acticoat flex 3 ist vom Aufbau her ein Distanzgitter mit extrem hoher Silberfreisetzung. Es unterscheidet sich dadurch wesentlich von anderen mit Silber beschichteten Gittern. Es ist antiseptisch sehr wirksam, aber aufgrund der hohen Silberfreisetzung für einen langfristigen Einsatz nicht geeignet. 


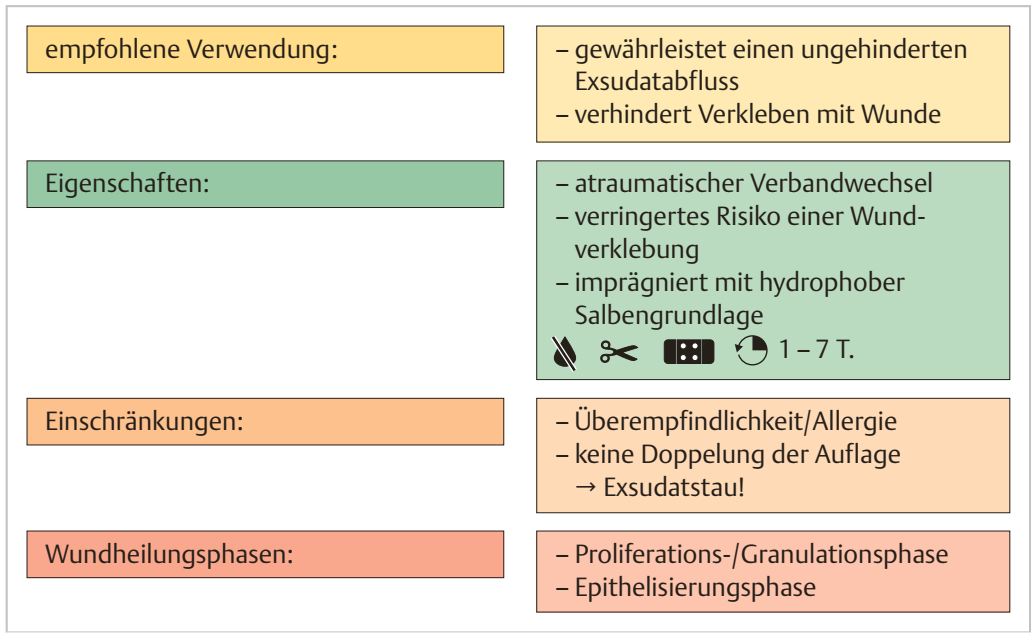

Abb. 2.29 Distanzgitter (Gaze).

\section{Indikation (Beispiele)}

- Schürf- und Risswunden

- Verbrennungen ersten und zweiten Grades

- Meshgraft, Hauttransplantate

- Spalthautentnahmestellen

- mäßig bis stark exsudierende oberflächliche Wunden

- exulzerierende Tumorwunden

- VAC-versorgte Wunden (silikonbeschichtete Wunddistanzgitter).

\section{Kontraindikation}

Überempfindlichkeit/Allergie gegen Bestandteile der Wundauflage. 


\section{Anwendungsweise}

- Distanzgitter können geschnitten werden. Sie sollen einen atraumatischen bzw. schmerzfreien Verbandwechsel gewährleisten. Vor Aufbringen wird die Wunde gereinigt und desinfiziert. Das Distanzgitter kann über den Wundrand hinausgelegt werden. Es soll plan platziert werden.

- Bei stark exsudierenden Wunden empfiehlt sich zusätzlich eine saugfähige Wundabdeckung, bei nachlassendem Exsudat dient eine nicht mit der Wunde verklebende Auflage oder ein semipermeabler Folienverband zur Fixierung.

- Das Entfernen der Wunddistanzgitter, insbesondere der mäßig gefetteten grobmaschigen Gazen, wird durch Spülen oder vorherigem Anfeuchten mit Ringerlösung erleichtert.

\section{Cave}

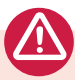

Nicht mehrere Lagen übereinanderlegen, da sonst unter dem dichten Abschluss eine feuchte Kammer entsteht! (Der Querschnitt der Löcher wird dadurch verringert.)

\section{Sekundärabdeckung:}

- Auf das Wunddistanzgitter wird eine sterile Kompresse o. Ä. aufgebracht.

- Die Fixierung kann mit einem Pflaster, einer Binde oder Folie erfolgen.

\section{Verbandwechsel:}

- Je nach Art der Wunde und des verwendeten Wunddistanzgitters sollte der Verbandwechsel im Intervall von 1-7 Tagen erfolgen.

- Bei silikonbeschichteten Gittern kann das Wechselintervall deutlich größer sein. Das ist jedoch eher eine Ausnahme. 


\subsection{Alginate}

\subsubsection{Alginate ohne antiseptische Wirkung}

\section{Produkte}

\section{Gelistet in den Helios-Kliniken}

Biatain Alginate (COL) $5 \times 5 \mathrm{~cm}, 10 \times 10 \mathrm{~cm}, 15 \times 15 \mathrm{~cm}$ ( $\vee$ Abb. 2.30).

Vergleichbare Produkte

- Algisite M (S\&N)

- Askina Sorb (BBR)

- Cutimed Alginate (BSN)

- DracoAlgin (DRA)

- Kaltostat (CON)

- Melgisorb Plus (MÖL)

- Sorbalgon (HAR)

- Suprasorb A (L\&R)

- Tegaderm Alginate (3M)

- Trionic (SYS).

Abb. 2.30 Biatain Alginate.

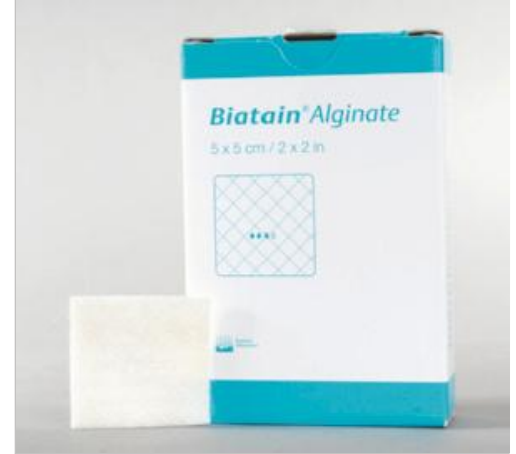

\section{Abb. 2.30 Biatain Alginate.}

\section{Biatain' Alginate}




\section{Beschreibung}

Alginate stellen einen lockeren Verband aus Kalziumalginatfasern dar, dadurch lassen sich die Kompressen auch zum Tamponieren verwenden und gut in tiefe und zerklüftete Wunden einbringen. Alginat wird aus Braunalgen gewonnen, es handelt sich um ein Polysaccharid aus Mannuronsäure und Guluronsäure $(\triangleright$ Abb. 2.31).

Kalziumalginatfasern wandeln sich im Kontakt mit Natriumsalzen, die im Blut und Wundexsudat vorhanden sind, unter Volumenzunahme in 2-3 Tagen in ein feuchtes Hydrogel von schleimiger Konsistenz um. Dabei werden Keime und Zelltrümmer in die Gelstruktur eingeschlossen. Das Ausmaß und die Geschwindigkeit der Gelbildung sind von der absorbierten Exsudatmenge abhängig.

Übersicht s. \ Abb. 2.32 .

\section{Indikation}

- exsudierende Wunden

- einsehbare Wundhöhlen/-taschen

- geeignet für verschmutzte und ggf. kolonisierte Wunden in Kombination mit Silber (bei schwach exsudierenden Wunden mit Ringerlösung, ggf. wässriger Antiseptikalösung tränken)

- geeignet zur Behandlung von leicht blutenden Wunden, einschließlich Schnitt- und Risswunden (hämostyptische Eigenschaften).

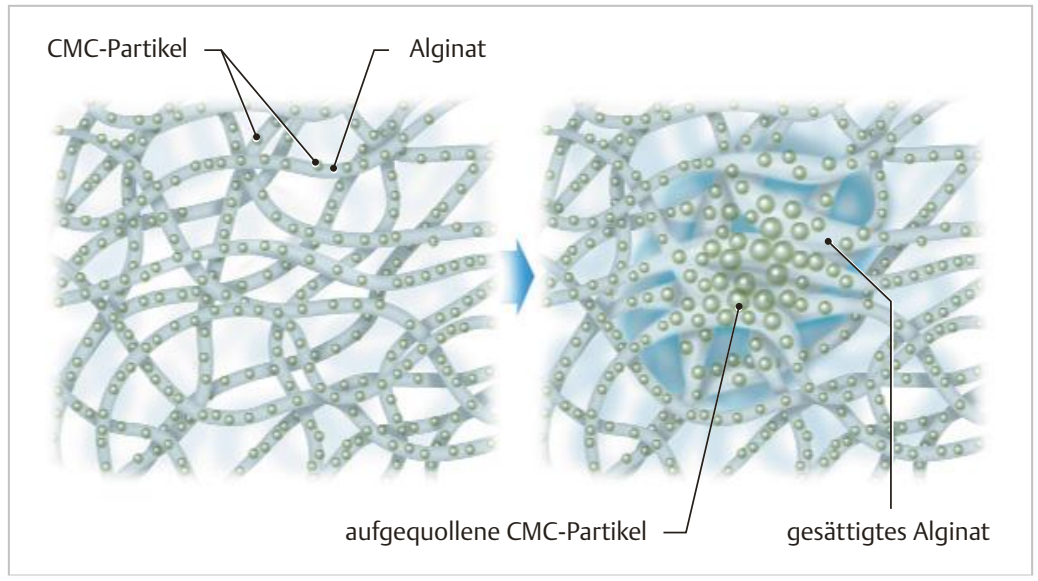

Abb. 2.31 Biatain Alginate: Schema. 


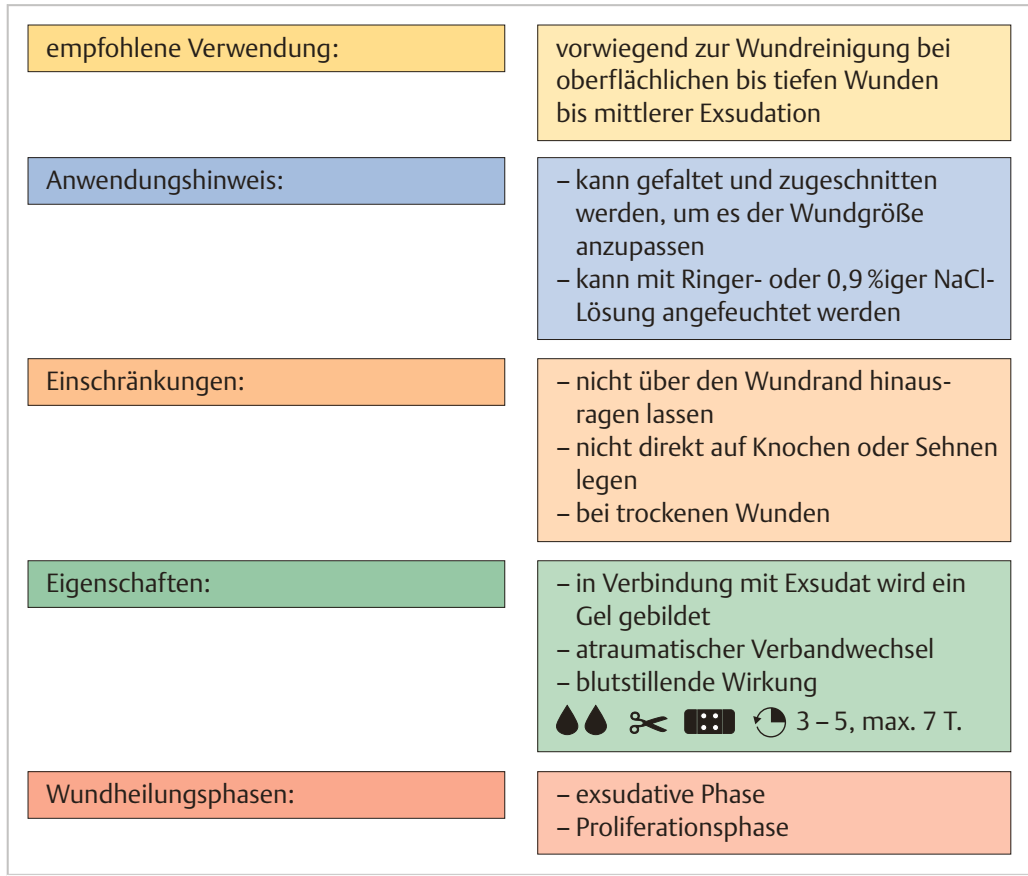

Abb. 2.32 Alginate ohne antiseptische Wirkung.

\section{Kontraindikationen}

Trockene Wunden:

- Gefahr der Austrocknung von schwach exsudierenden Wunden mit Bildung einer Oberflächennekrose

- Bei Verwendung an Sehnen, Knochen oder Knorpelgewebe besteht die Gefahr der Austrocknung und damit der Zerstörung der Zellschichten von Peritendineum, Periost oder Perikartilagineum - Schutz z. B. mit Hydrogelen.

Nicht in tiefe, nicht einsehbare Wundhöhlen oder Fistelgänge einbringen.

Cave: Verbleib des Materials! 


\section{Anwendungsweise}

- Alginat kann gefaltet, gezupft oder geschnitten werden

- bei schwach bis mäßig exsudierenden Wunden $\rightarrow$ Anfeuchtung (s. o.)

- bei tiefen Wunden oder Wundtaschen das Alginat mehrfach falten, auslegen und locker tamponieren (nur zu zwei Dritteln auffüllen!)

- Kalziumalginat darf nicht die Wundumgebung bedecken, da es sonst zu ausgeprägter Mazeration kommen kann (horizontaler Exsudattransport)

- auch unter einem Kompressionsverband einsetzbar

\section{Merke}

Zuschneiden auf Wundgröße, nicht über den Wundrand legen, da Mazerationsgefahr.

\section{Sekundärabdeckung:}

- bei geringer Exsudation: Folie

- bei mäßiger Exsudation: sterile Kompressen/Saugkompressen und Folie

- bei stärkerer Exsudation: Schaumverband (mit Superabsorber) oder Superabsorber (Kissen).

\section{Cave}

Vergelendes Alginat kann die Poren der Sekundärabdeckung verstopfen und zur Exsudatretention führen, Mazeration am Wundrand beachten!

\section{Verbandwechsel:}

- Der Verband wird je nach anfallendem Exsudat im Abstand von 3-5 Tagen erneuert (maximal nach sieben Tagen).

- Exsudations-/Reinigungsphase: alle 1-3 Tage.

- Granulations-/Proliferationsphase: bis zu 7 Tage.

- Gelrückstände oder am Wundrand festklebende Alginatfasern lassen sich mit Ringerlösung abwaschen. Dennoch in der Wunde verbleibende Fasern sind biologisch abbaubar und unbedenklich. 


\subsubsection{Alginate mit antiseptischer Wirkung}

\section{Produkte}

\section{Gelistet in den Helios-Kliniken}

- Biatain Alginate $\mathrm{Ag}(\mathrm{COL}) 5 \times 5 \mathrm{~cm}, 10 \times 10 \mathrm{~cm}, 15 \times 15 \mathrm{~cm}$ ( $\vee$ Abb. 2.33).

\section{Vergleichbare Produkte}

- Algisite AG (S\&N)

- Melgisorb AG (MÖL)

- Silvercel (SYS)

- Suprasorb A + Ag (L\&R)

- Tegaderm Alginate Ag (3M).

\section{Beschreibung}

Bei Kontakt mit Wundexsudat wird Biatain Alginate Ag zu einem weichen, kohäsiven $\mathrm{Gel}$, das sich den Konturen des Wundgrundes anpasst und so für Infektionsvorbeugung sorgt ( $\vee$ Abb. 2.34). Der Silberkomplex setzt bei Anwesenheit von Wundexsudat Ionen frei.

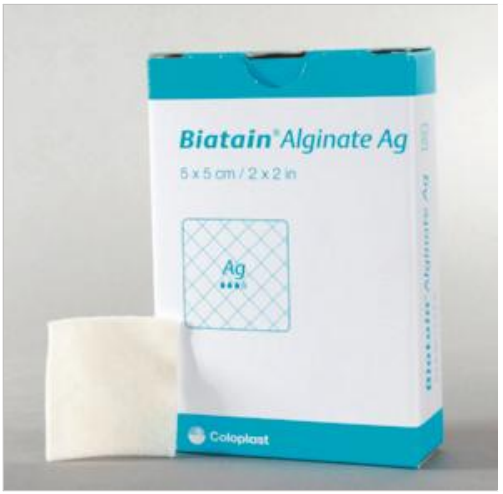

Abb. 2.33 Biatain Alginate Ag. 


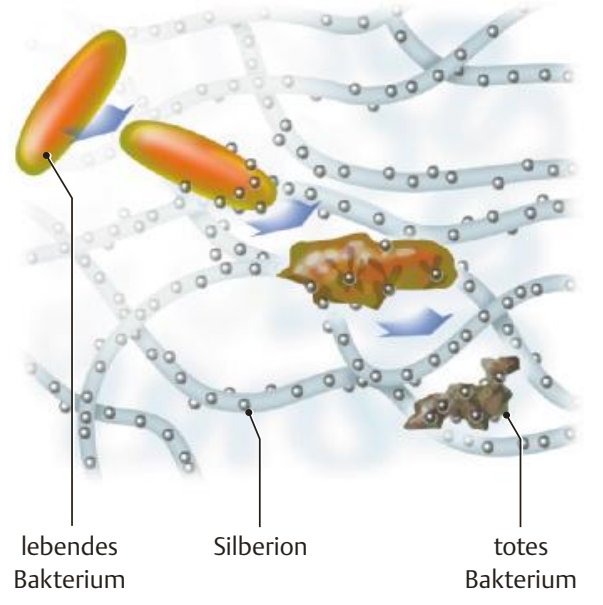

Abb. 2.34 Biatain Alginate

Ag: Schema.

\section{Empfohlene Verwendung}

- Mittelstark bis stark exsudierende Wunden, stärker kolonisiert oder infiziert (breite antimikrobielle Wirksamkeit auch gegen Candida albicans, MRSA, ESBL und VRE).

- Biatain Alginate Ag kann bei Wunden mit Infektionsrisiko eingesetzt werden; auch unter einem Kompressionsverband einsetzbar.

\section{Kontraindikation}

Silberunverträglichkeit.

Übrige Beurteilung analog zu Kap. 2.3.1. 


\subsection{Hydrofaserverbände}

\subsubsection{Hydrofaser ohne antiseptische Wirkung}

\section{Produkte}

\section{Gelistet in den Helios-Kliniken}

- Aquacel Extra Wundauflage (CON) $5 \times 5 \mathrm{~cm}, 10 \times 10 \mathrm{~cm}, 15 \times 15 \mathrm{~cm}$ ( $\vee$ Abb. 2.35)

- Aquacel Tamponade mit Verstärkungsfasern (CON) $2 \times 45 \mathrm{~cm}$.

\section{Vergleichbare Produkte}

- Biosorb (SYS)

- Durafiber (S\&N)

- Exufiber (MÖL)

- Suprasorb Liquacel (L\&R).

\section{Beschreibung}

Die Hydrofaser oder Hydrofiber besteht aus Natriumcarboxymethylcellulose und ist erhältlich als Kompresse oder Tamponade. Die Auflage ist weich, gut drapierbar und wandelt sich bei Kontakt mit Exsudat in ein transparentes, weiches, formstabiles Gel um, das nicht mit der Umgebung verklebt.

Die enthaltenen Vliese oder Granulate können große Flüssigkeitsmengen aufnehmen (bis zum Vielfachen des eigenen Volumens und Trockengewichts), bleiben dabei formstabile Gele („Pampersprinzip“).

Die fehlende horizontale Ausbreitung der Flüssigkeit in Hydrofaserverbänden verhindert eine Mazeration des Wundrandes.

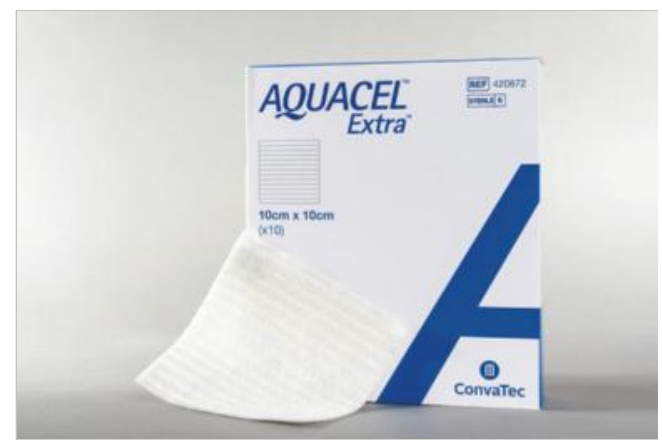

Abb. 2.35 Aquacel Extra. 
Zelltrümmer und Wundexsudat werden zwischen den Fasern aufgenommen und fest eingebunden. Die feste Einbindung bleibt solange stabil, bis die Faser übersättigt ist. Die Hydrofasern tragen dadurch auch zur Wundreinigung bei und fördern zudem die Granulationsbildung.

Hydrofaserverbände sind innerhalb von Sekunden vollgesogen, damit ist die Gelbildung mehr als 10 000-fach schneller als bei Alginaten. Dies kann ebenso wie Alginaten eine schwach exsudative Wunde trockenlegen mit der Gefahr der Nekrosebildung.

Übersicht s. \ Abb. 2.36 .

empfohlene Verwendung:

Anwendungshinweis:

Einschränkungen:

Eigenschaften:

Wundheilungsphasen: vorwiegend zur Wundreinigung (Lösen von Fibrinbelägen) bei oberflächlichen bis tiefen Wunden und mäßiger bis starker Exsudation

- kann gefaltet und zugeschnitten werden

- darf über den Wundrand hinaus gelegt werden!

- kann mit Ringer- oder 0,9\%iger $\mathrm{NaCl}$-Lösung angefeuchtet werden

- trockene Wunden

- Cave: nicht direkt auf Knochen oder Sehnen legen!

- in Verbindung mit Exsudat wird ein Gel gebildet

- Durch die Verstärkungsfasern ist ein rückstandsfreies Entfernen aus der Wunde möglich.

- atraumatischer Verbandwechsel

- verringert die Mazeration in der Wundumgebung

- Typisch ist die vertikale Saugrichtung mit Beschränkung auf die unmittelbare Wunde.

000 \&:: $33-5, \max .7 \mathrm{~T}$.

- exsudative Phase

- Proliferationsphase

Abb. 2.36 Hydrofaser ohne antiseptische Wirkung. 


\section{Indikationen}

- exsudierende Wunden

- Wundhöhlen/-taschen

- geeignet für verschmutzte und ggf. kolonisierte Wunden in Kombination mit Silber.

\section{Kontraindikationen}

- Gefahr der Austrocknung von schwach exsudierenden Wunden mit Bildung einer Oberflächennekrose.

- Bei Verwendung an Sehnen, Knochen oder Knorpelgewebe besteht die Gefahr der Austrocknung und damit der Zerstörung der Zellschichten von Peritendineum, Periost oder Perikartilagineum $\rightarrow$ Schutz mit Hydrogel.

\section{Anwendungsweise}

- Hydrofaser kann gefaltet oder geschnitten werden.

- Bei mäßig bis stark exsudierenden Wunden lässt sich die Kompresse/Tamponade ohne Befeuchtung benutzen.

- Der Verband ist so zu applizieren, dass er die Wundränder ca. 1-2 cm überlappt.

- Hydrofaser ist bei Mazeration des Wundrandes und der Umgebungshaut verwendbar.

\section{Sekundärabdeckung:}

- bei geringer Exsudation: Folie

- bei mäßiger Exsudation: sterile Kompressen/Saugkompressen und Folie

- bei stärkerer Exsudation: Schaumverband oder Superabsorber.

\section{Verbandwechsel:}

- Der Verband wird je nach anfallendem Exsudat im Abstand von 3-5 Tagen erneuert (maximal nach 7 Tagen).

- Exsudations-/Reinigungsphase: alle 1-3 Tage

- Granulations-/Proliferationsphase: bis zu 7 Tage. 


\subsubsection{Hydrofaser mit antiseptischer Wirkung (Silber)}

\section{Produkte}

\section{Gelistet in den Helios-Kliniken}

- Aquacel extra Ag Wundauflage (CON) $5 \times 5 \mathrm{~cm}, 10 \times 10 \mathrm{~cm}, 15 \times 15 \mathrm{~cm}$ ( $\triangleright$ Abb. 2.37)

- Aquacel extra Ag Tamponade mit Verstärkungsfasern (CON) $2 \times 45 \mathrm{~cm}$ ( $\triangleright$ Abb. 2.37).

\section{Vergleichbare Produkte}

- Durafiber Ag (S\&N)

- Textus bioactiv (BIC)

- Textus balance (BIC).

\section{Beschreibung}

Die Silberionen inaktivieren vorhandene Mikroorganismen im gelierten Verband.

Nach einer Wundreinigung mit einem flüssigen Antiseptikum (z.B. Lavasorb) muss vor der Applikation von Aquacel extra Ag die Wunde mit Ringeroder 0,9\%iger $\mathrm{NaCl}$-Lösung neutralisiert werden. Aquacel extra Ag benötigt ausreichend Feuchtigkeit, damit die Silberionen ihre antimikrobielle Wirkung im Verband entfalten können.

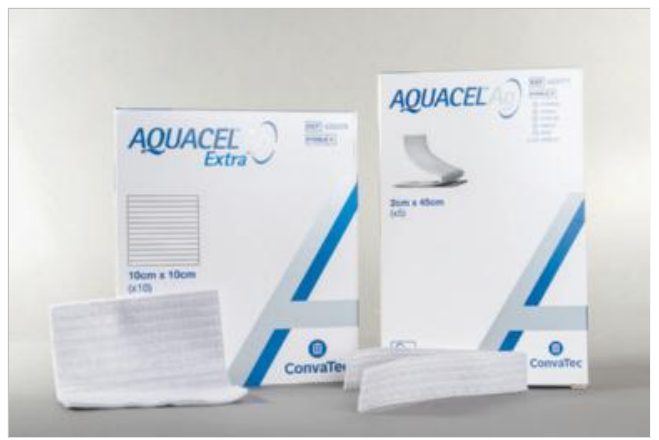

Abb. 2.37 Aquacel Extra Ag: Wundauflage und Tamponade. 


\section{Empfohlene Verwendung}

Stärker kolonisierte oder infizierte Wunden (breite antimikrobielle Wirksamkeit auch gegen Candida albicans, MRSA, ESBL und VRE).

Übrige Beurteilung analog zu Kap. 2.4.1.

\section{Zusätzliche Kontraindikationen}

- keine Verwendung mit Produkten auf Öl-/Paraffin-/Vaselinebasis

- Silberunverträglichkeit.

\subsubsection{Unterschied zwischen Alginat und Hydrofaser}

Die Indikationen für deren Einsatz sind vergleichbar.

\section{Merke}

Hydrofaser quillt in die „Höhe“, Alginat läuft in die „Breite“.

\section{Alginat:}

- organischer Stoff aus Braunalgen

- Aufnahme von Wundexsudat erfolgt horizontal, ähnlich wie bei Löschpapier

- muss auf die Wundgröße zugeschnitten/gezupft werden, da ansonsten die Gefahr einer Mazeration des Wundrandes/der Umgebungshaut besteht

- erst nach Ionenaustausch entsteht ein hydrophiles Gel

- bindet allmählich Exsudat, nimmt gelöste Bestandteile aus der Wunde auf und schließt diese fest ein.

\section{Hydrofaser:}

- aus Natriumcarboxymethylcellulose

- Aufnahme von Wundexsudat erfolgt vertikal

- Schutz des Wundrandes vor Mazeration

- bindet schlagartig Exsudat, nimmt gelöste Bestandteile aus der Wunde auf und schließt diese fest ein. 


\subsection{Hydrogele}

\subsubsection{Beschreibung}

Hydrogele (Übersicht s. \ Abb. 2.38) bestehen aus einem dreidimensionalen Netzwerk hydrophyler Polyurethanpolymere in wässriger Lösung. Weiterhin sind je nach Anbieter Gelatine, Pektin, Natriumcarboxymethylcellulose und Propylenglykol beigefügt. Durch ihren Wasseranteil rehydrieren Hydrogele trockene Nekrosen und Beläge im Rahmen des autolytischen Débridements, auch Fibrinbeläge werden dabei gelöst. Hydrogele werden vorrangig zum schonenden Débridement eingesetzt, wenn keine chirurgische Intervention erfolgt. Aufgrund des hohen Wasseranteils werden Hydrogele vor allem in der Granulationsphase zum Feuchthalten eingesetzt.

Gele können sowohl Feuchtigkeit an die Wunde abgeben als auch überschüssiges Wundexsudat aufnehmen. Sie werden als Gel in der Tube bzw. Balgflasche zum Einbringen in tiefere Wunden angeboten. Außerdem gibt es durchsichtige Gelkompressen, bestehend aus einer semipermeablen Folie, auf die ein Gel (z. B. Polyacrylamid-Agar-Gel) mit einem hohen Wasseranteil aufgebracht ist.

empfohlene Verwendung:

Anwendungshinweis:

Einschränkungen:

Eigenschaften:

Wundheilungsphasen: autolytisches Debridement

- Tube: 2 - 5 mm dick auftragen und semipermeabel abdecken

- Platte: auch unter Kompression anwendbar

- Anaerobierinfektionen

- infizierte, stark exsudierende oder blutende Wunden

- Feuchthaltung bei trockenen Wunden - Erweichung von trockenen Nekrosen

I:: 2-3, max. $3 \mathrm{~T}$.

- Debridement in der Exsudationsphase - Feuchthaltung in der Granulationsund Epithelisierungsphase

Abb. 2.38 Hydrogele. 
Manche Gele enthalten Propylenglykol (Gefahr einer seltenen allergischen Reaktion).

Gele sind semitransparent und unterschiedlich in der Konsistenz. Sie können, bezogen auf ihr Eigengewicht, nur bis zur doppelten Menge Wasser aufnehmen.

\subsubsection{Hydrogele in Tube oder Flasche}

\section{Produkte}

\section{Gelistet in den Helios-Kliniken}

Varihesive Hydrogel (CON) $15 \mathrm{~g}$ ( $\triangleright$ Abb. 2.39).

\section{Vergleichbare Produkte}

- Askina Gel (BBR)

- Cutimed Gel (BSN)

- Draco Hydrogel (DRA)

- Flaminal Hydro (FLE), Flaminal Forte (FLE)

- Hydrosorb Gel (HAR)

- IntraSite Gel (S\&N)

- Kendall amorphes Hydrogel (COV)

- NU-GEL (SYS)

- Purilon Gel (COV)

- Suprasorb G (L\&R)

- Tegaderm Hydrogel (3M)

- Urgo Hydrogel (URG).

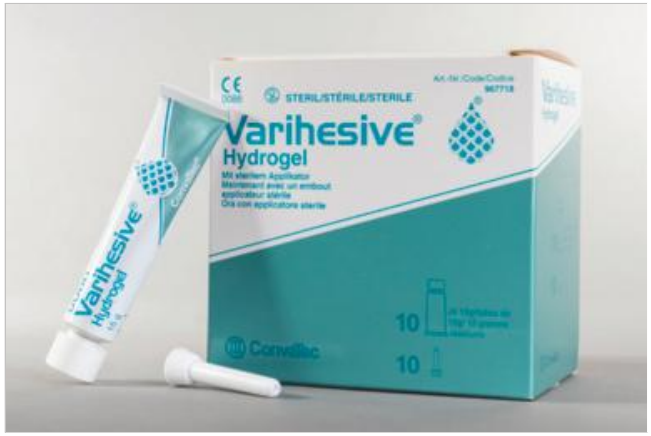

Abb. 2.39 Varihesive Hydrogel. 


\section{Indikation}

- zur Erweichung und damit Auflösung von trockenen Nekrosen

- zur Feuchthaltung bzw. Rehydration trockener Wunden und freiliegender Strukturen (Sehnen, Knochen)

- Verband nach Dermabrasion, Laseranwendung oder Chemical Peeling.

\section{Kontraindikationen}

- Verdacht auf Anaerobierinfektionen oder infizierte Wunden

- stark exsudierende oder blutende Wunden

- bei trockenen Nekrosen (pAVK) werden Hydrogele in der Regel nicht angewandt, da die allmähliche Ablösung erreicht werden soll.

\section{Cave}

Bei akralen Nekrosen kann unter Hydrogeltherapie eine feuchte Gangrän entstehen!

\section{Anwendungsweise}

Das Gel wird 2-5 mm dick aufgetragen oder in tiefe Wunden eingebracht.

Sekundärabdeckung: mit einer semipermeablen Wundauflage.

Verbandwechsel: Das Gel kann 2-3 Tage auf der Wunde verbleiben, Reste mit Ringerlösung entfernen.

\section{Merke}

Nicht konservierte Gele sind für den einmaligen Gebrauch bestimmt - Reste müssen verworfen werden. 


\subsubsection{Hydrogelplatten}

\section{Produkte}

\section{Gelistet in den Helios-Kliniken}

Hydrosorb (HAR) $10 \times 10 \mathrm{~cm}$ ( $\triangleright$ Abb. 2.40).

\section{Vergleichbare Produkte}

- Cutimed HydroControl (BSN)

- Hydrosorb Comfort mit Kleberand (HAR)

- Kendall Hydrogel Wundverband (COV)

- Nobagel-Verband (NOB)

- Suprasorb-G-Gel-Kompresse (L\&R).

\section{Empfohlene Verwendung}

- zur feuchten Wundbehandlung in der Granulations- und Epithelisierungsphase bei klinisch nicht infizierten oberflächlichen Wunden

- zur Versorgung von Verbrennungen bis Grad 2a

- zur Förderung der Reepithelisierung von Spalthautentnahmestellen nach vorausgegangener adäquater Blutstillung (zweite Wahl).

\section{Kontraindikationen}

- Verdacht auf Anaerobierinfektionen oder infizierte Wunden

- stark exsudierende oder blutende Wunden

- nicht verwendbar bei infizierten Wunden und Brandwunden ab Grad 3.

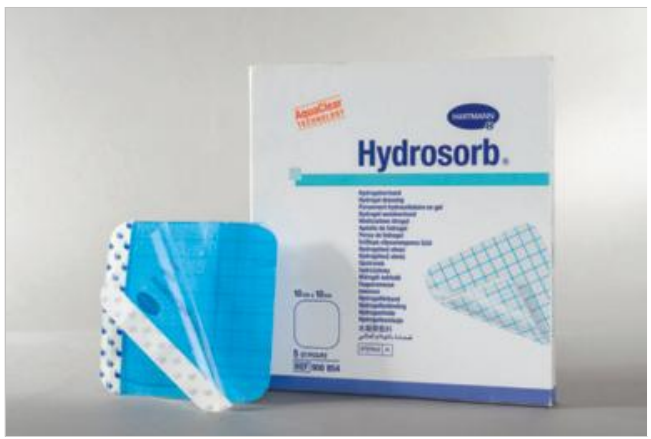

Abb. 2.40 Hydrosorb. 


\section{Anwendungshinweise}

- kann unter Kompression angewendet werden

- durch die beschreibbare Abziehfolie lässt sich die Wundgröße dokumentieren.

Sekundärabdeckung: Fixierpflaster oder Fixierbinde.

Verbandwechsel:

- Gelplatten können 1-7 Tage auf der Wunde verbleiben

- Wundbeurteilung durch Transparenz möglich!

\subsection{Hydrokolloide}

\section{Produkte}

\section{Gelistet in den Helios-Kliniken}

- Comfeel Plus flexibel (dicker) (COL) $4 \times 6 \mathrm{~cm} ; 10 \times 10 \mathrm{~cm} ; 15 \times 15 \mathrm{~cm}$ ( $\triangleright$ Abb. 2.41)

- Comfeel Plus Transparent (dünner) $(\mathrm{COL}) 5 \times 7 \mathrm{~cm}, 10 \times 10 \mathrm{~cm}, 15 \times 15 \mathrm{~cm}$, $5 \times 15 \mathrm{~cm}, 5 \times 25 \mathrm{~cm}(\triangleright$ Abb. 2.42).

\section{Vergleichbare Produkte}

- 3M Tegaderm Hydrokolloid, 3M Tegaderm Hydrokolloid thin (3M)

- Algoplaque, Algoplaque Film (URG)

- Askina Biofilm Transparent, Askina Hydro (BBR)

- Cutimed Hydro L, Cutimed Hydro B, Cutimed Hydro (BSN)

- DracoHydro / DracoHydro dünn (DRA)

- Hydrokoll, Hydrokoll thin (HAR)

- Kendall Hydrokolloid-Wundauflage (COV)

- Nobacolloid (NOB)

- Nu-Derm (SYS)

- Replicare (S\&N)

- ROGG Hydrokolloid (ROG)

- Suprasorb H (L\&R)

- Traumasive (HEX)

- Varihesive E, Varihesive E Border, Varihesive Extra dünn, Varihesive signal (CON). 


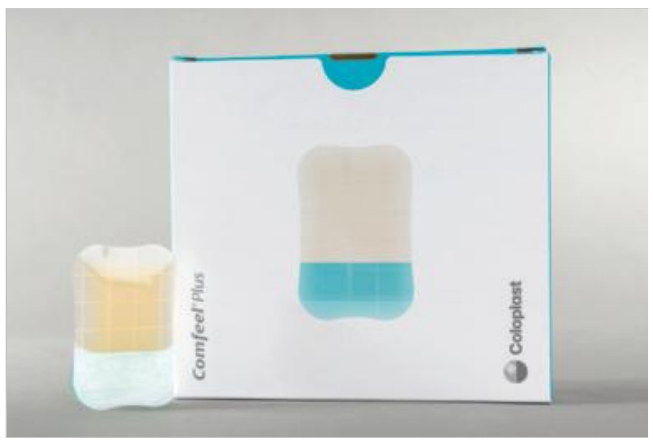

Abb. 2.41 Comfeel Plus flexibel.

\subsubsection{Beschreibung}

Abb. 2.42 Comfeel Plus transparent.

Selbstklebende Masse aus stark quellenden Partikeln, meistens Carboxymethylcellulose, Pektine oder Gelatine, in synthetischer Kautschukmasse eingebettet ( $\triangleright$ Abb. 2.43, $\triangleright$ Abb. 2.44).

Der Verband wird durch einen eingearbeiteten Polyurethanfilm abgedeckt. Diese Schicht ist semipermeabel, keim- und wasserdicht $\rightarrow$ Duschen ist möglich.

Dicke Hydrokolloide nehmen Exsudat auf, sind aber weniger flexibel (nicht in Gelenknähe benutzen).

Dünne Hydrokolloide nehmen wenig Exsudat auf, passen sich besser an Hautkonturen an.

Übersicht s. > Abb. 2.45. 


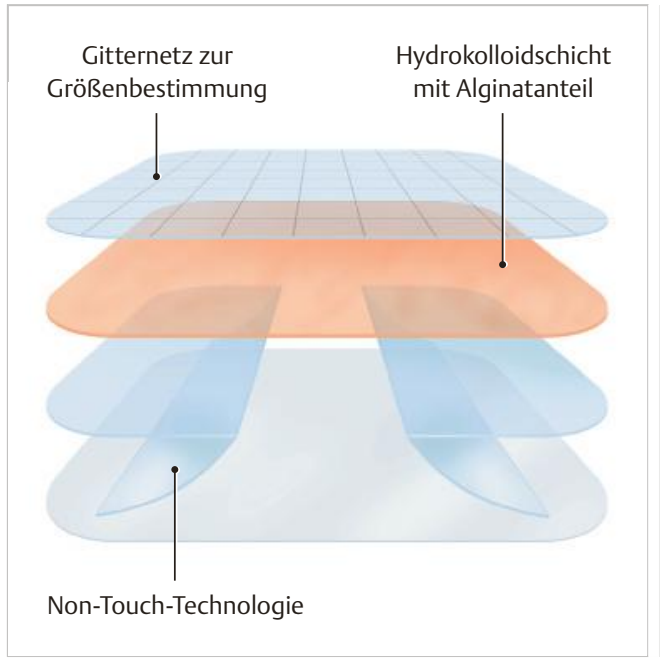

Abb. 2.43 Comfeel Plus transparent: Aufbau.

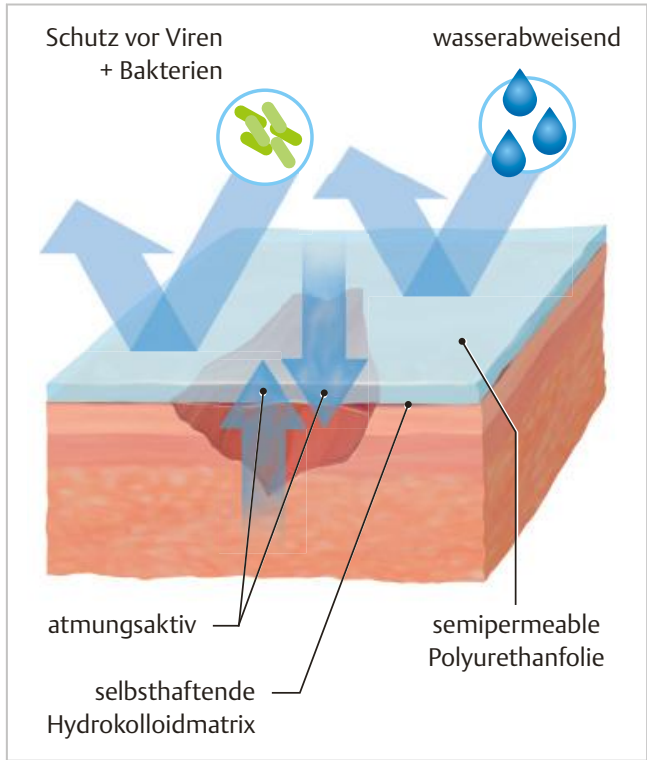

Abb. 2.44 Comfeel Plus transparent: Funktion. 


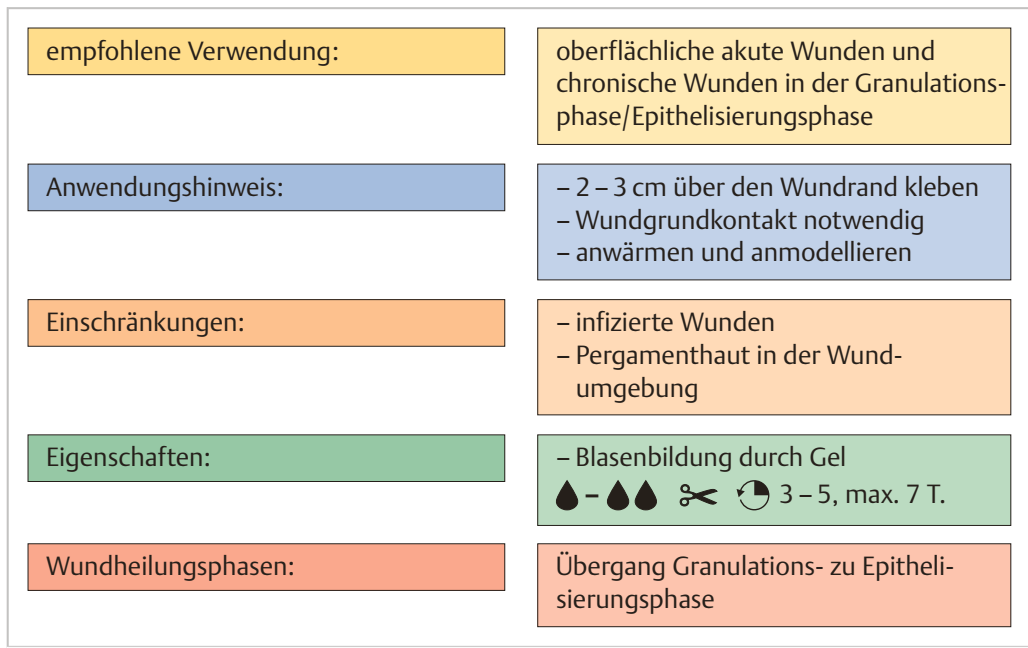

Abb. 2.45 Hydrokolloide.

\subsubsection{Indikation}

- wenig bis mäßige exsudierende Wunden (je nach Schichtdicke)

- oberflächliche Wunden

- infektionsfreie Wunden

- Spalthautentnahmestellen (zweite Wahl)

- Sekundärverband (zweite Wahl)

- dünnere Platten können als Hautschutz, auch in Wundumgebung, verwendet werden (Katheter, Dekubitus etc.).

\section{Kontraindikationen}

- infizierte Wunden; aufgrund der relativen Hypoxie unter dem Verband

- Verbrennungen > Grad 2a

- ischämische Ulzera

- direkter Kontakt zu freiliegenden Knochen oder Sehnen

- Tumorwunden

- Pergamenthaut. 


\subsubsection{Anwendungsweise}

- Verband ist undurchlässig von außen gegen Wasser, Schmutz und Keime.

- Feuchthalten der Wunde bei gleichzeitiger Verdunstung von Wasserdampf (semiokklusiv).

- Durch Exsudataufnahme entsteht eine an Eiter erinnernde Gel-Blase im Verband. Das Gel nimmt dabei eine gelbliche, grünliche oder bräunliche Färbung an und hat einen unangenehmen süßlichen Geruch $\rightarrow$ nicht mit Eiter verwechseln!

- Beim Verbandwechsel muss diese Gelschicht entfernt werden.

- Bei Pergamenthaut Gefahr des „Tape-Strippings“ (= Entfernung der obersten Hautschicht).

- Bei stark behaarter Haut die Haare vor der Applikation entfernen.

- Faltenfreie Aufbringung.

- Die Wundauflage mit der Hand körperwarm anmodellieren $\rightarrow$ optimale Haftung.

- Hydrokolloid ca. 2-3 cm über den Wundrand hinaus kleben.

- Bei großen Wunden oder an Gelenken können mehrere Verbände dachziegelartig geklebt werden.

- Wundgrundkontakt ist sicherzustellen oder ggf. mit Wundfüller auszugleichen.

- Sonderformen für Ellenbogen, Fersen und Sakralbereich sind vorhanden, alternativ Verwendung dünner Hydrokolloide durch Zuschneiden und Dachziegeltechnik.

Anwendungbeispiele s. > Abb. 2.46.

\section{Sekundärabdeckung:}

- nicht notwendig

- an stark belasteten Stellen Folienverband zur Fixierung möglich.

\section{Cave}

Zentralen Bereich zur Wasserdampfabdunstung freilassen!

\section{Verbandwechsel:}

- nach 3-7 Tagen

- überschreitet die Blase den Umfang der Wunde $\rightarrow$ Verbandwechsel

- Verband schonend lösen, z. B. überdehnen

- Beurteilung der Wundverhältnisse erst nach Wundreinigung möglich. 

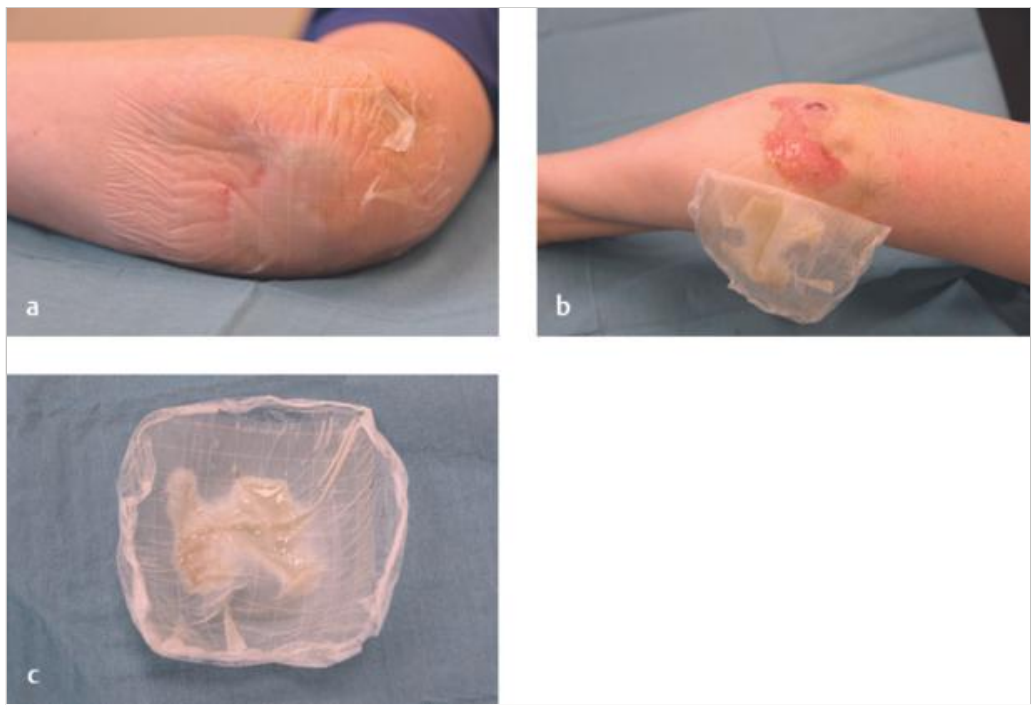

Abb. 2.46 Hydrokolloid: Anwendung.

a Hydrokolloid in der Anwendung mit zentraler kolloidaler Phasenumkehr (kein Eiter!).

b Partiell entferntes Hydrokolloid.

c Entferntes Hydrokolloid mit zentraler Phasenumkehr.

\subsection{Schaumstoffverbände (Hydropolymere)}

\subsubsection{Einführung}

Schaumstoffverbände sind Hydropolymere und bestehen meistens aus aufgeschäumtem Polyurethanschaum (PU-Schaum) unterschiedlicher Porengröße und Oberflächenbeschaffenheit (offenporig/geschlossenporig).

Offenporige Schäume fördern das Einsprossen von Kapillaren in den Schaum $\rightarrow$ großer Granulationsreiz. Daher entsteht beim Verbandwechsel eine frische, blutende Wunde. Neben dem Wundexsudat wird auch ein Teil des frischen Granulationsgewebes entfernt $\rightarrow$ Wundkonditionierung vor Transplantation.

Geschlossenporige Schäume werden durch Bedampfung der Oberfläche hergestellt und verhindern dadurch das Einsprossen von Kapillaren. In der modernen Wundversorgung werden fast ausschließlich letztere angewendet.

- Die Außenseite ist weitgehend mit semiokklusiver PU-Folie bestückt $\rightarrow$ Wäscheschutz. 
- Die Innenseite ist teilweise zusätzlich mit Silikon, Distanzgitter o. Ä. zur Wunde hin beschichtet.

- Schaumverbände können unter Kompression aufgenommene Flüssigkeit nicht zurückhalten, sondern pressen sie in die Wunde zurück. Abhilfe schafft die Einarbeitung von Superabsorbern (Polyacrylate) in den Schaumkörper.

- Schäume eignen sich als Primär- und Sekundärabdeckung.

Es gibt feinporige Schäume, die trotz hoher Aufnahmekapazität aufgrund ihrer Struktur kein dickflüssiges Exsudat aufnehmen können. Sie verkleben nicht mit der Wunde, der Verbandwechsel ist schmerzarm.

Grobporige Schäume sind besser für die Aufnahme von dickflüssigem Exsudat und Zelltrümmern geeignet. Sie unterstützen damit das autolytische Débridement. Allerdings kann Granulationsgewebe einwachsen und starke Schmerzen beim Verbandwechsel bereiten $\rightarrow$ Cave: Verbandwechsel nach weniger als 7 Tagen!

Übersicht s. \ Abb. 2.47.

empfohlene Verwendung:

Anwendungshinweis:

Eigenschaften von PolyurethanVerbänden:

Eigenschaften grobporiger Schaumverband:

Eigenschaften kleinporiger Schaumverband:

Eigenschaften Schaumstoff mit Superabsorber:

Einschränkungen:

Wundheilungsphasen: leicht bis stark exsudierende Wunden

Mazerationsgefahr bei Kompression, bei ausgeschöpfter Exsudationsaufnahmekapazität

- strukturbeständig

- quellen auf und füllen die Wunde auf

gute Aufnahme von dickflüssigem, zähem Exsudat

gute Aufnahme von dünnflüssigem Exsudat

Flüssigkeitsretention auch unter Kompression

- trockene nekrotische Wunden (pAVK)

- Cave: infizierte Wunde

- Exsudationsphase

- Proliferations-/Granulationsphase

Abb. 2.47 Schaumstoffverbände (Hydropolymerverbände). 


\section{Indikation}

Schwach bis stark exsudierende Wunden.

\section{Kontraindikationen}

- Auftreten von Wundrandmazerationen (horizontale Exsudatableitung)

- Infektionen (häufigere Verbandwechsel)

- Wunden bei pAVK IV.

\section{Anwendungsweise}

Verbandwechsel: Wechsel sobald der Verband erschöpft ist („der Rand der Wundauflage erreicht ist“), in der Regel alle 2-7 Tage.

\subsubsection{Hydropolymerverbände ohne Superabsorber, geschlossenporig}

\section{Produkte}

\section{Gelistet in den Helios-Kliniken}

mit mittlerer bis starker Exsudataufnahme

- Biatain Schaumverband

$\circ$ nicht haftend (COL) ( $\triangleright$ Abb. 2.48)

- selbsthaftend (COL) ( $\triangleright$ Abb. 2.49)

- sanft haftend (nicht gelistet) (COL)

- Biatain Cavity (COL)

- Biatain-Ibu (COL) (๖ Abb. 2.50)

- Biatain Ag Schaumverband (COL)

- Biatain Silicone (COL)

im Detail:

- Biatain Schaumverband nicht haftend $5 \times 7 \mathrm{~cm}, 10 \times 10 \mathrm{~cm}, 10 \times 20 \mathrm{~cm}$, $15 \times 15 \mathrm{~cm}, 20 \times 20 \mathrm{~cm}$

- Biatain Schaumverband selbsthaftend 7,5 × 7,5 cm, 12,5×12,5cm, $18 \times 18 \mathrm{~cm}$

- Biatain-lbu nicht haftend $10 \times 10 \mathrm{~cm}, 10 \times 20 \mathrm{~cm}, 20 \times 20 \mathrm{~cm}$

- Biatain Ag Schaumverband nicht haftend $5 \times 7 \mathrm{~cm}, 10 \times 10 \mathrm{~cm}, 10 \times 20 \mathrm{~cm}$, $15 \times 15 \mathrm{~cm}, 20 \times 20 \mathrm{~cm}$ ( $\triangleright$ Abb. 2.51) 
- Biatain Schaumverband Cavity $5 \times 8 \mathrm{~cm}$

- Biatain Ag Schaumverband selbsthaftend 7,5 ×7,5 cm, 12,5×12,5cm, $18 \times 18 \mathrm{~cm}(\triangleright$ Abb. 2.52)

- Biatain Ag Schaumverband Cavity $5 \times 8 \mathrm{~cm}$ ( $\triangleright$ Abb. 2.53)

Tab. 2.1 Produkteigenschaften.

\section{Eigenschaften der aufgezählten Produkte:}

\begin{tabular}{|c|c|c|}
\hline$\Delta-\Delta \Delta$ & 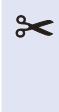 & $\begin{array}{l}\text { n:: } \\
\text { nur bei nicht } \\
\text { haftendem Biatain }\end{array}$ \\
\hline
\end{tabular}

- Biatain Silicone 7,5 ×7,5cm, $10 \times 10 \mathrm{~cm}, 10 \times 20 \mathrm{~cm}, 12,5 \times 12,5 \mathrm{~cm}$, $15 \times 15 \mathrm{~cm}, 17,5 \times 17,5 \mathrm{~cm}$ ( $\triangleright$ Abb. 2.54)

Tab. 2.2 Produkteigenschaften.

\section{Eigenschaften des oben genannten Produkts:}

000 sk-5, max.7T.

\section{Gelistet in den Helios-Kliniken}

mit schwacher Exsudataufnahme

- Biatain Silicone Lite $5 \times 5 \mathrm{~cm}, 5 \times 12,5 \mathrm{~cm}, 7,5 \times 7,5 \mathrm{~cm}, 10 \times 10 \mathrm{~cm}$, $12,5 \times 12,5 \mathrm{~cm}(\triangleright$ Abb. 2.55$)$

Tab. 2.3 Produkteigenschaften.

Eigenschaften des oben genannten Produkts:

○) $83-5, \max .7 \mathrm{~T}$.

\section{Vergleichbare Produkte}

- Mepilex, Mepilex border, Mepilex Border Lite, Mepilex Sacrum (MÖL)

- Allevyn, Allevyn Lite, Allevyn Gentle Border, Allevyn Gentle Border Lite, Allevyn Gentle Border Heel, Allevyn Gentle Border Sacrum, Allevyn Schaumverband haftend, Allevyn Sacrum, Allevyn Plus Sacrum (S\&N)

- Tielle, Tielle Lite, Tielle Hydropolymer Adhesive Dressing, Tielle Plus Hydropolymer Adhesive Dressing, Tielle Non-Adhesive Hydropolymer Dressing, Tielle Silicone Border Hydropolymer Adhesive Dressing, Tielle Packing Hydropolymer Dressing (zuschneidbar), Tielle Sacrum Hydropolymer Adhesive Dressing, Tielle Plus Sacrum Hydropolymer Adhesive Dressing, Tielle Plus Heel Hydropolymer Adhesive Dressing (SYS). 


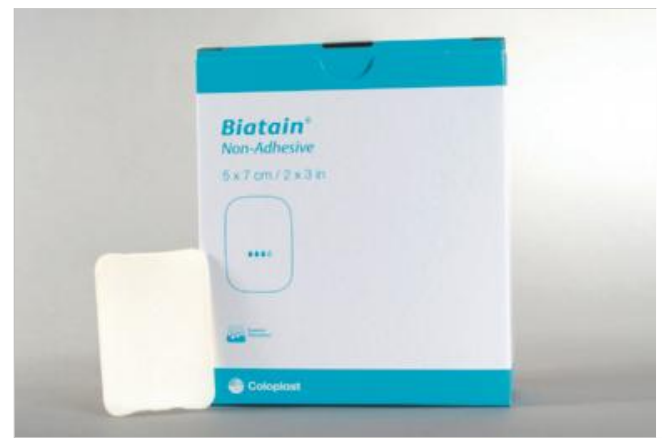

\section{Abb. 2.48 Biatain nicht} haftend.

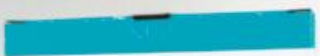

Abb. 2.49 Biatain selbsthaftend.

Biatain

Mdhesive

$125 \times 12.5 \mathrm{~cm} / 5 \times 5 \mathrm{~s}$

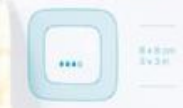

Scologtont

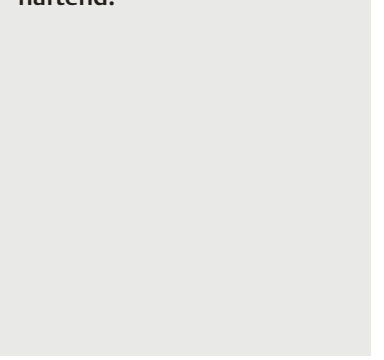

Abb. 2.50 Biatain-lbu.

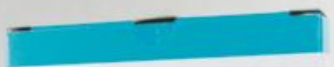

Biatain'Ibu

Non-Adhesive

$10 \times 10$ an $/ 4 \times 4$ in 


\section{Wundprodukte}

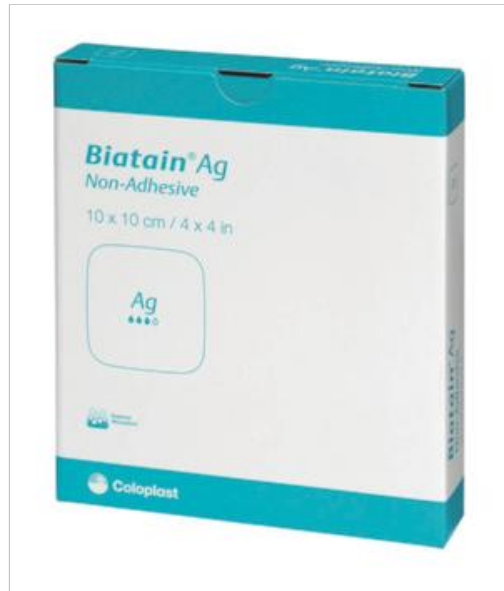

\section{Abb. 2.51 Biatain nicht haftend mit}

Silber. (Quelle: Mit freundlicher Genehmigung der Coloplast $\mathrm{GmbH}$ ) 


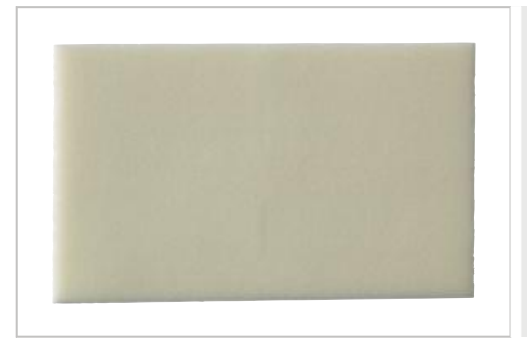

Abb. 2.53 Biatain Cavity. (Quelle: Mit freundlicher Genehmigung der Coloplast $\mathrm{GmbH}$ )

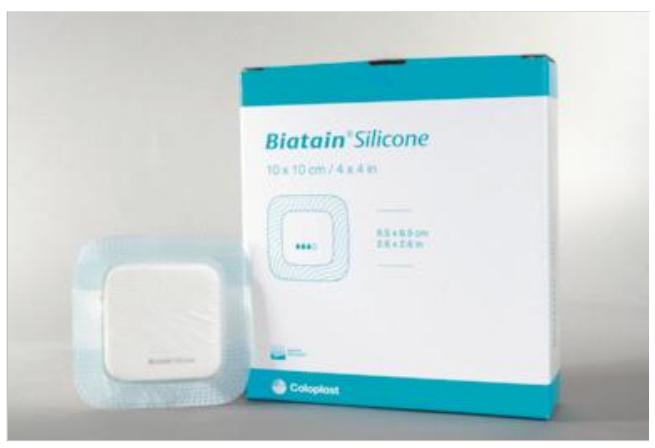

Abb. 2.54 Biatain Silicone.

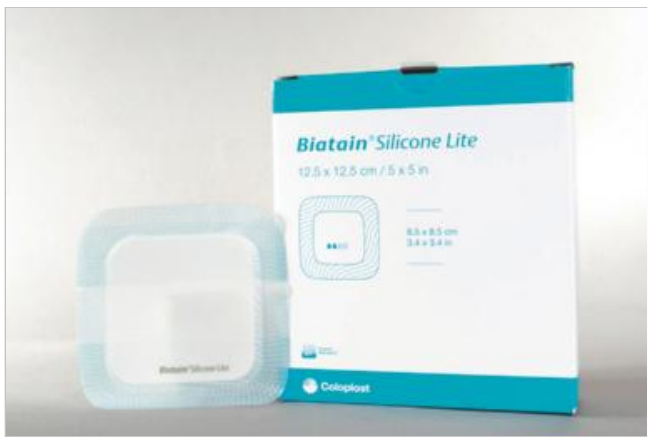

Abb. 2.55 Biatain Silicone Lite. 


\section{Beschreibung}

Hydropolymere bestehen aus chemisch und biologisch reizlosem Polyurethanschaum. Sie sind sehr flexibel und gut modellierbar. Sie besitzen eine hohe Exsudataufnahmefähigkeit, halten die Wundoberfläche feucht und ermöglichen einen freien Luft- und Wasserdampfaustausch. Sie verändern bei Exsudataufnahme nicht ihre Größe, wölben sich aber dem Wundgrund entgegen (1-2 $\mathrm{cm}$ bei Biatain). Bei Wunden bis $2 \mathrm{~cm}$ Tiefe ist daher nicht immer ein zusätzlicher Wundfüller erforderlich.

Siliconprodukte haben einen hautfreundlicheren Kleber und sind mehrfach positionierbar.

Als Besonderheit ist in Biatain-Ibu Ibuprofen als Analgetikum eingearbeitet.

Aufbau s. - Abb. 2.56, > Abb. 2.57, > Abb. 2.58, > Abb. 2.59.

\section{Indikation}

- Wunden mit schwach bis starker Exsudation.

- Bei schwach bis mittelstark exsudierenden Wunden eignen sich Verbände mit geringerer Schaumkissenstärke (Biatain Lite).

- Nicht haftende Schaumverbände sind geeignet für Wunden mit besonders fragiler Umgebungshaut.

- Selbsthaftende Schaumverbände werden eingesetzt bei Wunden mit intakter Umgebungshaut.

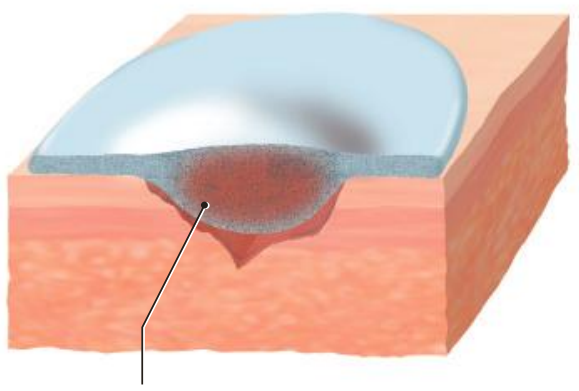

Abb. 2.56 Biatain: Funktion.

Schaum, der sich der Wunde anpasst. 


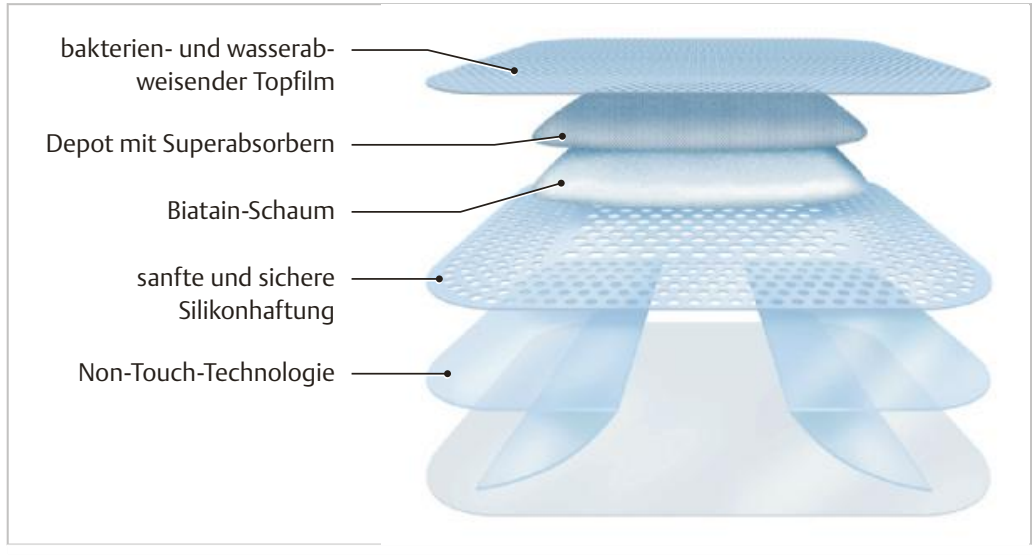

Abb. 2.57 Biatain Silicone: Aufbau.

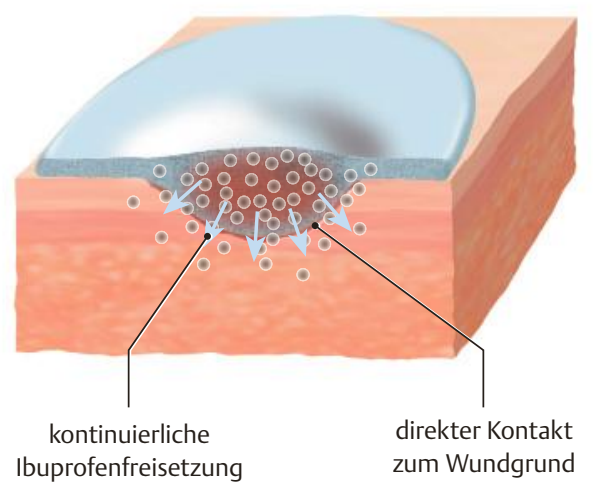

Abb. 2.58 Biatain-Ibu: Funktion.

- Silikonbeschichtete Auflagen haften sanft bei verletzlicher Umgebungshaut und sind repositionierbar.

- Absorbierende Schaumverbände eignen sich zum Einlegen in exsudierende tiefe oder höhlenförmige Kavitäten (Biatain Cavity). 


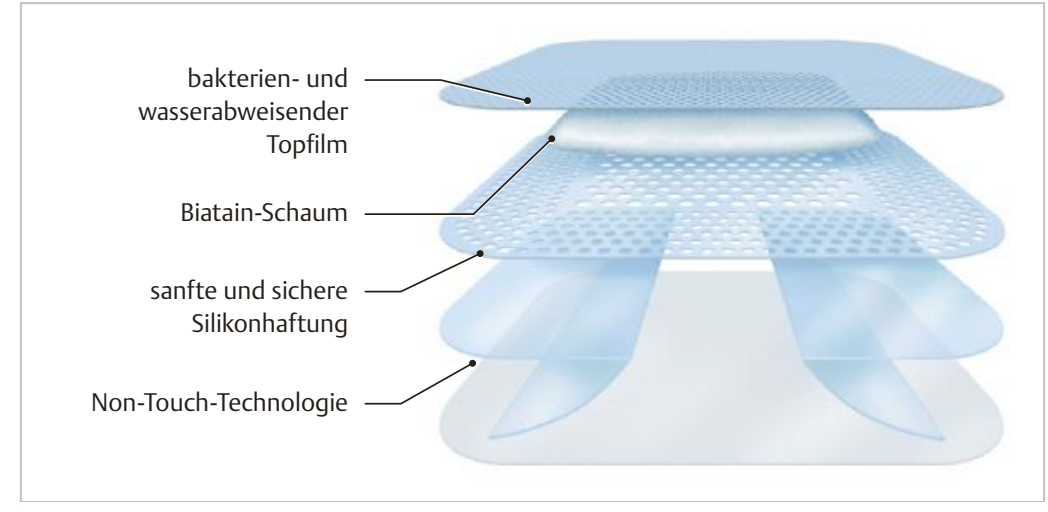

Abb. 2.59 Biatain Silicone Lite: Aufbau.

\section{Kontraindikationen}

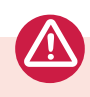

- Nicht bei trockenen Wunden anwenden!

- Massiv infizierte Wunden und zähflüssiges oder eitriges Exsudat können die Poren des Schaumes verstopfen $\rightarrow$ Okklusion.

\section{Anwendungshinweise}

- Nicht haftende Schäume werden 1,5-3 cm den Wundrand überragend zugeschnitten $\rightarrow$ sterile Schere verwenden!

- Cavity-Schäume müssen z. B. durch eine semipermeable Folie abgedeckt werden. Zur besseren Anpassung an die Wundoberfläche bei Exsudataufnahme ist es möglich, diese zu einer Spirale zu schneiden. Je nach Exsudatmenge die Wunde nur zur Hälfte bis zu drei Viertel mit dem Produkt ausfüllen, da sonst durch die Volumenzunahme Druck auf die Wundoberfläche ausgeübt wird.

- Bei Verwendung von Biatain cavity muss die Anzahl der eingelegten Schäume dokumentiert werden.

- Biatain Silicone: sehr flexibel und gut modellierbar, minimiert das Risiko von Mazeration, repositionierbar, unter Kompression geeignet, sanfte und sichere Silikonhaftung, keine Kleber (Produkte ohne Kleberand). 


\section{Merke}

\subsubsection{Hydropolymerverbände mit Superabsorber}

\section{Empfohlene Verwendung}

Für mittlere bis stark exsudierende Wunden. Ein Haftrand auf Hydrokolloidbasis ermöglicht ein sicheres Fixieren (Biatain Super). Gute Flüssigkeitsaufnahme durch ein hochsaugfähiges hydrokapillares Wundkissen mit einem semipermeablen, wasser- und bakteriendichten Topfilm. Auf der Wundseite verhindert eine Wundkontaktschicht ein Verkleben mit dem Wundgrund und erleichtert einen atraumatischen Verbandwechsel.

Aufbau s. $>$ Abb. 2.60 und $>$ Abb. 2.61.

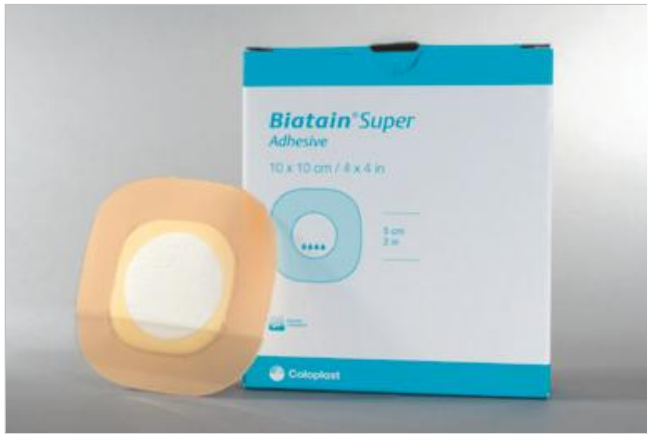

Abb.2.60 Biatain Super. 


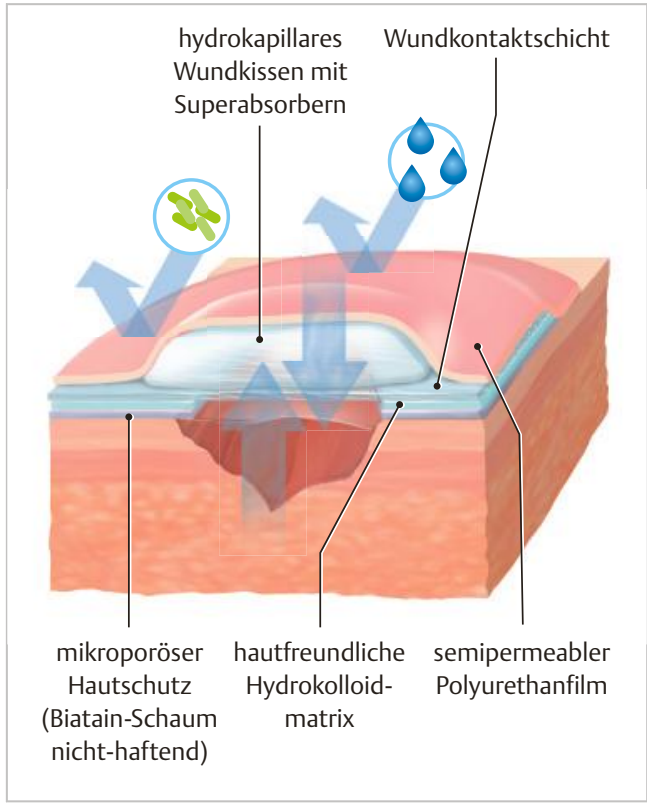

Abb. 2.61 Biatain Super:

Funktion.

\section{Produkte}

\section{Gelistet in den Helios-Kliniken}

Biatain Super selbsthaftend (COL) $10 \times 10 \mathrm{~cm}, 15 \times 15 \mathrm{~cm}$.

Tab. 2.4 Produkteigenschaften.

\section{Eigenschaften des oben genannten Produkts:}

000 s $34-6, \max .7 \mathrm{~T}$.

\section{Vergleichbare Produkte}

Siehe Kap. 2.8. 


\section{Beschreibung}

- Superabsorber-Partikel absorbieren und speichern große Mengen Wundexsudat. Fasern verteilen das Wundexsudat im gesamten Wundkissen und maximieren so die Absorption. Die hohe Durchlässigkeit für Wasserdampf trägt zum exzellenten Wundexsudatmanagement bei.

- Kompressionsstabil.

- PU-Schaum mit der zurzeit höchsten Flüssigkeitsaufnahmekapazität.

\section{Indikation}

Wunden mit mittlerer bis starker Exsudation.

\section{Kontraindikationen}

- Wunden mit freiliegenden Sehnen oder Knochen (Austrocknung!)

- Verbrennungen Grad 3 (Austrocknung!).

\section{Anwendungshinweise}

Siehe Kap. 2.7.2.

Bei Produkten mit Kleberand ist in Ausnahmefällen ein dachziegelartiges Aufbringen möglich, wenn ein Kleberand abgeschnitten und ein zweiter Hydropolymerverband überlappend angebracht wird.

Verbandwechsel: Normalerweise genügt ein Verbandwechsel alle 2-3 Tage (maximal 7 Tage). Bei häufigeren Wechseln ist alternativ eine Saugkompresse bzw. ein Kissen mit Superabsorber zu wählen.

\section{Merke}

Bei täglichem Verbandwechsel ist die Anwendung von Schaumstoffkompressen mit Superabsorber zu teuer. 


\subsubsection{Offenporige Hydropolymerverbände}

\section{Beschreibung}

Offenporige Kompressen führen zum Einsprossen von Kapillaren in den Schaum, daher eignen sie sich zur Wundkonditionierung vor Transplantation (Epigard s. Kap. 2.9.7 Hautersatzprodukte) und zur vorübergehenden Wundbehandlung (Ligasano).

\section{Epigard zur Wundkonditionierung vor Transplantation}

\section{Produkte}

\section{Gelistet in den Helios-Kliniken}

Epigard (BIV) $8 \times 5 \mathrm{~cm}, 8 \times 10 \mathrm{~cm}, 8 \times 23 \mathrm{~cm}, 12 \times 30 \mathrm{~cm}, 12 \times 45 \mathrm{~cm}$

( $\triangleright$ Abb. 2.62).

Siehe Kap. 2.9.7 Hautersatzprodukte.

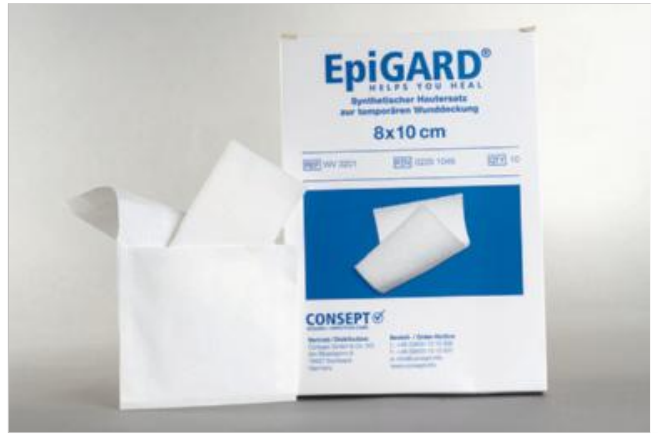

Abb. 2.62 Biovision Epigard. 


\section{Wundbehandlung mit PUR-Schaum (mittel- bis grobporig, gemischt- und offenporig)}

\section{Produkte}

\section{Gelistet in den Helios-Kliniken}

- Ligasano weiß steril (LIG): $10 \times 10 \times 1 \mathrm{~cm}, 15 \times 10 \times 1 \mathrm{~cm}, 6 \times 2,5 \times 0,4 \mathrm{~cm}$, $100 \times 1,5 \times 0,4 \mathrm{~cm}, 300 \times 2,5 \times 0,4 \mathrm{~cm}, 5 \times 5 \times 1 \mathrm{~cm}, 29,5 \times 24,5 \times 1 \mathrm{~cm}$ ( $\vee$ Abb. 2.63)

- Ligasano grün steril (LIG) $15 \times 10 \times 2 \mathrm{~cm}$ ( $\triangleright$ Abb. 2.64).

Sonderformen steril:

- „T“-Verband $14 \times 10 \times 0,5 \mathrm{~cm}$

- Fersenverband steril $\emptyset 20 \times 1 \mathrm{~cm}$

- Hallux-valgus-Verband steril 4,0/4,5× $12 \mathrm{~cm}$;

- Zehenschläuche 3,5/2,5×6 cm (groß), 2,5/1,5×6 cm (klein), 1,5×6 cm, ( $\vee$ Abb. 2.65)

- Ligasano Wundputzer medium steril $5 \times 5 \times 2 \mathrm{~cm}$ orange ( $\triangleright$ Abb. 2.66).

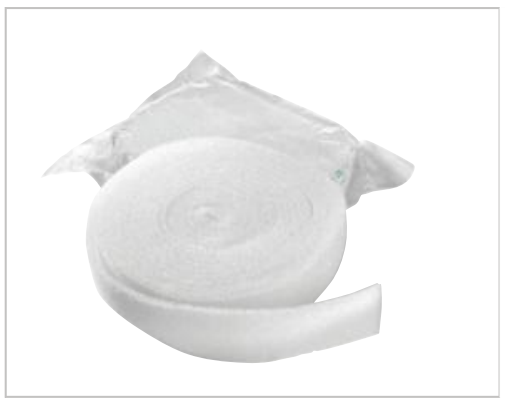

Abb. 2.63 Ligamed Ligasano weiß steril. (Quelle: Mit freundlicher Genehmigung der Ligamed medical Produkte $\mathrm{GmbH}$ )

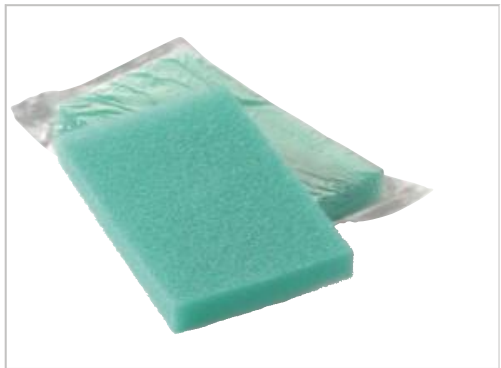

Abb. 2.64 Ligamed Ligasano grün steril. (Quelle: Mit freundlicher Genehmigung der Ligamed medical Produkte $\mathrm{GmbH}$ ) 

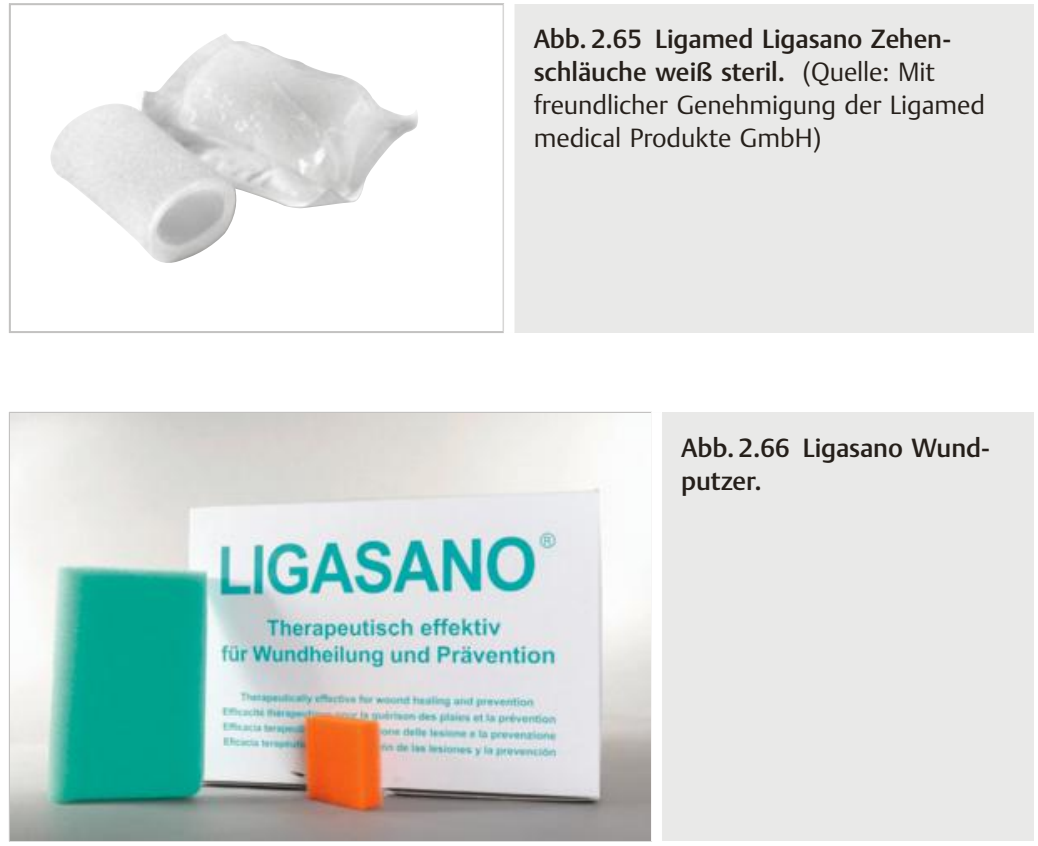

Abb. 2.66 Ligasano Wundputzer.

\section{Beschreibung}

Ligasano weiß ist ein PUR-Schaumstoff mit breitem Anwendungsspektrum zur Wundreinigung, Tamponade und Wundabdeckung (steril) sowie Polsterung $\rightarrow$ Druckverteilung (unsteril). Es eignet sich durch einen intensiven Reinigungseffekt für verschmutzte, exsudierende und infizierte Wunden ( $\triangleright$ Abb. 2.67).

\section{Wirkungsweise}

- Mechanischer Reiz: wirkt lokal durchblutungsfördernd $\rightarrow$ dadurch bessere Nähr- und Sauerstoffversorgung im Wundgebiet. Der mechanische Reiz hält bis zu 3 Tagen an, dann Verbandwechsel.

- Geringe Druckspannung: kompressionsarme Anpassung an Konturen, gleichmäßige, reduzierte Druckbelastung auf Wunde und Körper.

- Gezielte Saugwirkung: Flüssigkeitsüberschuss wird aufgesaugt, ohne austrocknend zu wirken. Feuchtwarmes Wundmilieu, Keimreduzierung, Wund- 
reinigung, kein Verkleben mit der Wunde. Vermeidung von Hautmazeration, Hautpflege.

Übersicht s. ^ Abb. 2.68 .

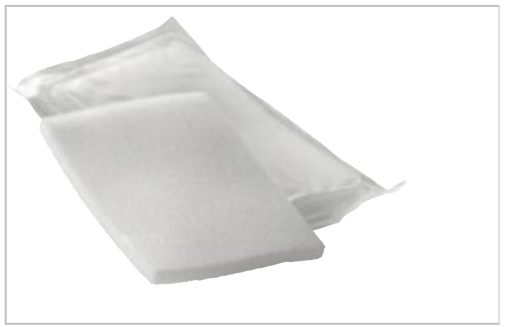

Abb. 2.67 Ligasano weiß. (Quelle: Mit freundlicher Genehmigung der Ligamed medical Produkte $\mathrm{GmbH}$ )

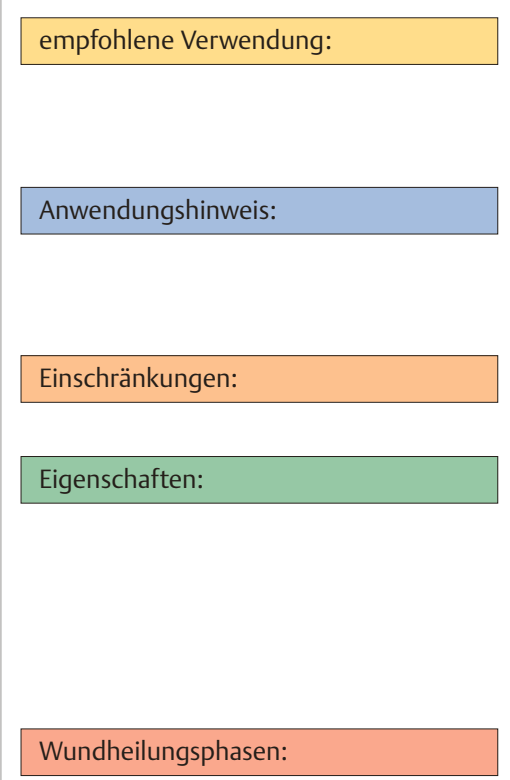

- Wundreinigung (Lösen von Fibrinbelägen/Biofilm)

- Wundkonditionierung

- Prävention

- zuschneid- und tamponierbar

- formstabil, quillt nicht

- Sekundärverband inkl. Polsterung

- Anwendung nur mit Schulung

- nicht auf Knochen oder Sehnen legen

- kein direkter Organkontakt

- nachlassende Druckspannung

- mechanischer Reiz/Wundreinigung durch Selbstreinigung

- kontrollierte Saugwirkung

- Wasserdampfpermeabilität

- allergieneutral

○ 1:: $\bigcirc 1-2, \max .3 T$.

- vorwiegend Reinigungsphase

- Granulationsphase

Abb. 2.68 Hydropolymerverbände offenporig. 


\section{Eigenschaften}

- Schnell nachlassende Druckspannung: reduziert den Druck auf die Wunde, fördert die Granulation und reduziert den Gegendruck auf das neue Granulationsgewebe.

- Mechanischer Reiz: aktiviert die Selbstreinigung und fördert Durchblutung und Granulation.

- Kontrollierte Saugwirkung: nur überschüssiges Exsudat wird aufgenommen und vertikal weitergeleitet. Auch Debris/Zelltrümmer können über die grobe Struktur aufgenommen werden.

\section{Indikation}

- in allen Wundphasen und Wundarten einsetzbar

- mechanisches Débridement

- Granulationsförderung

- sekundär heilende Wunden

- präoperative Wundkonditionierung (Exsudatabfluss muss gewährleistet sein!)

- Fistelgänge, Wundhöhlen/-taschen, Unterminierung

- infizierte Wunden - in Kombination mit Antiseptika wie Polyhexanid etc.

- Spalthauttransplantation

- Verbrennungen (Grad 1-3) in offener oder geschlossener Versorgung.

\section{Cave}

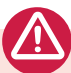

- Einsprossen von z. B. Granulationsgewebe ins Material ist möglich, wenn Verbandwechsel nicht zeitgerecht erfolgt.

- Die Auflage darf ohne Schutz durch Hydrogele nicht direkt auf Knorpel, Knochen, Periost, Perichondrium oder Sehnen angewendet werden.

\section{Anwendungsweise}

- Ligasano weiß kann zugeschnitten (sterile Schere!) und in die Wunde eingelegt werden:

- etwas größer als Wunddurchmesser zuschneiden

- mit leichter Kompression (Material quillt nicht) einbringen, um überall zuverlässig einen Kontakt herzustellen.

- Bei Gewebszerstörung im Subkutisbereich besteht bei Anwendung Austrocknungsgefahr und somit die Gefahr der Nekrosenbildung! 
- Es können Schmerzen beim Verbandwechsel sowie Verletzungen am Granulationsgewebe aufgrund des Adhäsionseffekts des offenporigen Schaumverbandes auftreten.

- Luftoffene Fixierung mit Fixierhöschen, Schlauchverband, Mullbinde etc.

\section{Sekundärabdeckung:}

- bei geringer bis mäßiger Exsudation: Ligasano weiß

- bei starker Exsudation: Superabsorber, Saugkompresse.

\section{Verbandwechsel:}

- einmal täglich bis alle 3 Tage; spätestens, wenn an der Außenseite der erste Exsudatpunkt sichtbar ist

- alle 1-2 Tage bei Fistelversorgungen

- aufgrund der zelligen Struktur ist ein Einsprossen von z. B. Granulationsgewebe ins Material möglich (vorbeugend frühzeitiger Verbandwechsel)

- mehrere Tage bei Wundkonditionierung.

\section{Cave}

Verklebung! Nur weißer Schaum darf auf die Wunde/Haut.

Grüner und oranger Schaum: Wund- und Hautkontakt ist zu vermeiden; möglich in Kombination mit weißem Schaum.

\subsection{Superabsorber}

\section{Produkte}

\section{Gelistet in den Helios-Kliniken}

- RespoSorb Super $10 \times 10 \mathrm{~cm}, 10 \times 20 \mathrm{~cm}, 20 \times 25 \mathrm{~cm}, 20 \times 40 \mathrm{~cm}$ (HAR) ( $\triangleright$ Abb. 2.8)

- Biatain Super selbsthaftend $10 \times 10 \mathrm{~cm}, 15 \times 15 \mathrm{~cm}(\mathrm{COL})(\triangleright$ Abb. 2.60)

- Eclypse Contour $30 \times 51 \mathrm{~cm}$, Eclypse Boot ( $\vee$ Abb. 2.69) $55 \times 47 \mathrm{~cm}$, $60 \times 70 \mathrm{~cm}, 70 \times 80 \mathrm{~cm}$, Eclypse Foot ( $\vee$ Abb. 2.70) $33 \times 48 \mathrm{~cm}$ (ADV).

Tab. 2.5 Produkteigenschaften.

\section{Eigenschaften der oben genannten Produkte:}

100 अ




\section{Vergleichbare Produkte}

- Askina Absorb + (BBR)

- Cutisorb Ultra, Cutimed Sorbion Sachet Border, Cutimed Sorbion Sachet Extra, Cutimed Sorbion Sachet Multi Star, Cutimed Sorbion Sachet S, Cutimed Sorbion sana, Cutimed Sorbion Sachet XL (BSN)

- Duramax, Exu-Dry, Allevyn Life (S\&N)

- HydroClean (HAR)

- Mextra Superabsorbent (MÖL)

- ROGG Sorb lite (ROG) ist zuschneidbar

- Tegaderm Superabsorber (3M)

- UrgoSuperabsorber, UrgoSuperSuperabsorber (URG)

- Vliwasorb Pro (L\&R)

- Zetuvit plus (HAR).

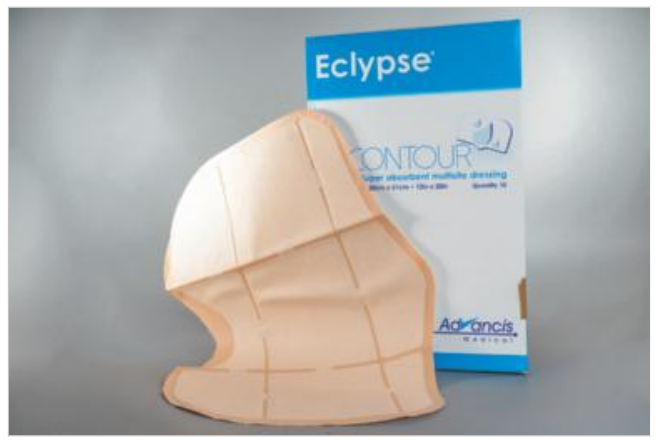

Abb. 2.69 Eclypse Boot.

\section{Eclypse Foot}

Abb. 2.70 Eclypse Foot.
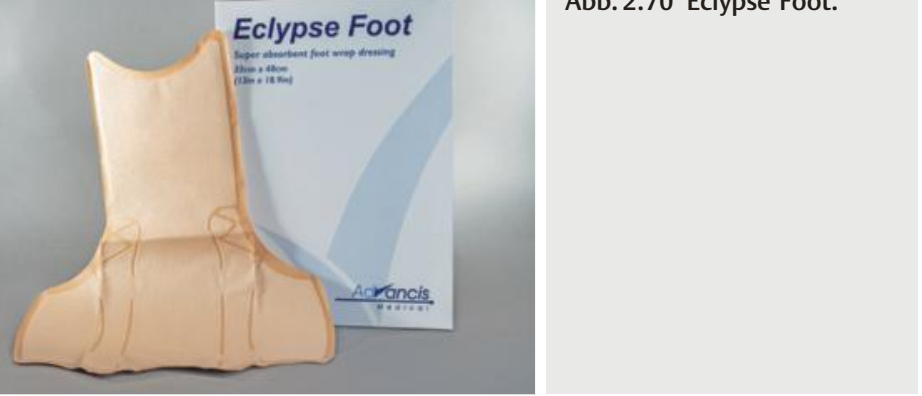


\subsubsection{Beschreibung}

Superabsorber sind Kunststoffe, die ein Vielfaches ihres Eigengewichts an Flüssigkeiten aufsaugen können. Man findet sie größtenteils in der Inkontinenzversorgung, in Babywindeln, Damenhygieneprodukten und in Verbandmaterial.

Der Superabsorber quillt bei Flüssigkeitsaufnahme auf und bildet ein Hydrogel.

Siehe auch die Beschreibung von RespoSorb Super in Kap. 2.2.2 und Biatain Super in Kap. 2.7.2.

Eigenschaften:

- hohe Flüssigkeitsaufnahme unter Aufrechterhaltung eines feuchten Wundmilieus

- vertikale Exsudataufnahme

- Aufnahme von einigen Hundert Milliliter Flüssigkeit, dabei formstabil, jedoch starkes Aufquellen $\rightarrow$ Achtung dadurch Druckbelastung der Wunde möglich

- längere Wundruhe durch größere Wechselintervalle.

\subsubsection{Anwendungshinweise}

- Geeignet für stark exsudierende Wunden.

- Teilweise, je nach Hersteller auch unter Kompression anwendbar.

- Auflage muss den Wundgrund berühren.

- Kombinationen mit anderen Wundauflagen wie z. B. Wunddistanzgitter, Alginat etc. sind möglich.

- Eclypse Wundauflagen mit Superabsorber sind für großflächige nässende Wunden an Bein, Fuß und Rumpf erhältlich. Die Auflage besteht aus einem Polyester- und Viskoseteil für eine schnelle Flüssigkeitsaufnahme. Im Inneren befindet sich ein Kissen aus hochsaugfähigen Kristallen in mechanisch gebundener Zellulose. Die Außenschicht hat eine hohe MVTR („Moisture Vapor Transmission Rate“ = Wasserdampfdurchlässigkeit), ist wasserabweisend und schützt vor einem Austreten des Exsudats.

- Das betroffene Körperteil wird mit der weißen Seite nach innen in den Verband gepackt. Die Befestigung erfolgt mit Klebestreifen oder Binden.

Anwendung von Eclypse Foot s. > Abb. 2.71, Eclypse Contour s. \ Abb. 2.72.

\section{Kontraindikationen}

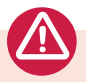

- keine Anwendung an Schleimhäuten oder Augen

- keine Anwendung bei trockenen Wunden, da weitere Austrocknung

- keine Anwendung auf Knochen, Knorpel oder Sehnen. 


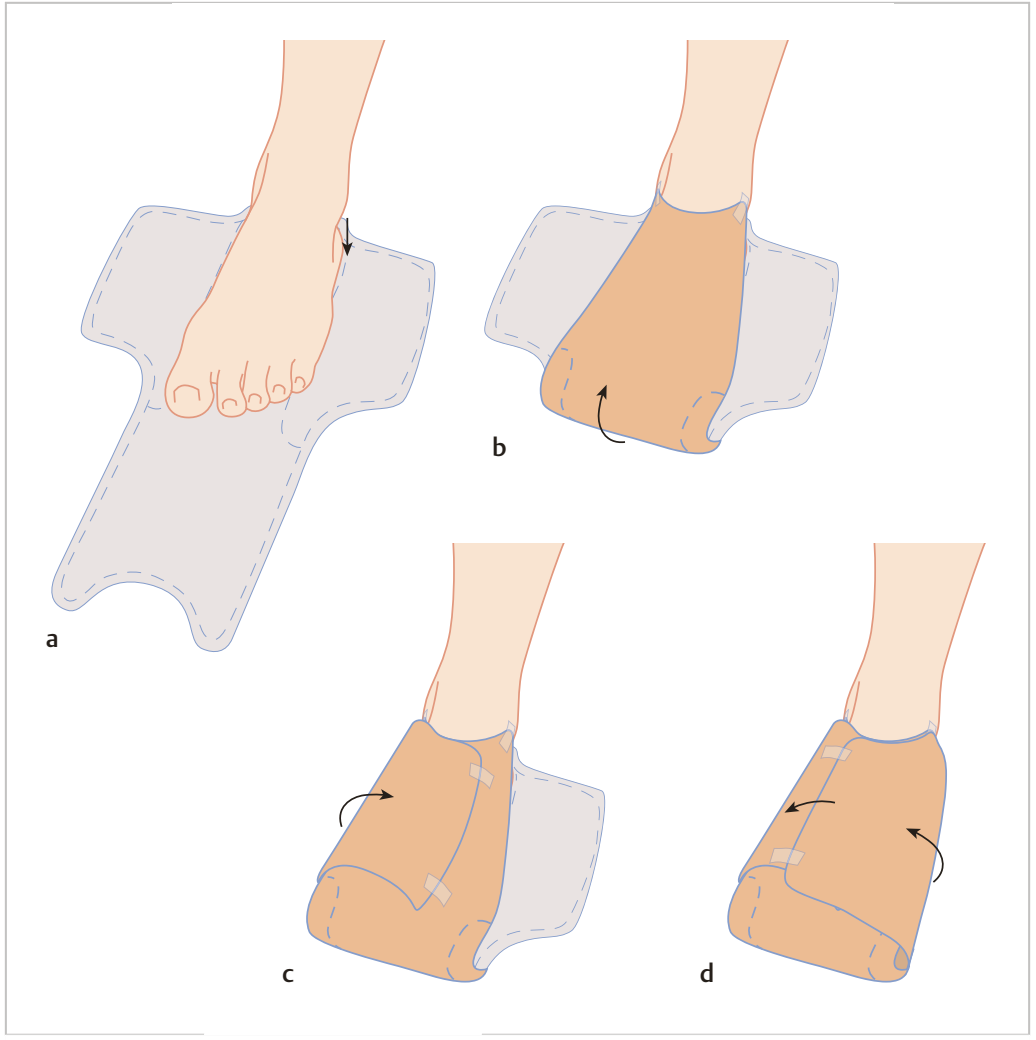

Abb. 2.71 Eclypse Foot: Anwendung. a-d

\section{Cave}

Nicht zerschneiden oder öffnen, da sich herausfallende Polymere in der Wunde festsetzen. 


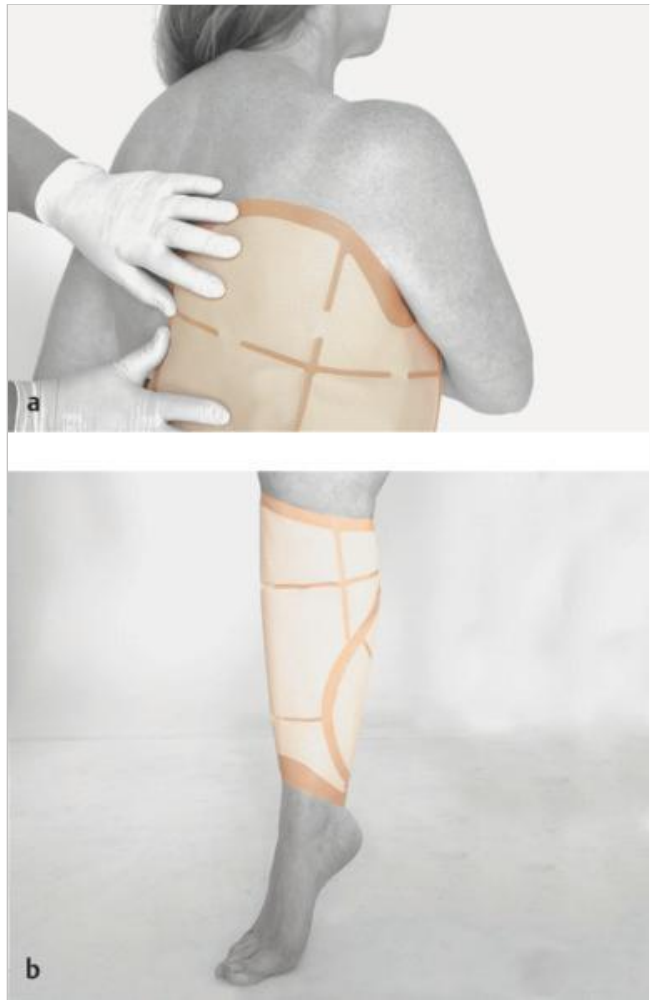

Abb. 2.72 Eclypse Contour:

Anwendung. (Quelle: Mit

freundlicher Genehmigung der Advancis medical $\mathrm{GmbH}$

Deutschland)

a Anwendung am Rumpf.

b Anwendung am Unterschenkel.

\section{Sekundärabdeckung:}

Fixation mit Binden, TG-Schlauch oder Folie.

Bei Fixierung mit Folie oder Fixomull die Ausdehnung berücksichtigen, sonst kommt es zu Spannungsblasen $\rightarrow$ Folie muss dehnbar sein.

Verbandwechsel: Verweildauer je nach Exsudatmenge 2-7 Tage. 


\subsection{Spezielle Produkte}

\subsubsection{Hydrophobe Wundauflagen}

\section{Produkte}

\section{Gelistet in den Helios-Kliniken}

- Cutimed Sorbact (BSN) ( $\vee$ Abb. 2.73)

- Cutimed Sorbact Kompressen $7 \times 9 \mathrm{~cm}$

- Cutimed Sorbact Tamponaden $2 \times 50 \mathrm{~cm}, 5 \times 200 \mathrm{~cm}$.

\section{Vergleichbare Produkte}

Keine.

\section{Nicht gelistet}

Kombinationsprodukte:

- Cutimed Sorbact NPWT Wundfüller

- Cutimed Sorbact Gel

- Cutimed Sorbact Hydroaktive B

- Cutimed Siltec Sorbact

- Cutimed Sorbion Sorbact

- Cutimed Saugkompressen

- Cutimed Sorbact Tupfer.
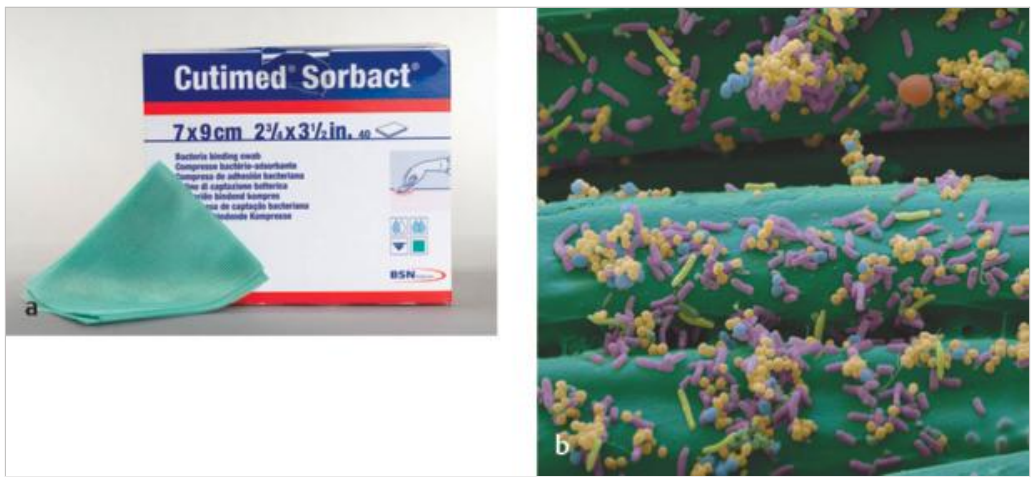

Abb. 2.73 Cutimed Sorbact.

a Verpackung und ausgepacktes Produkt.

b Mikroskopische Ansicht der Produktoberfläche. (Quelle: Mit freundlicher Genehmigung der BSN medical GmbH) 


\section{Beschreibung}

- Wundverband mit rein physikalischer Wirkweise zur Infektbekämpfung.

- Er bindet Keime durch hydrophobe (wasserabweisende) Wechselwirkung mit der Bakterienwand. Diese Bindung an die Wundauflage ist irreversibel.

- Kein Einsatz chemischer antimikrobieller Substanzen.

- Mit jedem Verbandwechsel wird die Keimzahl in der Wunde reduziert.

- Es ist keine bekannte Resistenzentwicklung bei Bakterien oder Pilzen beschrieben.

- Keine Freisetzung von Endotoxinen, da kein Abtöten von Bakterien und Pilzen erfolgt.

Übersicht s. - Abb. 2.74 .

\section{Indikation}

- kritisch kolonisierte und infizierte Wunden

- Fisteln und Abszesse nach chirurgischem Débridement/Spaltung

- postoperative Wunden und Wunddehiszenzen

- Hautpilzinfektionen.

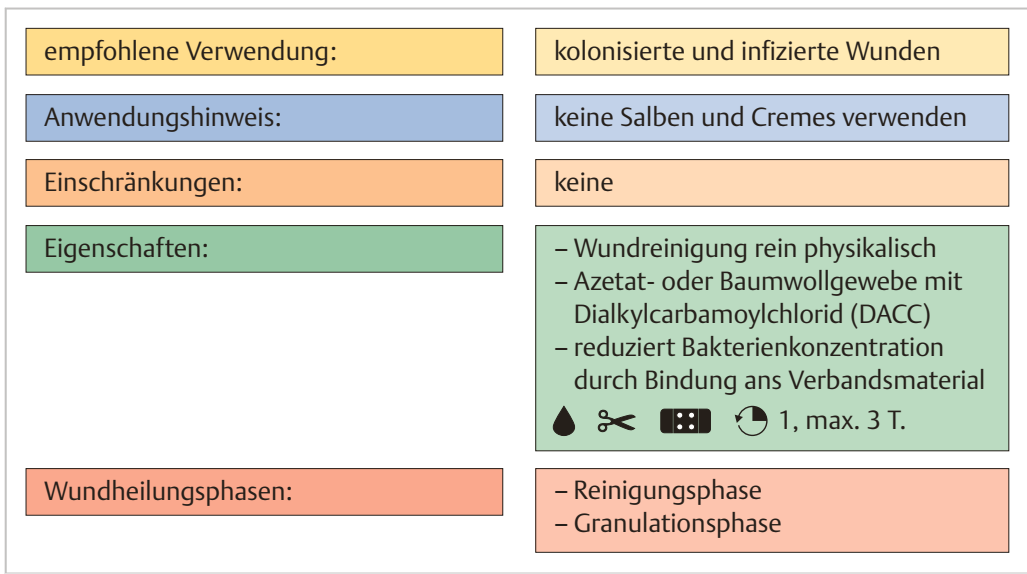

Abb. 2.74 Hydrophobe Wundauflagen. 


\section{Kontraindikationen}

- keine bekannten Gegenanzeigen

- keine bekannten Allergierisiken.

\section{Anwendungsweise}

- Wundreinigung kontaminierter und infizierter Wunden durch DACC mittels hydrophober Wirkung auf rein physikalischer Basis.

- Keine Salben und Cremes zusätzlich verwenden (Aufhebung der hydrophoben Wirkung).

Sekundärabdeckung: Sekundärverband abhängig von der Exsudation notwendig.

Verbandwechsel: Alle 1-3 Tage.

\subsubsection{HydroBalance-Wundverband}

\section{Produkte}

\section{Gelistet in den Helios-Kliniken}

Suprasorb X+PHMB 5×5, $9 \times 9 \mathrm{~cm}($ L\&R) $(\triangleright$ Abb. 2.75).

\section{Nicht gelistet}

Suprasorb X.

\section{Vergleichbare Produkte}

Keine. 


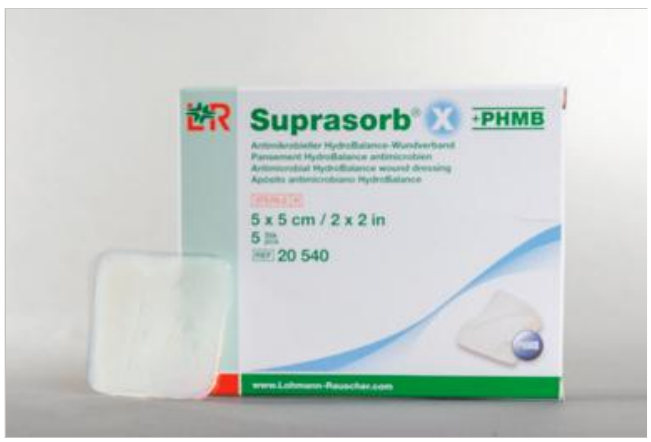

Abb. 2.75 Suprasorb $X$ PHMB.

\section{Beschreibung}

Suprasorb X ist ein feuchtigkeitsregulierender Wundverband für nicht infizierte Wunden.

Gelistet in den Helios-Kliniken ist Suprasorb $\mathrm{X}+\mathrm{PHMB}$. Dies ist ein antimikrobieller Wundverband, der gegen ein breites Erregerspektrum, wie z.B. MRSA und VRE wirkt. Innerhalb von 24 Stunden wird in vitro eine sehr schnelle und fast vollständige Freisetzung des Polyhexanids (PHMB) aus der Wundauflage gezeigt.

Durch die Struktur der biosynthetischen HydroBalance-Fasern wird je nach Wundzustand Feuchtigkeit an die Wunde abgegeben oder überschüssiges Exsudat aufgenommen. Dadurch entsteht für den Patienten eine Schmerzreduktion ohne pharmakologischen Zusatz und eine Steigerung der Lebensqualität.

Das elastische und strukturstabile Material erlaubt eine einfache Anpassung an jede Wundform und Wundtiefe und eignet sich deswegen besonders für schwer zu versorgende Körperstellen.

Übersicht s. > Abb. 2.76.

\section{Indikation}

Für die Versorgung infizierter Wunden, wenn diese schwach bis mittel exsudierend, oberflächlich oder tief sind, z. B.:

- arterielle und venöse Ulzera

- diabetische Ulzera

- Druckgeschwüre

- oberflächliche Verbrennungen 2. Grades 


\begin{tabular}{l}
\hline empfohlene Verwendung: \\
\hline Anwendungshinweis: \\
\hline
\end{tabular}

Einschränkungen:

Eigenschaften:

Wundheilungsphasen: kolonisierte und infizierte Wunden

bei Antrocknen Rehydrierung 10 min notwendig

PHMB-Unverträglichkeit

- Hydro-Balance-Fasern:

Überschüssiges Exsudat aus schwach exsudierender Wunde wird in Verband aufgenommen.

- Feuchtigkeit aus Verband wird in austrocknungsgefährdete Wunde abgegeben.

- Abtötung von Mikroorganismen durch PHMB

- Schmerzreduktion ohne pharmakologischen Zusatz und Steigerung der Lebensqualität

\& : $\bigcirc 3-5, \max .7 \mathrm{~T}$.

- Reinigungsphase

- Granulationsphase

Abb. 2.76 HydroBalance Wundverband.

- postoperative chirurgische Wunden

- Hauttransplantate

- Spalthautentnahmestellen

- Schürfwunden, Lazerationen (Hauteinrisse).

\section{Kontraindikationen}

Keine. 


\section{Anwendungsweise}

Die Wunde wird nach üblichem Standard gereinigt und getrocknet. Suprasorb $\mathrm{X}+\mathrm{PHMB}$ auf die Wunde auflegen und anmodellieren. Bei Verwendung der Tamponade diese mehrmals falten und locker in die Wunde einbringen.

\section{Cave}

Sollte der Verband antrocknen, muss eine ausreichende Rehydrierung mit einer Einwirkzeit von mindestens 10 Minuten vor der Entfernung erfolgen.

Sekundärabdeckung: Sekundärverband notwendig, abhängig von der Exsudation bei nicht infizierten Wunden mit Folie oder Schaumverband.

Verbandwechsel: Alle 3-5 Tage.

\subsubsection{Hydroaktive Wundauflagen - Laminate - HydroClean}

Wundkissen mit Saugspülmechanismus ohne antiseptische Wirkung

\section{Produkte}

\section{Nicht in den Helios-Kliniken gelistet}

- HydroClean (HAR) (- Abb. 2.77)

- HydroClean Cavity (HAR) (für Wundhöhlen)

\section{Vergleichbare Produkte}

Keine.

Abb. 2.77 HydroClean. (Quelle: Mit freundlicher Genehmigung der Paul Hartmann AG) 


\section{Beschreibung}

- Umhüllung: Polypropylengestrick

- Quellkörper: Polyacrylat-Superabsorber in mit Ringerlösung aktiviertem Wundkissen

- auch unter Kompressionsverbänden anwendbar

- keine Verklebungen mit der Wunde

- Abgabe von Ringerlösung in die Wunde und Aufnahme von Bakterien, abgestorbenen Zellen und Toxinen

- feuchtigkeitsabweisende Schicht verhindert Durchnässen des Verbandes.

Übersicht s. $>$ Abb. 2.78 .

\section{Indikation}

- in der Reinigungsphase bei fibrinös belegten oder stark exsudierenden Wunden

- Nasstherapie von Wunden mit stagnierender Heilungstendenz.

\begin{tabular}{l}
\hline empfohlene Verwendung: \\
\hline Anwendungshinweis: \\
\hline Einschränkungen: \\
\hline
\end{tabular}

Eigenschaften:
- Wunden mit stagnierender Heilungstendenz

- infizierte Wunden

Hautschutz für Wundumgebung

keine Anwendung bei trockenen Nekrosen und trockener Gangrän (pAVKIV)

- Abgabe von Ringerlösung in die Wunde und Aufnahme von Bakterien, abgestorbenen Zellen und Toxinen - verklebt nicht mit Wunde

○) I:: $\bigcirc$ max.1-3T.

Abb. 2.78 Hydroaktive Wundauflagen. 


\section{Kontraindikationen}

- trockene Nekrosen

- trockene Gangrän (pAVK IV)

- späte Phasen der Wundheilung.

\section{Anwendungsweise}

- Hautschutz für Wundumgebung

- Produktgröße entsprechend der Wundgröße auswählen

- Kontakt mit dem Wundgrund erforderlich

- nicht schneidbar

- weiße Seite ohne grüne Striche wird auf die Wundfläche gelegt.

Sekundärabdeckung: Fixieren mit Fixierpflaster, Klebevlies oder Schlauchverband.

Verbandwechsel: Alle 1-3 Tage. Eventuell vor dem Verbandwechsel nochmals mit Ringerlösung tränken; erleichtert das Ablösen.

\section{Wundkissen mit Saugspülmechanismus mit antiseptischer Wirkung}

\section{Produkte}

Nicht in den Helios-Kliniken gelistet

HydroClean plus mit PHMB (HAR).

\section{Beschreibung}

Siehe oben, zusätzlich antibakteriell durch PHMB.

\section{Indikation}

Wunden mit stagnierender Heilungstendenz sowie stärker kolonisierte oder infizierte Wunden (breite antimikrobielle Wirksamkeit). 


\subsubsection{Kollagen}

\section{Kollagene ohne antiseptische Wirkung}

\section{Produkte}

\section{Gelistet in den Helios-Kliniken}

Keine Listung von Produkten ohne antiseptische Wirkung, stattdessen Produkte mit antiseptischer Wirkung, siehe unten.

\section{Vergleichbare Produkte}

- Promogran $28 \mathrm{~cm}^{2}, 123 \mathrm{~cm}^{3}$ (SYS)

- Cutimed Epiona (BSN)

- Nobakoll (NOB)

- ROGG Bio-Kollagen (ROG)

- Suprasorb C (L\&R).

\section{Beschreibung}

Kollagene Wundauflagen bestehen aus tierischen Kollagenen, Promogran enthält zusätzlich noch oxidierte, regenerierte Zellulose.

Kollagendispersionen werden gefriergetrocknet. Es entstehen poröse, schwammartige Wundauflagen mit mäßiger Saugkapazität für Exsudat und Zelltrümmer sowie blutstillenden Eigenschaften. Man nimmt an, dass das Kollagen durch die in der chronischen Wunde vorhandenen Proteasen abgebaut wird und die entstehenden Eiweißbruchstücke chemotaktisch Fibroblasten anziehen. Diese sollen zur eigenen Kollagensynthese anregen und den proteolytischen Abbau der körpereigenen Wachstumsfaktoren vermindern.

Das Kollagen wird vom Körper nach Tagen resorbiert.

Übersicht s. > Abb. 2.79.

\section{Indikation}

Stagnierende Wundheilung in der Granulationsphase.

\section{Kontraindikationen}

- Nicht bei Infektion einsetzen!

- Nicht auf Nekrosen auflegen!

- Nicht in Verbindung mit eiweißfällenden Substanzen wie Jod oder gerbenden Substanzen wie Silbernitrat anwenden! 


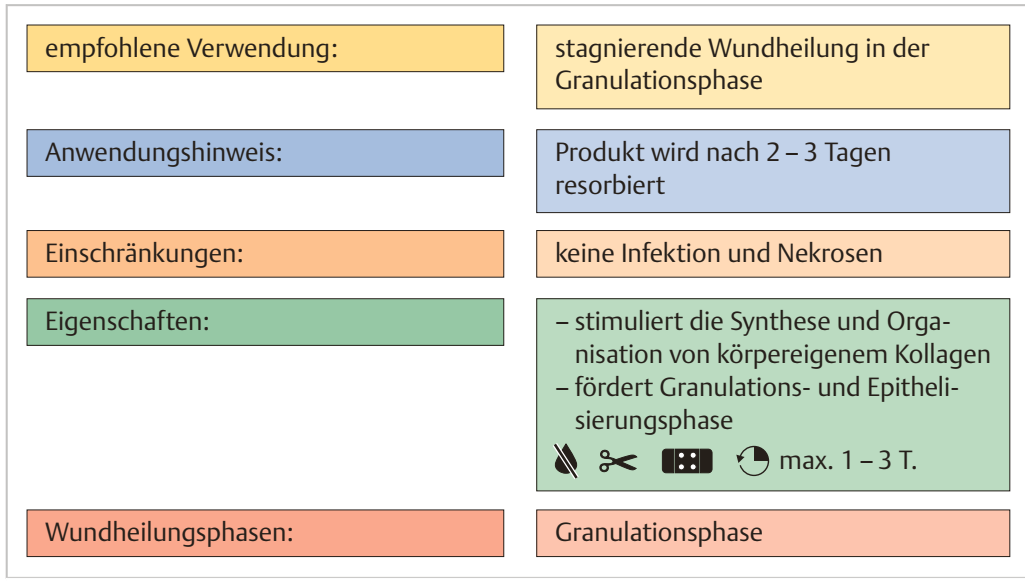

Abb. 2.79 Kollagene Wundauflagen.

\section{Anwendungsweise}

- Bei trockenen Wunden kann das Produkt mit physiologischer Kochsalzlösung angefeuchtet und die gelierte Wundauflage auf dem Wundgrund ausgelegt werden.

- Die Wundauflage wird mit einer sterilen Schere auf die Wundgröße zurechtgeschnitten oder gefaltet in tiefe Defekte mit Kontakt zum Wundgrund eingelegt.

\section{Sekundärabdeckung:}

- Notwendig; Sekundärverband je nach Exsudation auswählen: Saugkompressen, semipermeable Wundfolien, Hydrokolloide oder Schaumstoffkompressen.

- Bei tieferen Wunden nur den Wundgrund auslegen/modellieren, mit einem Wundfüller die Wunde ausfüllen und den Sekundärverband je nach Exsudation auswählen.

Verbandwechsel: Nach 2-3 Tagen, Materialreste werden beim Verbandwechsel nicht entfernt. 


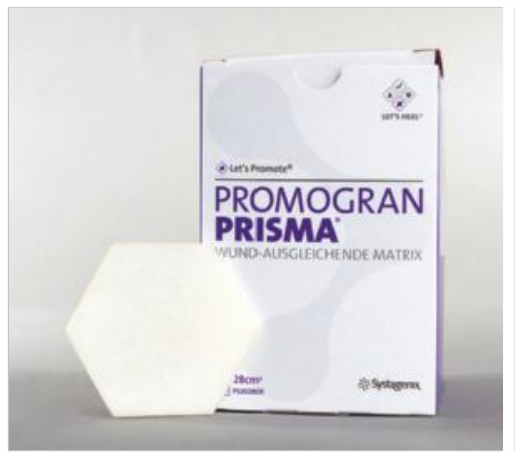

Abb. 2.80 Promogran Prisma.

\section{Kollagen mit antiseptischer Wirkung}

\section{Produkte}

\section{Gelistet in den Helios-Kliniken}

Promogran Prisma $28 \mathrm{~cm}^{2}, 123 \mathrm{~m}^{2}(\mathrm{KCl})(\triangleright$ Abb. 2.80).

\section{Beschreibung}

Promogran Prisma ist auf der Basis von Promogran hergestellt und enthält zusätzlich einen geringen Silberanteil. Damit wurde die Indikation auf kolonisierte und kritisch kolonisierte Wunden erweitert.

\subsubsection{Kohleprodukte}

\section{Einführung}

\section{Beschreibung}

Aktivkohlekompressen kombiniert mit mehrschichtigen stark saugenden Materialien wie Vlies, Viskose, Polyamid, Zellulose oder Polypropylen absorbieren starke Gerüche und binden Eiweißmoleküle. Bei der Anwendung ist zu prüfen, ob kausale therapeutische Maßnahmen wie chirurgisches Débridement und eventuell systemische Antibiotikagabe zusätzlich notwendig sind.

Übersicht s. - Abb. 2.81 . 


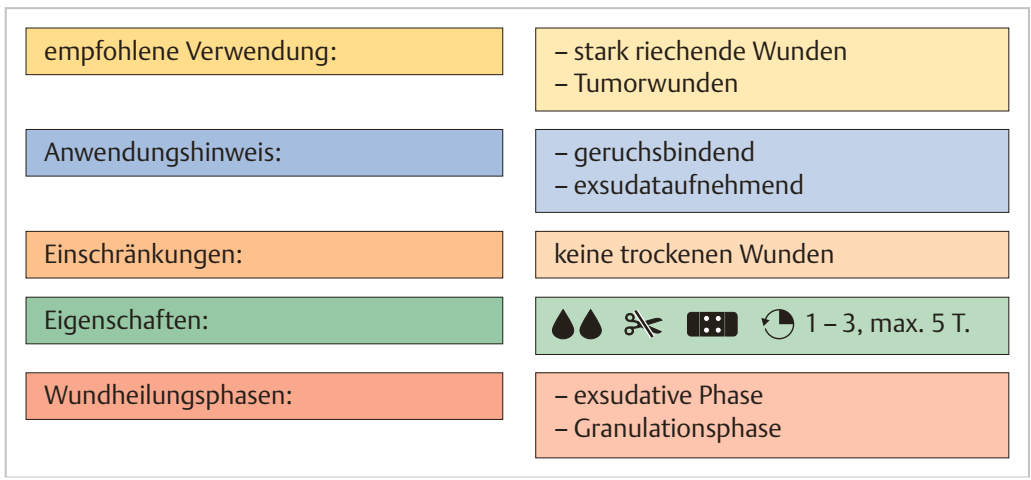

Abb. 2.81 Kohleprodukte.

\section{Indikation}

Exsudierende, infizierte Wunden mit starker Geruchsentwicklung, auch bei exulzerierenden Tumoren in der Palliativmedizin.

\section{Kontraindikation}

Nicht auf trockene Wunden!

\section{Anwendungsweise}

- nicht zerschneiden (Ausnahme Carbonet)!

- Kohleseite auf die Wundfläche

- locker in tiefere Wunden einbringen/auf Wunde auflegen.

\section{Exulzerierende Metastasen:}

- Verband nicht zuschneiden

- in die Wunde einlegen

- bei geringer Exsudation Verband anfeuchten, ggf. mit einer neutralen Salbengaze unterlegen $\rightarrow$ beim Anfeuchten mit der Kanüle die Oberfläche berühren, sonst perlt die Lösung ab

- Saugkompressen dachziegelartig darüberlegen und fixieren, z. B. Schlauchverband. 


\section{Sekundärabdeckung:}

- notwendig

- Fixierung mit Schlauchverband, Klebefolie, Pflaster.

Verbandwechsel: bei fortbestehendem Wundgeruch (meist nach 2-4 Tagen). Sekundärverband abhängig von der Exsudatmenge, ggf. Saugkompressen.

\section{Kohleprodukte ohne antiseptische Wirkung}

\section{Produkte}

\section{Gelistet in den Helios-Kliniken}

Keine.

\section{Vergleichbare Produkte}

- Actisorb silberfrei (SYS)

- Askina Carbosorb (BBR)

- Carboflex (CON)

- Carbonet (S\&N)

- Nobacarbon (NOB)

- Vliwaktiv (L\&R).

\section{Kohleprodukte mit antiseptischer Wirkung}

\section{Produkte}

\section{Gelistet in den Helios-Kliniken}

Actisorb Silver 220 (SYS) $9,5 \times 6,5 \mathrm{~cm}, 10,5 \times 10,5 \mathrm{~cm}, 19,0 \times 10,5 \mathrm{~cm}$ ).

\section{Nicht gelistet}

- Nobacarbon-Ag (NOB)

- Vliwaktiv Ag Saugkompresse (L\&R)

- Vliwaktiv Ag Tamponade (L\&R). 


\section{Beschreibung}

Aktivkohlefasern imprägniert mit Silber, Polyester, Polyethylen in einer Saugkompresse (Polypropylen, Zellulose, Kleber).

\section{Empfohlene Verwendung}

Stärker kolonisierte oder infizierte Wunden (breite antimikrobielle Wirksamkeit auch gegen Candida albicans, MRSA, ESBL und VRE).

\section{Kontraindikation}

Silberunverträglichkeit.

\subsubsection{Honigpräparate}

\section{Produkte}

\section{Gelistet in den Helios-Kliniken}

- Activon - Manuka Honig Tube $25 \mathrm{~g}$ (ADV) ( $\triangleright$ Abb. 2.82)

- Actilite - nicht adhärentes Viskose-Netz, beschichtet mit $99 \%$ Manuka-Honig und $1 \%$ Manuka-Öl (ADV) $5 \times 5 \mathrm{~cm}, 10 \times 10 \mathrm{~cm}, 20 \times 30 \mathrm{~cm}$ ( $\triangleright$ Abb. 2.83).

\section{Vergleichbare Produkte}

- Activon Tulle, Algivon, Algivon Plus, Algivon Plus Ribbon (ADV)

- Medihoney Medizinischer Honig

- Medihoney Wundverbandmittel.

\section{Beschreibung}

Honig ist ein schon seit Jahrtausenden angewandtes Wundprodukt. Er besteht aus Wasser, unterschiedlichen Zuckern und Zuckerabbauprodukten wie Methylglyoxal, kurz MGO, Aminosäuren, Vitaminen, Mineralien, Enzymen etc. Insgesamt ist es im Gegensatz zu rein industriell hergestellten Produkten ein Gemisch aus einer Vielzahl von sehr unterschiedlichen Substanzen. Durch Maßnahmen wie Gammabestrahlung wird eine Keimfreiheit erzielt. 


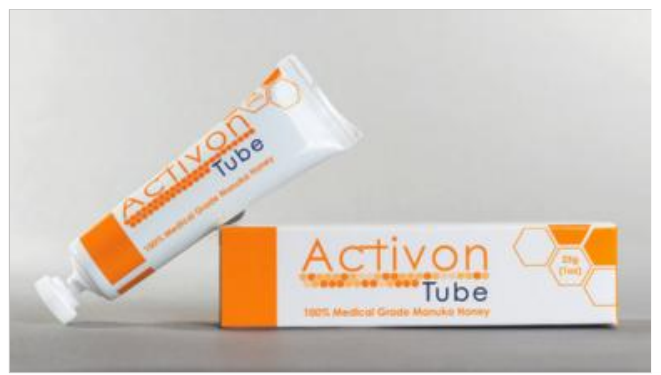

Abb. 2.82 Activon Tube.

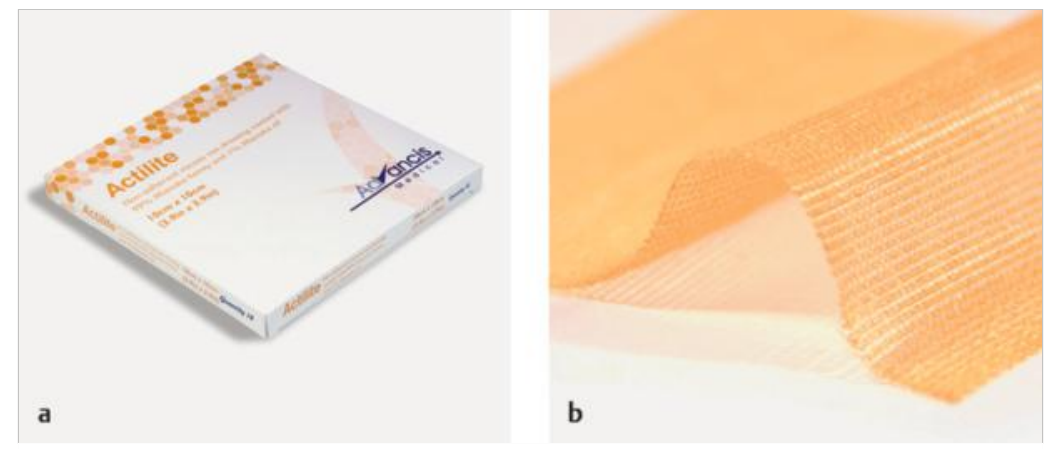

Abb.2.83 Actilite: Gaze. (Quelle: Mit freundlicher Genehmigung der Advancis medical Deutschland $\mathrm{GmbH}$ )

MGO wirkt hochgradig gegen Bakterien des Stamms Staphylococcus aureus und Escherichia coli.

Übersicht s. $>$ Abb. 2.84 . 


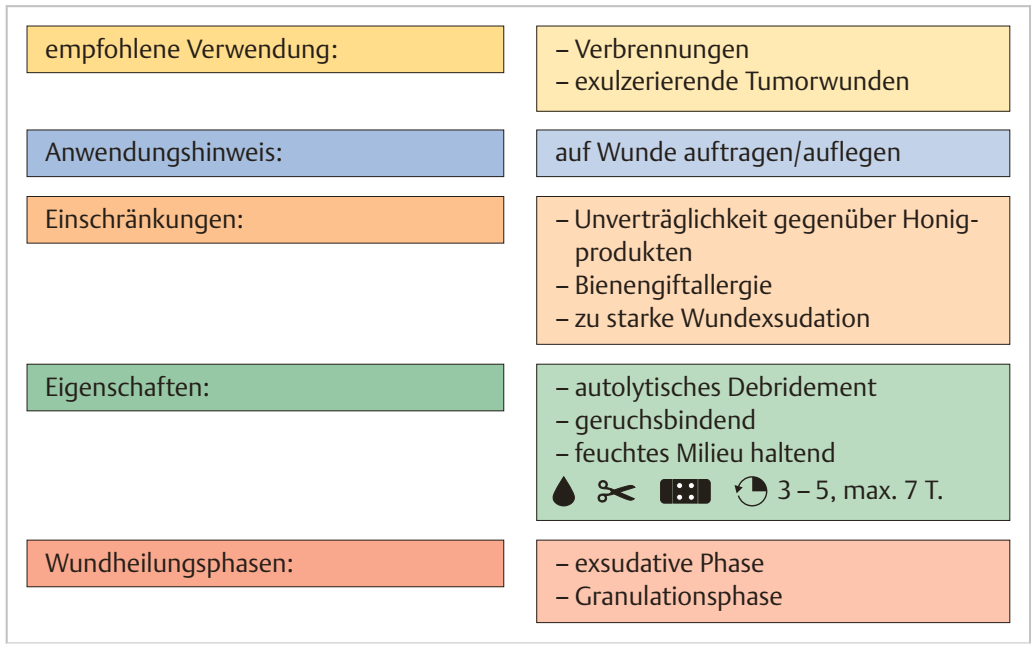

Abb. 2.84 Honigpräparate.

\section{Wirkungsweise}

Honig bewirkt ein rasches autolytisches Débridement, ist geruchsbindend und antibakteriell wirksam; die antibakterielle Wirkung soll beim Manuka-Honig besonders hoch sein. Darüber hinaus bewahrt Honig durch die osmotischen Eigenschaften ein feuchtes Wundmilieu und ist auch in der Lage, Feuchtigkeit aufzunehmen.

Produktmodifikationen: Honig als Creme, Salbe, Gel (z.B. in der Tube, - Abb. 2.85), in Gaze (z. B. Viskose), als Tüllverband etc. sowie als reines Produkt ohne Zusatzmaterialien konfektioniert.

\section{Indikation}

Verbrennungen, aber auch exulzerierende Tumorwunden.

Beworben wird die Anwendung bei praktisch allen Formen von Wunden einschließlich chronischen Wunden wie venösen Beingeschwüren, diabetische Fußwunden etc. Hier ist aber keine bessere Wirksamkeit gegenüber konventionellen Wundauflagen nachgewiesen. 


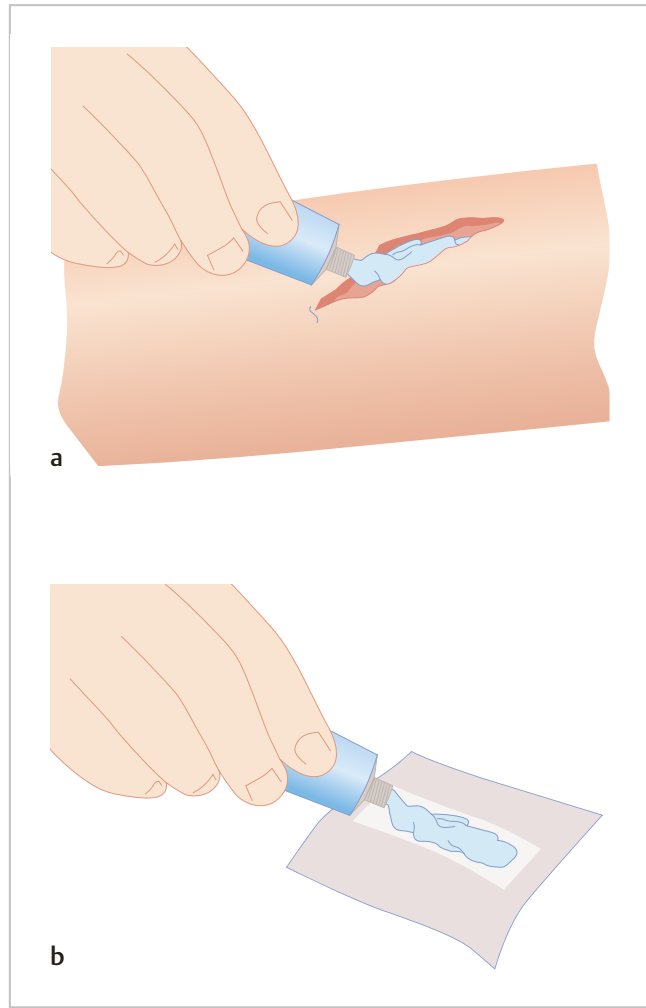

Abb. 2.85 Activon Tube:

Applikation. a, b

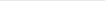

Kontraindikationen

Unverträglichkeit gegenüber Honigprodukten, Bienengiftallergie, zu starke Wund-Exsudation.

Nachteil: Kostenintensiv.

\section{Anwendungsweise}

- Die Creme 3 mm dick auftragen. 
- Die Honigprodukte werden analog vergleichbarer Produkte ohne Honig angewandt.

- Empfohlene Anwendungsdauer: bis 7 Tage.

\section{Bewertung}

Aufgrund des höheren Preises ist die Behandlung mit Honigprodukten keine Standardtherapie. Einsetzbar als Alternative zu Silberauflagen.

Eine wissenschaftliche Evidenz ist wie bei fast allen Wundprodukten nicht vorhanden! Es gibt sowohl Positiv- als auch Negativ-Empfehlungen.

\subsubsection{Hautersatzprodukte}

\section{Allgemeine Hautersatzprodukte - Wundkonditionierung vor Transplantation}

Der großflächige Verlust von Haut führt zu zahlreichen Komplikationen und erfordert häufig chirurgische Maßnahmen unter Einsatz von Hautersatzprodukten, da sonst mit einer funktionell und ästhetisch unbefriedigenden Abheilung zu rechnen ist oder die Abheilung gar nicht erzielt werden kann.

Die Wiederherstellung von Haut- und Vollhautdefekten nach z. B. Verbrennungen kann nur erfolgen, wenn sowohl die Epidermis als auch die Dermis adäquat regeneriert werden.

Hautersatzprodukte sorgen für die Schaffung eines biologischen Wundmilieus und bilden günstige Voraussetzungen für eine beschleunigte Spontanheilung, Epithelialisierung oder die Vorbereitung für eine spätere Transplantation mit günstigerem ästhetischen und funktionellen Ergebnis.

Es gibt temporäre und permanente Hautersatzprodukte.

Alle Hautersatzprodukte imitieren die eigene Haut des Patienten, damit sorgen sie im Wesentlichen für folgende Effekte:

- Verringerung des Verlustes von Flüssigkeit, Wärme, Elektrolyten, Eiweiß und Energie

- Sicherung des physiologischen Wundmilieus

- Fördern das Wachstum von Epithelzellen und Granulationsgewebe

- Schützen granulierende Wunden

- Verminderung der Schmerzen.

Temporäre Hautersatzprodukte dienen der vorübergehenden Bedeckung einer unbedingt gereinigten und sauberen Wunde bis zur Spontanheilung oder zu definitiven chirurgischen Versorgung. 
Permanente Hautersatzprodukte werden in eine gereinigte und saubere Wunde eingebracht und verbleiben dort. Sie werden integriert.

\section{Epigard}

\section{Produkt}

\section{Gelistet in den Helios-Kliniken}

Epigard (BIV) $8 \times 5 \mathrm{~cm}, 8 \times 10 \mathrm{~cm}, 8 \times 23 \mathrm{~cm}, 12 \times 30 \mathrm{~cm}, 12 \times 45 \mathrm{~cm}$

( $\triangleright$ Abb. 2.62).

\section{Vergleichbare Produkte}

Keine.

\section{Beschreibung}

Epigard besteht aus einem nichttextilen, zweischichtigen Kunststoffmaterial ohne Zusätze. Die Oberseite der offenporigen Kompresse weist eine dünne, mikroporöse luft- und wasserdampfdurchlässige Teflon-Folie auf. Exsudat-Stau wird vermieden und die Wunde wird vor von außen eindringenden Bakterien oder Flüssigkeiten geschützt. Die offene poröse Matrix auf der Unterseite ist aus elastisch-weichem Polyurethan, sie bindet Wundexsudat und Nekroseteile.

Durch Adhäsion von Thrombozyten und Exsudatgerinnung am Wundgrund erfolgt eine schnelle effektive Anhaftung. Beim ersten Verbandwechsel wird die Wunde von nekrotischem Gewebe und Wundexsudat gereinigt.

Durch den starken Granulationsreiz wird vermehrt Granulationsgewebe gebildet und Kapillaren sprossen in den Schaum ein $\rightarrow$ daher wird beim Verbandwechsel auch ein Teil des frischen Granulationsgewebes entfernt, sodass eine frische, blutende Wunde entsteht $\rightarrow$ Wundkonditionierung.

Es entsteht ein gleichmäßiger, gut vaskularisierter Wundrasen, der sekundär gedeckt werden kann ( $\triangleright$ Abb. 2.86).

Übersicht s. $\vee$ Abb. 2.87 .

\section{Indikation}

- Epigard wird überwiegend zur Wundkonditionierung vor Transplantation eingesetzt z. B. offene Frakturen mit Weichteilschäden, Weichteilschäden und großflächige Hautdefekte, kontaminierte Wundflächen

- Eignung zur Behandlung von ebenen Defektwunden ohne anschließende Hauttransplantation mit dem Ziel der spontanen Epithelisierung. 


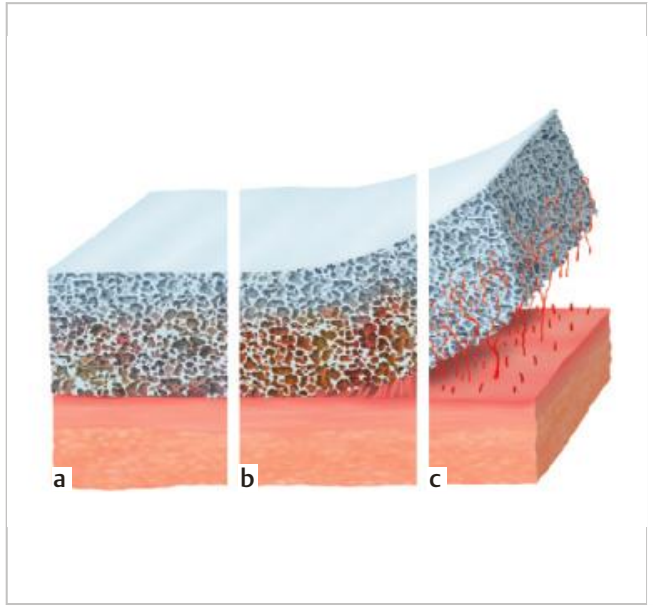

Abb. 2.86 Biovision Epigard.

a In den Hohlräumen der Polyurethanstruktur haftet das Wundexsudat.

b Nach der Wundreinigungsphase kommt es zum Einwachsen von Fibroblasten und Blutgefäßen, wodurch eine gleichmäßige und gut vaskularisierte Granulation des Wundgrundes gefördert wird.

c Beim Verbandwechsel lässt sich nekrotisches Gewebe und Wundexsudat mit Epigard abheben.
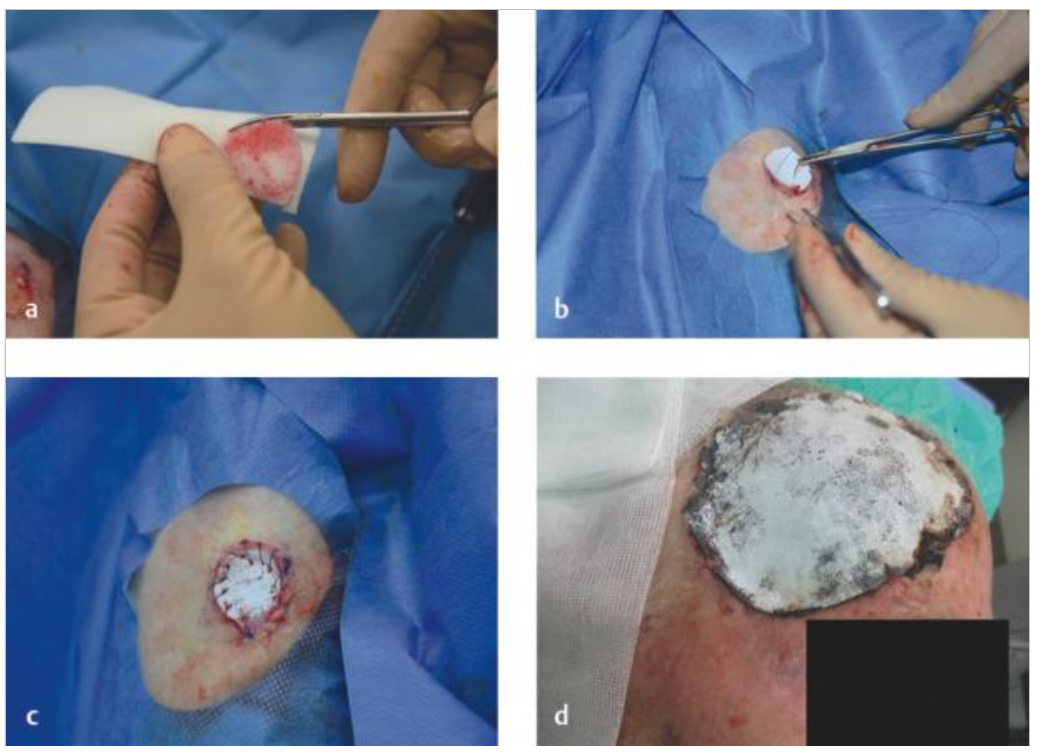

Abb. 2.87 Epigard-Anwendung.

a Abdruck und passgenaues Zuschneiden des Hautersatzes.

b Exaktes Einpassen des Hautersatzes entsprechend der Wundkontur und Fixierung.

c Fertig eingenähter Hautersatz. Es folgt der Verband unter leichter Kompression.

d Epigard nach mehreren Tagen Tragdauer. 


\section{Epigard:}

Anwendung Epigard:
- offenporiger Polyurethanschaum

- Hautersatz zur Defektauffüllung

- benötigt Untergrund mit bereits granulierendem Gewebe

- Wechsel alle 1 - 7 Tage

- bei längeren Intervallen Gefahr des Einwachsens

- abschließende Hauttransplantation erforderlich

- kostengünstig

- auf Defektgröße zuschneiden

- Fixation mit Naht, Klammern etc.

- sekundärer non-adhäsiver Schutzverband erforderlich

- bei Wundinfektion umgehende Entfernung

- regelmäßige Kontrolle bei längerem Verbleib

- mehrmaliger Wechsel möglich

- Entfernung vor Transplantation

Abb. 2.88 Epigard.

\section{Kontraindikationen}

- Wunden mit Taschenbildung

- massive Infektionen mit starker Exsudation

- eingeschränkte Blutgerinnung.

\section{Cave}

- Die Auflage darf ohne Schutz durch Hydrogele nicht auf Knorpel, Knochen, oder Sehnen direkt angewendet werden, da es sonst zur Schädigung des dünnen Häutchens über diesen Strukturen (Periost etc.) kommen kann.

- Bei trockenen Wunden besteht bei Anwendung Austrocknungsgefahr und somit die Gefahr der Nekrosenbildung! Dann mit Hydrogel unterlegen! 
- Des Weiteren sind Schmerzen beim Verbandwechsel (unerwünscht) sowie Verletzungen am Granulationsgewebe (zur Konditionierung eines blutenden Untergrundes erwünscht) aufgrund der Struktur des offenporigen Schaumverbandes möglich.

- Verbandwechsel bei Anzeichen von Irritation, Infektion oder Flüssigkeitsansammlung.

- Keine gleichzeitige Anwendung mit Creme oder Salbe.

\section{Anwendungsweise}

- sorgfältiges Wunddébridement zur Schaffung übersichtlicher Wundverhältnisse

- exaktes Anpassen des Hautersatzes und genaues Zuschneiden an die Wundkontur

- Verband mit leichter Kompression.

Verbandwechsel: Der Verbandwechsel sollte nach mehreren Tagen erfolgen. Bei zu langer Verbandliegedauer kann das Granulationsgewebe in den Schaum einwachsen.

Wechsel nach Herstellerangaben alle 24-48 Stunden, Kontrolle der Wunde, steriler Verband. Wiederholte Anwendung von Epigard bis zur Erzielung eines transplantationsreifen Granulationsrasens.

Definitiver Wundverschluss durch Spalthauttransplantation oder Spontanepithelisierung, ggf. auch verzögerte Primärnaht.

\section{Xenoderm}

\section{Produkte}

\section{Gelistet in den Helios-Kliniken}

Xenoderm (MBP) $50 \times 50 \times 0,3 \mathrm{~mm}, 100 \times 100 \times 0,3 \mathrm{~mm}, 100 \times 200 \times 0,3 \mathrm{~mm}$, $100 \times 300 \times 0,3 \mathrm{~mm}, 100 \times 400 \times 0,3 \mathrm{~mm}(\triangleright$ Abb. 2.89). 

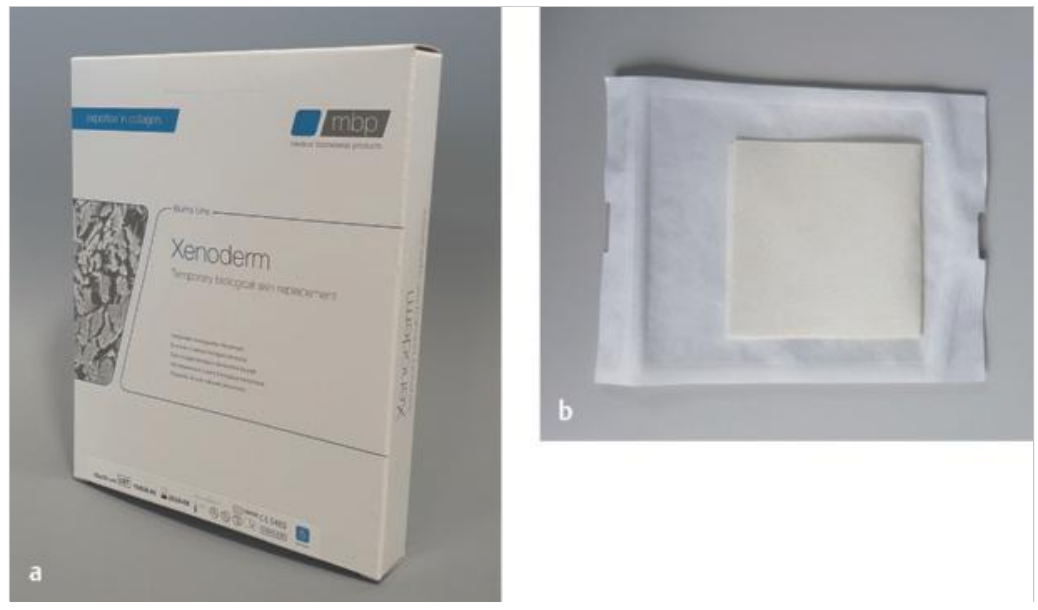

Abb. 2.89 Xenoderm. a, b (Quelle: Mit freundlicher Genehmigung der MBP GmbH)

\section{Beschreibung}

Xenoderm ist ein biologischer temporärer Hautersatz, der aus Spalthaut gewonnen wird. Es handelt sich um eine azelluläre gefriergetrocknete, porcine Kollagenmatrix. Sie wirkt klinisch nicht antigen und ist gut anmodellierbar. Es erlaubt durch seine Transparenz die Beobachtung des Wundgrundes, der Patient kann sich gut bewegen, was die frühe Mobilisation und Rehabilitation fördert ( $\vee$ Abb. 2.90).

Übersicht s. $>$ Abb. 2.91 . 


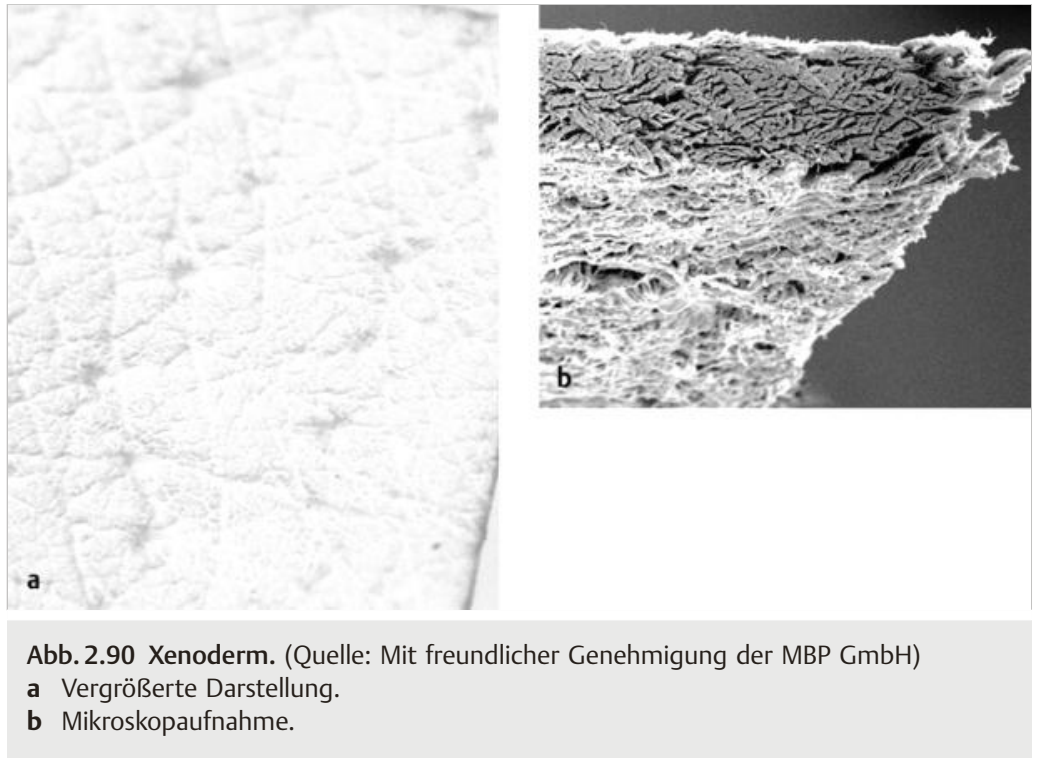

\section{Indikation}

Zur temporären Abdeckung nach ausreichendem chirurgischen Débridement von Verbrennungen (Grad 2a, 2b, 3), traumatischen Wunden und allen oberflächlichen und tiefen Wunden; Einsatz auch bei verzögerter Abheilung nach Débridement, zur Abdeckung autologer Transplantate.

\section{Kontraindikationen}

- Verbrennungen 3. Grades vor der Entfernung des Schorfs

- massiv infizierte Wunden vor entsprechender Reinigung (Débridement)

- Patienten mit Sensibilisierung für Materialien porcinen Ursprungs.

\section{Anwendungsweise}

- Sterile Entnahme und anschließende Rehydration für mind. 2 Minuten in $0,9 \% \mathrm{NaCl}$-Lösung $\rightarrow$ wird durchsichtig.

- Aufbringen des zugeschnittenen Produktes auf die debridierte und antiseptisch vorbehandelte Wunde. 
Xenoderm:

Anwendungshinweis:
- aus Spalthaut gewonnene porcine, azelluläre Kollagenmatrix

- ein-/mehrmalige Aufbringung

- kostengünstig

- Verband sollte in $\mathrm{NaCl}$ für 2 min eingeweicht werden (wird durchsichtig)

- auf Defektgröße zuschneiden

- Cave: Mazerationsrisiko an lasttragenden Arealen

- Fixierung mittels Kompressionsverband etc.

- sekundärer non-adhäsiver Schutzverband erforderlich

- regelmäßige Kontrolle bei längerem Verbleib

- bei Wundinfektion umgehende Entfernung

- Selbstablösung bei Epithelisierung, sonst Entfernung nach 8-10 Tagen

Abb. 2.91 Xenoderm.

- Einpassen, fixieren mit Naht, etc.

- Auf reepithelisierten Wunden (Verbrennung IIa) löst sich Xenoderm mit dem Grad der Epithelisierung, ansonsten muss es spätestens nach $10 \mathrm{Ta}-$ gen entfernt oder gewechselt werden.

- Das Ablösen wird durch Befeuchten mit 0,9\% NaCl-Lösung erleichtert.

- Bei fehlender Reepithelisation ist ein sekundärer Epithelverschluss durch Spalthaut-Transplantat etc. erforderlich.

- Es ist auf festen Kontakt zum Wundgrund (keine Luftblasen) und ausreichende Fixierung durch einen Sekundärverband zu achten.

\section{Cave}

- Kein Einsatz ohne Wunddébridement!

- Nicht auf lasttragenden Bereichen des Körpers (Mazerationsrisiko) anwenden. 


\section{Verbandwechsel:}

- Auf einen regelmäßigen Wechsel des Sekundärverbandes ist zu achten. Die Verbleibdauer auf der Wunde ist variabel und wird vom Wundheilungsverlauf bestimmt (max. 10 Tage).

- Auf Wunden 2. Grades kann einmal haftendes Xenoderm belassen werden, bis es sich beim Eintritt der Reepithelisierung ablöst.

- Fixation durch Mullbinden oder andere geeignete Verbandsmaterialien.

\section{Merke}

Aufgrund des porcinen Ursprungs in der Herstellung von Xenoderm müssen Patienten mit einem muslimischen Hintergrund diesbezüglich aufgeklärt werden.

\section{Integra}

\section{Produkte}

\section{Gelistet in den Helios-Kliniken}

- IDRT-TS (INT) $5 \times 5 \mathrm{~cm}, 10 \times 12,5 \mathrm{~cm}, 10 \times 25 \mathrm{~cm}, 20 \times 25 \mathrm{~cm}$

- IDRT-Single Layer (INT) $5 \times 5 \mathrm{~cm}, 10 \times 12,5 \mathrm{~cm}, 10 \times 25 \mathrm{~cm}, 20 \times 25 \mathrm{~cm}$

- Integra Flowable Wound Matrix Kit (INT) 3 ml.

\section{Beschreibung}

Integra gibt es als Single Layer und Bi-Layer mit PU Folie beschichtet.

Integra Bi-Layer ist ein zweischichtiger, alloplastischer Hautersatz. Er besteht zu $90 \%$ aus einer bovinen dreidimensionalen Porenmatrix aus Typ-1-Kollagen und $10 \%$ anteilig Chondroitin-6-Sulfat sowie einer Silikonschicht und dient als permanenter Dermisersatz. Das Kollagen stammt von BSE-freien australischen Rindern.

Integra wird auf ein gut durchblutetes Wundbett aufgebracht und es sprossen Bindegewebszellen des Empfängers in die Matrix ein, innerhalb von 14-21 Tagen ist die Matrix dann vaskularisiert und in Neodermis umgebaut.

Die schützende Silikonfolie kann entfernt werden und durch eine dünne Spalthaut oder Keratinozytensheets ersetzt werden.

Bei Auflage auf schwach vaskularisierten Arealen wie Schädelknochen ohne Periost kann dieser Prozess auch bis zu 40 Tagen oder mehr dauern.

Übersicht s. > Abb. 2.92. 


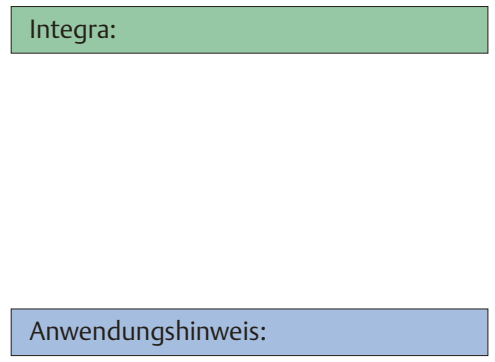

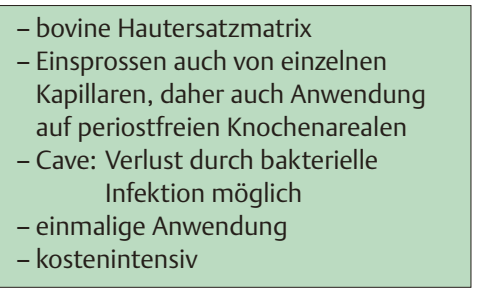

- auf Defektgröße zuschneiden

- Fixation mit Naht, Klammern etc.

- sekundärer non-adhäsiver Schutzverband erforderlich

- mehrwöchige Liegezeit $\rightarrow$ regelmäßige Kontrollen

- bei Wundinfektion umgehende Entfernung bzw. Evakuierung

- bei Bi-Layer $\rightarrow$ Folie vor Transplantation entfernen

Abb. 2.92 Integra.

\section{Indikation}

Integra kann zur Abdeckung von traumatischen Wunden und allen oberflächlichen und tiefen Wunden, schwer heilenden Wunden mit freiliegenden Knochenanteilen, auch Verbrennungswunden (Grad 2-3) benutzt werden.

\section{Kontraindikationen}

- Einsatz vor Wunddébridement, auch bei massiv infizierten Wunden

- Patienten mit Sensibilisierung für Materialien bovinen Ursprungs oder Silikon

- druckbelastete Körperbereiche.

\section{Anwendungsweise}

- Wunddébridement mit guter Blutstillung.

- Sterile Entnahme und anschließendes Abspülen mit Ringer-/NaCl-Lösung.

- Aufbringen des zugeschnittenen Produktes auf die debridierte und antiseptisch vorbehandelte Wunde. 
- Einpassen, fixieren mit Naht, etc.

- Es ist auf festen Kontakt zum Wundgrund (keine Luftblasen) und ausreichende Fixierung durch einen Sekundärverband zu achten-

- Es ist ein sekundärer Epithelverschluss durch Spalthaut-Transplantat etc. erforderlich (einzeitig beim Mono-Layer, mehrzeitig beim Bi-Layer).

\section{Verbandwechsel:}

Auf einen regelmäßigen Wechsel des Sekundärverbandes ist zu achten. Die Verbleibdauer auf der Wunde ist variabel und wird vom Wundheilungsverlauf bestimmt (kann bis zu 10 Wochen belassen werden).

Anwendung von Integra s. > Abb. 2.93.
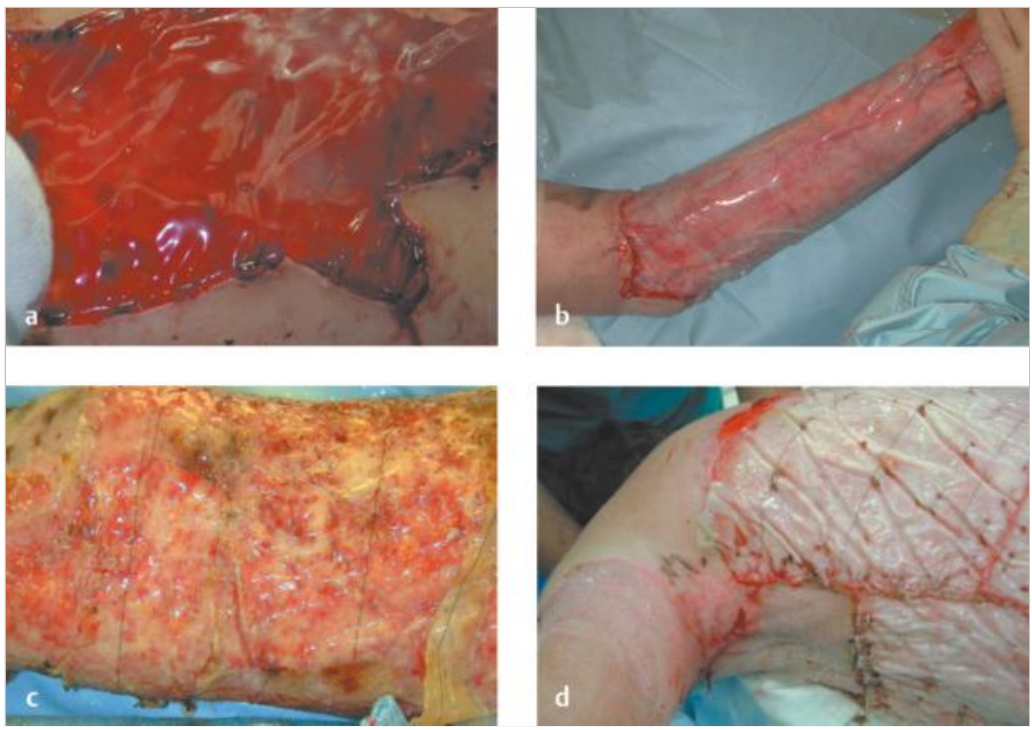

Abb. 2.93 Integra: Anwendung. (Quelle: Mit freundlicher Genehmigung der Integra LifeSciences Corporation, Plainsboro, New Jersey, USA)

a Tag 0: Applikation Rotfärbung der Matrix.

b Tag 7: Farbwechsel von ROT zu ROSA. Die Neodermis-Bildung ist in vollem Gange. Zelluläre Infiltration ändert die Matrixfarbe.

c Tag 14: Farbe: ORANGE-PEACH. Vaskularisation ist im Gange.

d Tag 21: Farbe VANILLE. Vor der Silikonentfernung. Die Neodermis ist ausgereift und bereit für ein epidermales Autotransplantat. 


\section{MatriDerm}

\section{Produkte}

\section{Gelistet in den Helios-Kliniken}

- MatriDerm A8 (SOR) $52 \times 74 \times 1 \mathrm{~mm}$

- MatriDerm A6 (SOR) $105 \times 148 \times 1 \mathrm{~mm}$

- MatriDerm A4 (SOR) $210 \times 297 \times 1 \mathrm{~mm}$ ( $\vee$ Abb. 2.94).

\section{Beschreibung}

MatriDerm ( $\vee$ Abb. 2.95) ist eine bovine Collagen-Elastin-Matrix, die nach Hautverlust zur Regeneration und Rekonstruktion der Haut eingesetzt wird. Es wurde nach dem Vorbild der Dermis als dreidimensionale Matrix aus Collagen und Elastin entwickelt.

Die Matrix bietet nach Transplantation körpereigener Zellen das Grundgerüst für die geordnete Neubildung der Dermis. Als Leitschiene für Zellen und Gefäße dient die Collagenmatrix. Das Elastin verbessert die Stabilität und Elastizität des neuen Gewebes.

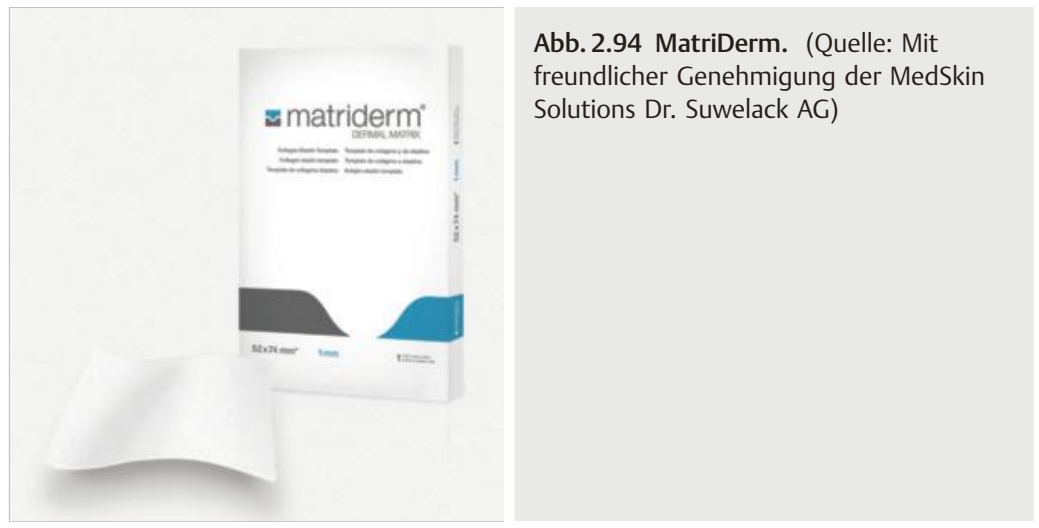




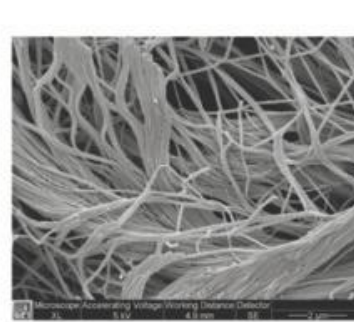

Human dermis

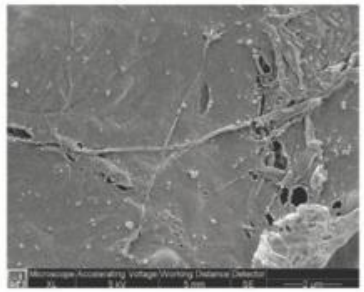

Other processed dermal matrix

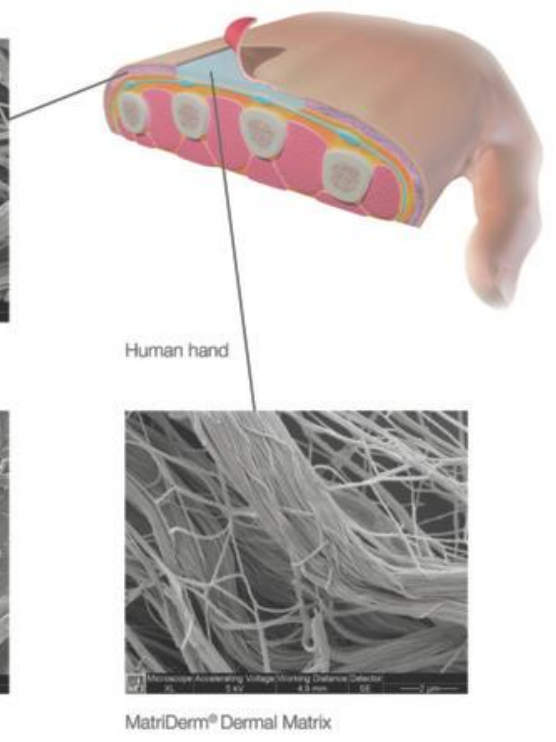

Abb. 2.95 MatriDerm: Struktur. (Quelle: Mit freundlicher Genehmigung der MedSkin Solutions Dr. Suwelack AG)

Fibroblasten produzieren ihre eigene extrazelluläre Matrix und MatriDerm wird resorbiert. Aufgrund seiner hämostyptischen Eigenschaft wird die Entstehung eines Hämatoms unter dem Spalthauttransplantat reduziert.

MatriDerm passt sich in nassem Zustand der Oberfläche problemlos an und ist überdurchschnittlich reißfest.

Die rekonstruierte Haut (MatriDerm als Dermis-Ersatz und darüber Spalthaut) ist früh nach der Operation elastischer und mechanisch belastbarer als alleinige Spalthaut-Transplantate und neigt weniger stark zur Schrumpfung. Übersicht s. - Abb. 2.96.

\section{Indikation}

- Tiefe dermale Defekte.

- Chronische Wunden. 


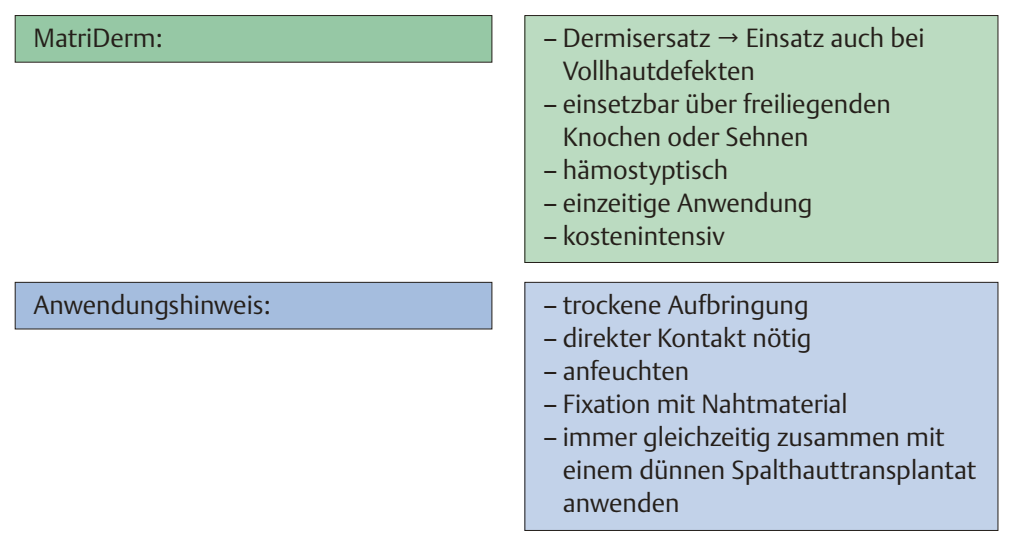

Abb. 2.96 MatriDerm.

- Zum Dermisaufbau bei Vollhautdefekten in Kombination mit autologen Spalthauttransplantaten. Um alle Elemente der normalen Haut rekonstruieren zu können, wird die Matrix mit dünner Spalthaut gedeckt.

- Temporäre Gewebetrennung unter intakter Haut.

\section{Kontraindikation}

Infizierte/nicht gereinigte Wunden.

\section{Anwendungsweise}

- Débridement der Wunde und Herstellung eines sauberen und gut durchbluteten Wundbettes frei von jodhaltigen Antiseptika sowie von Wirkstoffen für ein enzymatisches Débridement

- trockene Aufbringung auf den vorbereiteten Wundgrund

- mehrere MatriDerm-Matrizes sollen nicht überlappend, sondern bündig eingelegt werden

- Anfeuchten mit 0,9\% NaCl, dann zuschneiden auf die Wundgröße (1-2 mm überlappend) $\rightarrow$ Wundbett komplett abdecken und auf guten Wundrandkontakt achten 
- auf direkten Kontakt mit dem Wundbett achten, Luftblasen ausstreichen

- MatriDerm $1 \mathrm{~mm}$ wird immer gleichzeitig zusammen mit einem dünnen ungemeshten Spalthauttransplantat angewendet; optional Inzisionen zur Drainage von Wundexsudat; Anwendung von gemeshtem Transplantat $1: 1,5$ empfohlen

- direkter Kontakt zwischen MatriDerm und dem Hauttransplantat

- Fixierung von beidem mit Nähten, Klammern oder Fibrinkleber; bei Vakuumverband keine zusätzliche Fixation nötig

- Auflegen einer Fettgaze und Polsterung mit z. B. Kompressen, NPWT möglich.

Verbandwechsel: In der Regel wird bei MatriDerm einzeitig die Haut transplantiert. In diesem Fall erfolgt der erste Verbandswechsel routinemäßig am 5.-7. postop. Tag, bei zuvor stark kontaminierten Wunden schon am 3. Tag.

\section{Spezielle Hautersatzprodukte (Verbrennung)}

\section{Biobrane}

\section{Produkte}

\section{Gelistet in den Helios-Kliniken}

Biobrane (S\&N) $13 \times 13 \mathrm{~cm}$, Handschuhe Päd, Handschuh M ( $\vee$ Abb. 2.97).
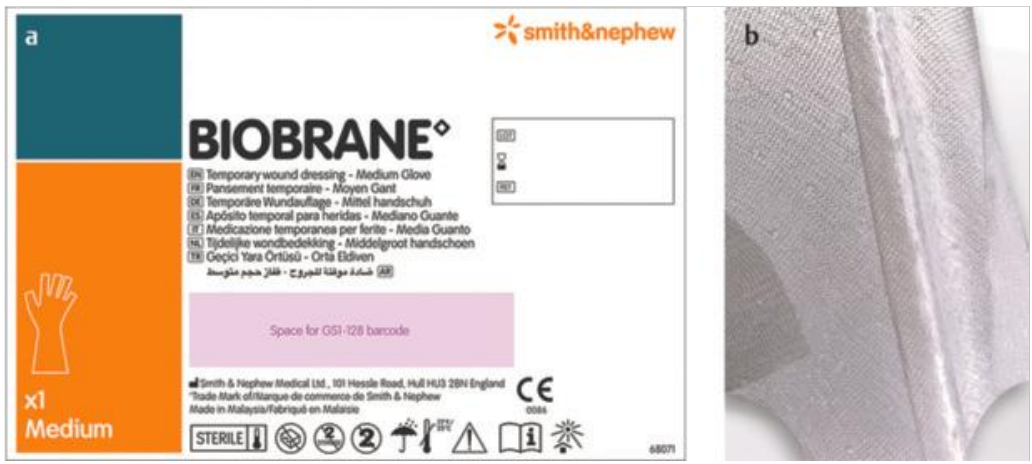

Abb. 2.97 Biobrane. a, b (Quelle: Mit freundlicher Genehmigung der Smith \& Nephew $\mathrm{GmbH})$ 


\section{Beschreibung}

Biobrane ist ein temporärer alloplastischer, nicht resorbierbarer Hautersatz aus einer semipermeablen Silikonmembran mit 3-D Nylongewebe besetzt mit porcinem Kollagen und Polygluconat ( $\triangleright$ Abb. 2.98, $\triangleright$ Abb. 2.99). Der Hautersatz haftet biologisch durch Bindung des Nylongewebes mit Hilfe von Blut und Serum auf der Wunde ( $\triangleright$ Abb. 4.15).
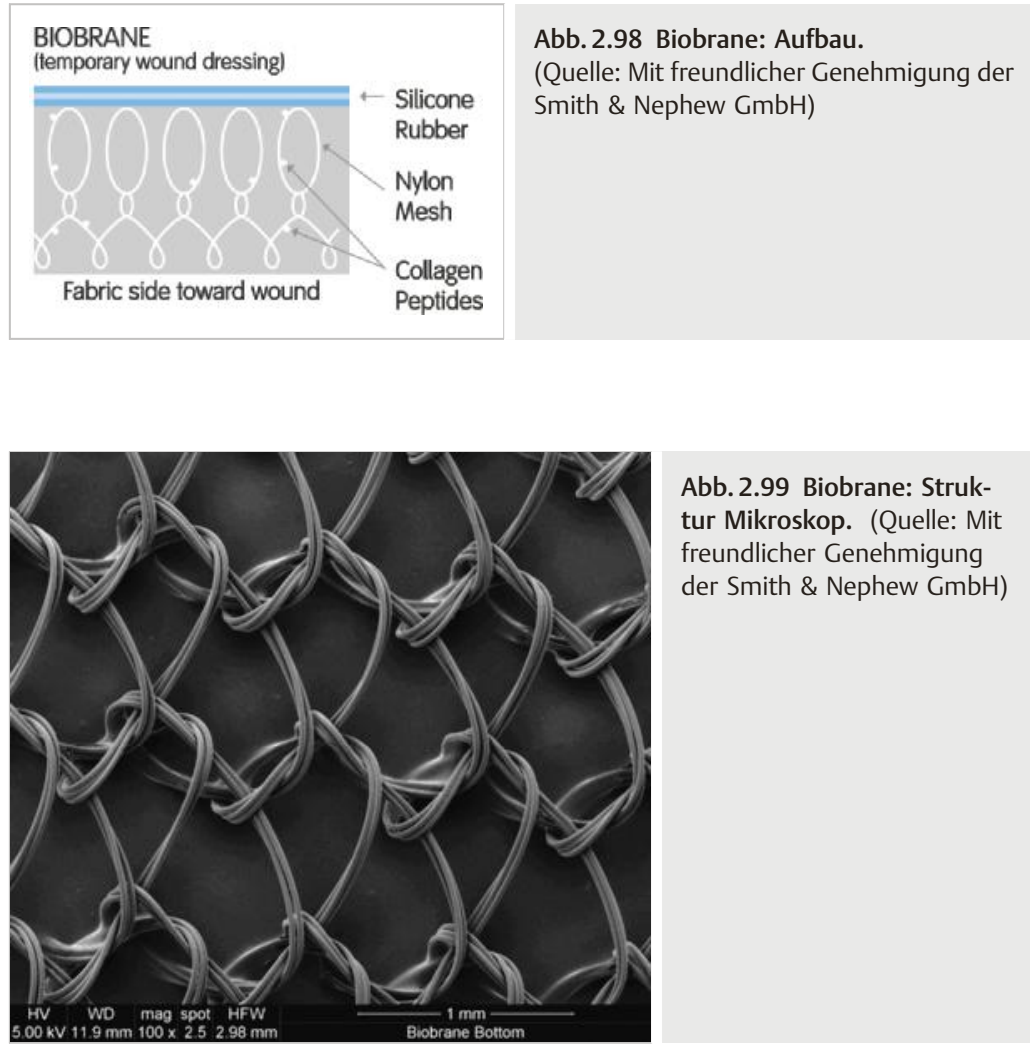

Abb. 2.99 Biobrane: Struktur Mikroskop. (Quelle: Mit freundlicher Genehmigung der Smith \& Nephew GmbH) 


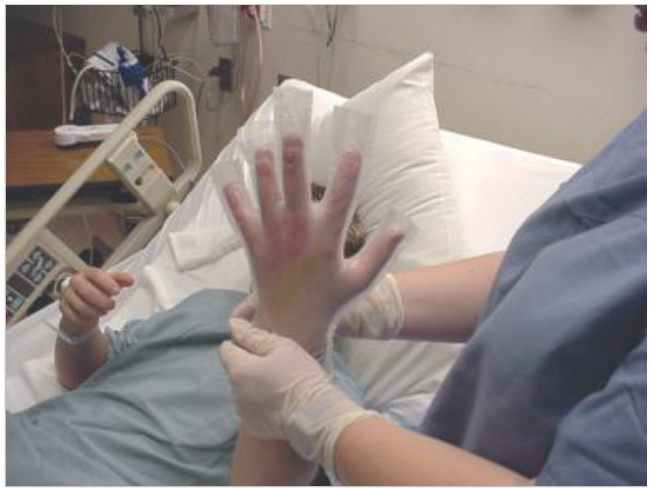

Abb. 2.100 Biobrane: Glove. (Quelle: Mit freundlicher Genehmigung der Smith \& Nephew GmbH)

Biobrane kann auf der Wunde verbleiben, bis die Oberfläche neu epithelisiert ist (Transplantation nicht zwingend erforderlich!). Öffnungen in Biobrane erlauben den Austritt von Exsudat durch die Silikonmembran. Die Mobilität von Patienten wird durch die Flexibilität von Biobrane erhöht.

Für Verbrennungen der Hände gibt es spezielle Handschuhe in vier Größen, die exakt angepasst werden können ( $\triangleright$ Abb. 2.100).

Biobrane ist sehr gut auch an Körperteile wie Hände, Füße und Gesicht anpassbar.

Die Gliedmaßen können darunter ohne Verlust der Wundabdeckung mobilisiert werden.

Übersicht s. > Abb. 2.101. 


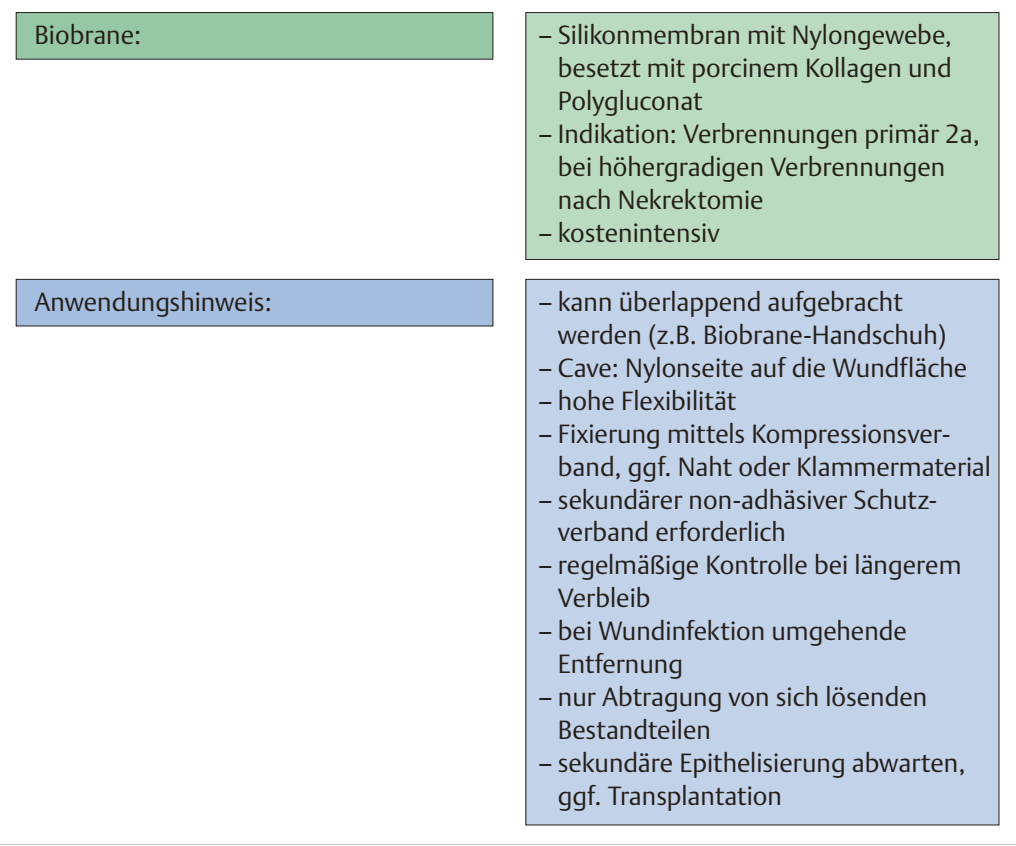

Abb. 2.101 Biobrane.

\section{Indikation}

Verbrennungen Grad 2a.

\section{Kontraindikationen}

- Verbrennung ab Grad 2b.

- eröffnete Blasen > 1 h ohne sterile Abdeckung

- thermische Verletzungen, Stromverletzungen, Verätzungen durch Säuren und Laugen Grad 1 und (3-) 4

- ältere Verletzungen mit klinisch bereits infizierten Wunden. 


\section{Merke}

Aufgrund der temporären Abdeckung der Wundflächen sind Verbandwechsel des Sekundärverbandes schmerzarm bis schmerzfrei möglich.

Die Anwendung von Biobrane-Handschuhen erleichtert die Versorgung komplexer Verletzungen an der Hand.

\section{Anwendungsweise}

- Die Brandblasen unter sterilen Bedingungen vollständig abtragen.

- Das verletzte Areal rasieren.

- Aufbringen unter sterilen Bedingungen mit der korrekten Seite zur Wunde liegend.

- Fixierung mittels Kompressionsverband, ggf. Naht oder Klammermaterial.

- Die Entfernung des Klammer- bzw. Nahtmaterials kann nach 4 Tagen, nach Erreichen einer festen Verbindung zwischen Biobrane und dem Wundgrund, erfolgen.

- Das Abtragen von Biobrane selbst erfolgt tangential von der Seite der Verletzung unter steter Entfernung der sich lösenden Areale.

- Zum Schutz des Wundareals vor mechanischer Alteration sollte eine einlagige Abdeckung mit einem fetthaltigen Wunddistanzgitter erfolgen.

- Darüber kann ein Mullverband gegen mechanische Alteration oder Verschmutzung schützen.

- Dieser Verband kann schmerzfrei gewechselt werden.

- Das Produkt und das fetthaltige Wunddistanzgitter kann bis zur vollständigen Epithelisierung der Wunde belassen werden.

- Bei Kindern ist in der Regel die Anlage in Sedierung sinnvoll.

\section{Verbandwechsel:}

- Normalerweise genügt ein Verbandwechsel des Sekundärverbandes alle 2-3 Tage.

- Im Rahmen jedes Verbandwechsels können die lockeren Seitenränder der Produkte abgetragen werden. Nach spätestens 14 Tagen kann die restliche Wundauflage entfernt werden. Verbliebene Wunden bedürfen in der Regel einer chirurgischen Sanierung. 


\section{Merke}

- Im Rahmen des Verbandwechsels sollte ein fetthaltiges Wunddistanzgitter mit dem darunter befindlichen Hautersatz auf der Wunde verbleiben. Ein vorsichtiges Ablösen des Überverbandes ist zwingend notwendig, um einen Verlust des Hautersatzes zu vermeiden. Im Rahmen des Verbandwechsels sollte keine Feuchtigkeit auf den Verband aufgebracht werden.

- Aufgrund des porcinen Ursprungs in der Herstellung von Biobrane müssen Patienten mit einem muslimischen Hintergrund diesbezüglich aufgeklärt werden.

\section{Cave}

Bei Anzeichen einer Wundinfektion ist der Hautersatz umgehend zu entfernen.

\section{Suprathel}

\section{Produkte}

\section{Gelistet in den Helios-Kliniken}

Suprathel (POL) $5 \times 5 \mathrm{~cm}, 9 \times 10 \mathrm{~cm}, 10 \times 18 \mathrm{~cm}, 18 \times 23 \mathrm{~cm}(\triangleright$ Abb. 2.102).

\section{Beschreibung}

Suprathel ist ein alloplastischer resorbierbarer Hautersatz aus einer PolylactatMembran (Polymilchsäure); Struktur s. \Abb. 2.103. Einsatz bei epidermaler und dermaler Wunde.

Nach Aufbringung wird die Membran transparent und ermöglicht so die Beobachtung des Heilungsfortschritts. Passt sich gut an Körperteile an.

Einmalige Aufbringung. Eine operative Entfernung ist nicht erforderlich, da Suprathel innerhalb von 4-6 Wochen degradiert wird. Suprathel löst sich an wiederhergestellten Hautarealen ab.

Übersicht s. \ Abb. 2.104. 


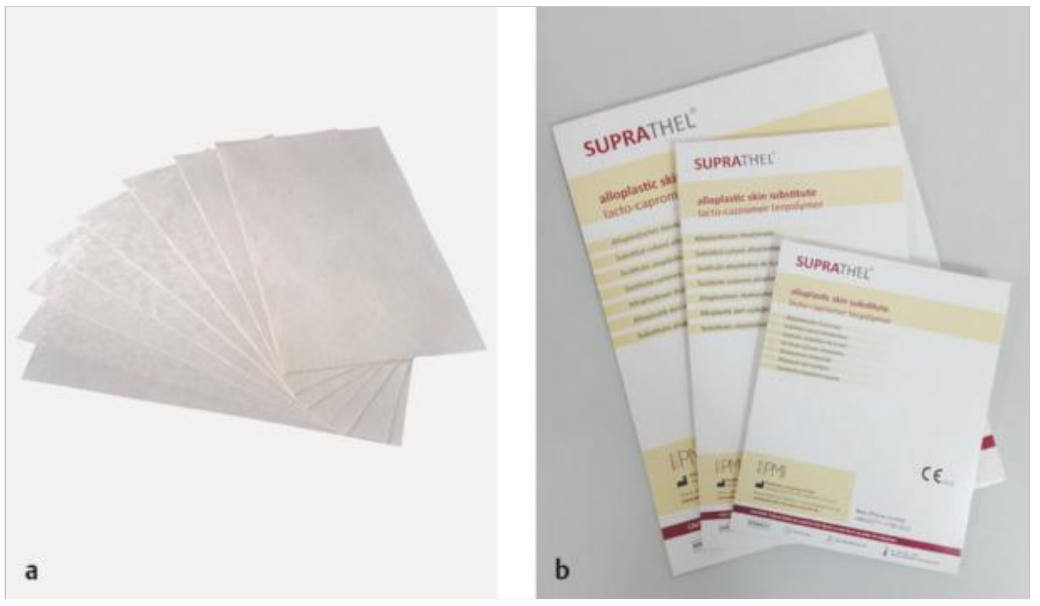

Abb. 2.102 Suprathel. a, b (Quelle: Mit freundlicher Genehmigung der PolyMedics Innovations $\mathrm{GmbH}$ )

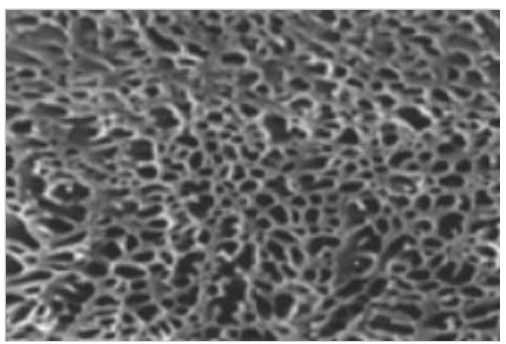

Abb. 2.103 Suprathel: Struktur (Mikroskop). (Quelle: Mit freundlicher Genehmigung der PolyMedics Innovations $\mathrm{GmbH}$ )

\section{Indikation}

- epidermale und dermale Wunden

- Verbrennungen (Grade 2a-2b, auch bei partiellen Grad-3-Anteilen)

- Spalthautentnahmestellen

- Schürfwunden. 
Suprathel:

Anwendungshinweis:
- alloplastisch aus Polylactatmembran

- nach Aufbringung wird die Membran transparent und ermöglicht so die Beobachtung des Heilungsfortschritts

- Degradation nach 4-6 Wochen

- Ablösung an wiederhergestellten Hautarealen

- einmalige Anwendung

- sehr kostenintensiv

- auf Defektgröße zuschneiden

- Fixation mit Naht, Klammern etc.

- Fixierung mittels Kompressionsverband, ggf. Naht oder Klammermaterial

- sekundärer non-adhäsiver Schutzverband erforderlich

- regelmäßige Kontrolle bei längerem Verbleib

- bei Wundinfektion umgehende Entfernung

- nur Abtragung von sich lösenden Bestandteilen

- sekundäre Epithelisierung abwarten, ggf. Transplantation

Abb. 2.104 Suprathel.

\section{Kontraindikationen}

- eröffnete Blasen $>1 \mathrm{~h}$ ohne sterile Abdeckung

- infizierte Verbrennungen

- Verbrennungen > Grad 2b, Ulzera o. Ä.

- blutende Wunden

- ältere Verletzungen mit klinisch bereits infizierten Wunden. 


\section{Merke}

\section{Anwendungsweise}

- Abtragung der Brandblasen und Säuberung der Wunde (steril).

- Rasur um das Verbrennungsareal.

- Aufbringen von Suprathel unter sterilen Bedingungen.

- Entsprechend der Form und Größe der Wunde wird der Verband geformt oder zugeschnitten und locker, blasenfrei und nicht überlappend aufgelegt.

- Der Verband haftet selbstständig.

- Eine zusätzliche Fixierung z. B. mittels Einzelknopfnaht ist in der Regel nicht notwendig.

- Zum Schutz des Wundareals vor mechanischer Alteration muss eine einlagige Abdeckung mit einem fetthaltigen Wunddistanzgitter erfolgen.

- Darüber kann ein Mullverband gegen mechanische Alteration oder Verschmutzung schützen. Dieser Verband kann schmerzfrei gewechselt werden.

- Produkt und fetthaltiges Wunddistanzgitter können bis zur Abheilung der Wunde belassen werden.

Anwendung von Suprathel s. - Abb. 2.105, > Abb. 2.106.

Verbandwechsel: Normalerweise genügt ein Verbandwechsel des Sekundärverbandes alle 2-3 Tage. Im Rahmen jedes Verbandwechsels können die lockeren Seitenränder der Produkte abgetragen werden. Nach spätestens 14 Tagen kann die restliche Wundauflage entfernt werden. Verbliebene Wunden bedürfen in der Regel einer chirurgischen Sanierung.

\section{Merke}

Im Rahmen des Verbandwechsels sollte ein fetthaltiges Wunddistanzgitter mit dem darunter befindlichen Hautersatz auf der Wunde verbleiben. Ein vorsichtiges Ablösen des Überverbandes ist zwingend notwendig, um einen Verlust des Hautersatzes zu vermeiden. Im Rahmen des Verbandwechsels sollte keine Feuchtigkeit auf den Verband aufgebracht werden. 

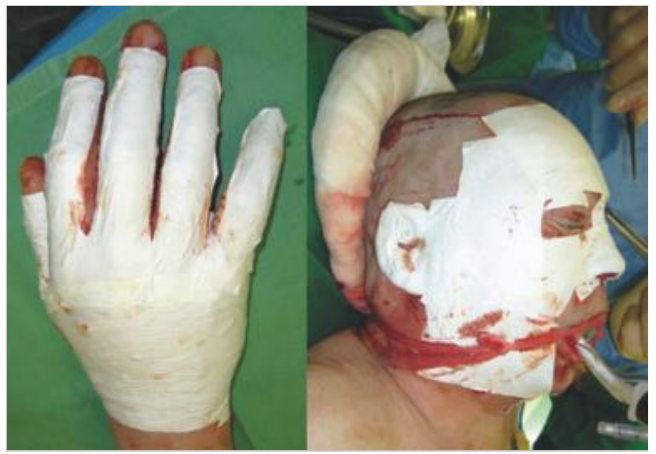

Abb. 2.105 Suprathel:

Anwendung im Gesicht und an der Hand. (Quelle: Mit freundlicher Genehmigung der PolyMedics Innovations $\mathrm{GmbH}$ )
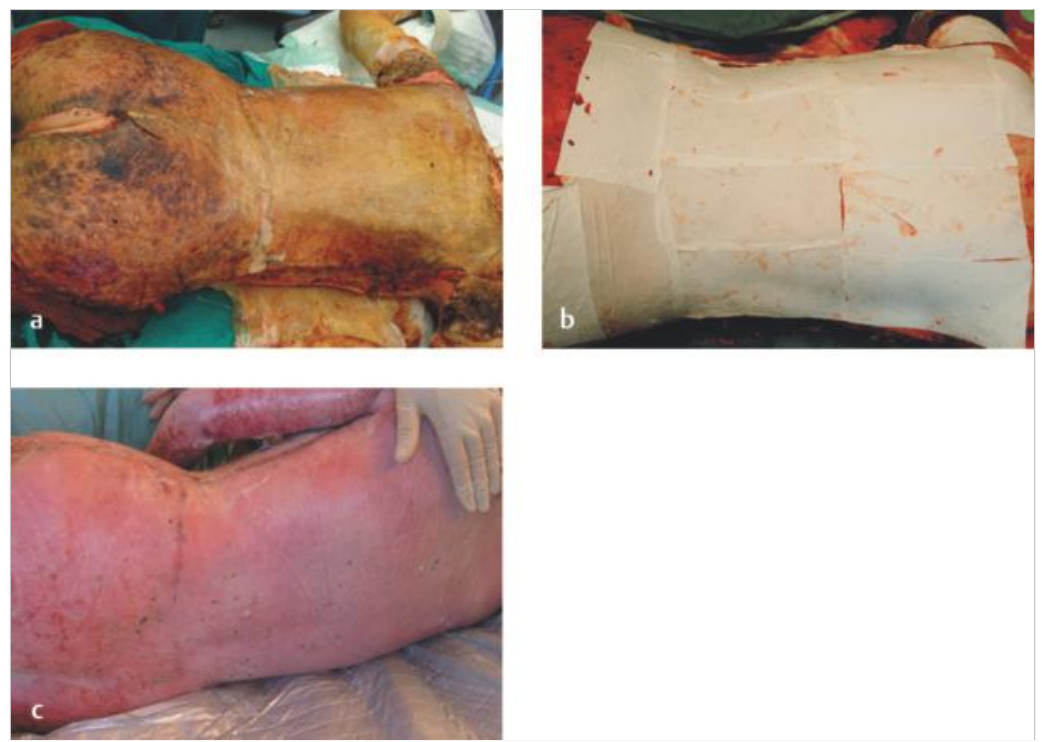

Abb.2.106 Suprathel: Anwendung am Rücken. a-c (Quelle: Mit freundlicher Genehmigung der PolyMedics Innovations $\mathrm{GmbH}$ )

\section{Cave}

Bei Anzeichen einer Wundinfektion ist der Hautersatz umgehend zu entfernen 


\section{Kerecis Omega3}

\section{Produkte}

\section{Gelistet in den Helios-Kliniken}

Kerecis (KER) $3 \times 3,5 \mathrm{~cm}, 3 \times 7 \mathrm{~cm}, 7 \times 10 \mathrm{~cm}$ ( $\triangleright$ Abb. 2.107).

\section{Beschreibung}

Kerecis Omega3 Wound ist eine azelluläre, biologisch aktive Gewebematrix (Schema s. - Abb. 2.108) aus intakter Fischhaut zum dermalen Hautersatz sowie zum Einsatz an anderen Gewebestrukturen schwer oder nicht heilender Wunden. Natürlich vorhandene Omega-3-Fettsäuren fördern den Heilungsprozess durch antiinflammatorische Wirkung.

Durch Anwendung können Stammzellen (Progenitorzellen) die Kerecis Omega3-Matrix besiedeln und sich darin ausbreiten. Dadurch wird das Remodelling induziert. Das durch die azelluläre Matrix vorgegebene Baugerüst wird durch die Stammzellen und die sich vermehrenden Zellen sowohl um-, als auch teilweise abgebaut, wobei daraus neues körpereigenes Gewebe entsteht.

Die bradytrophe Kollagen-Matrix ermöglicht darüberhinaus, freiliegende Sehnen und Knochen zu decken und damit in ihrer Funktion zu erhalten.

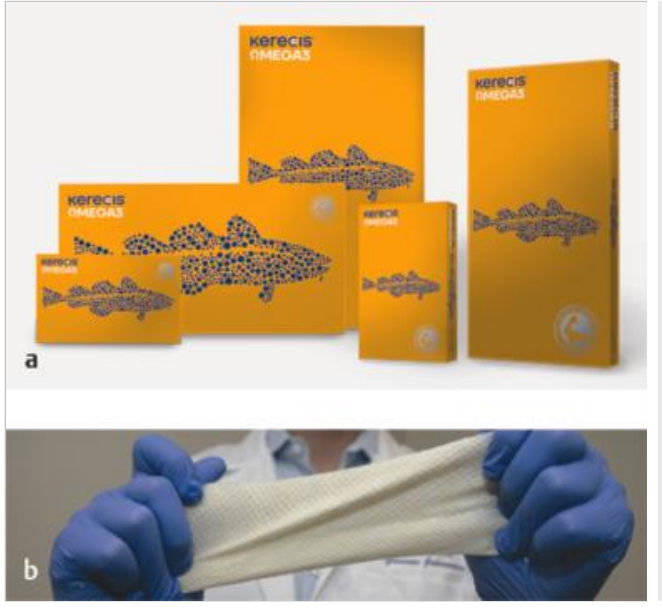

Abb. 2.107 Kerecis. (Quelle: Mit freundlicher Genehmigung von Kerecis)

a Schachtel.

b Gezeigt ist hier die hohe Festigkeit des Materials. 

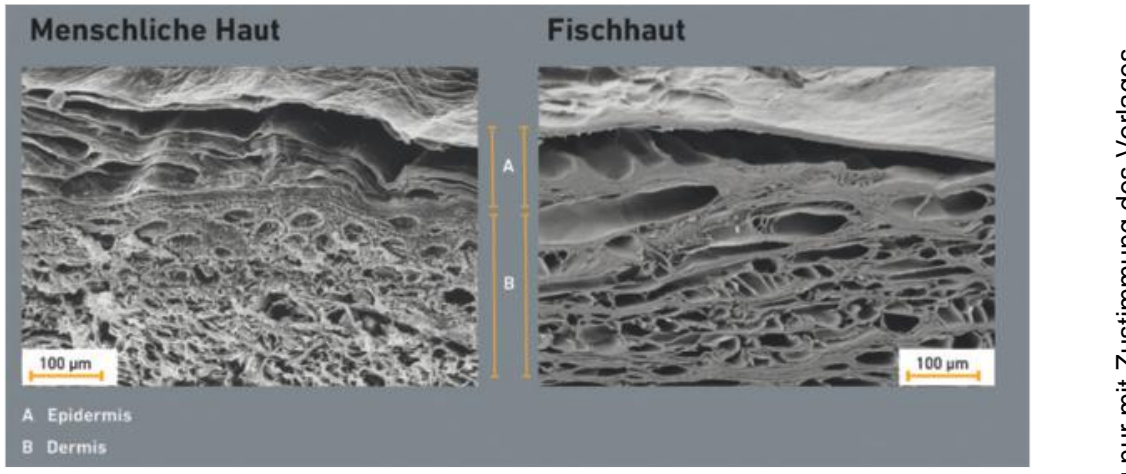

Abb. 2.108 Mikroskopischer Vergleich von menschlicher Haut und Fischhaut. (Quelle: Mit freundlicher Genehmigung von Kerecis)

Das Material kann auch bei umschriebener Entzündung angewendet werden. Es kann mit einer Unterdrucktherapie kombiniert werden.

Übersicht s. \ Abb. 2.109.

\section{Indikation}

- Amputationswunden

- Druckulzera

- diabetische Ulzera

- traumatische Wunden mit freiliegenden Sehnen/Knochen

- chronische Problem-Ulzera.

\section{Kontraindikationen}

Nicht bekannt. 


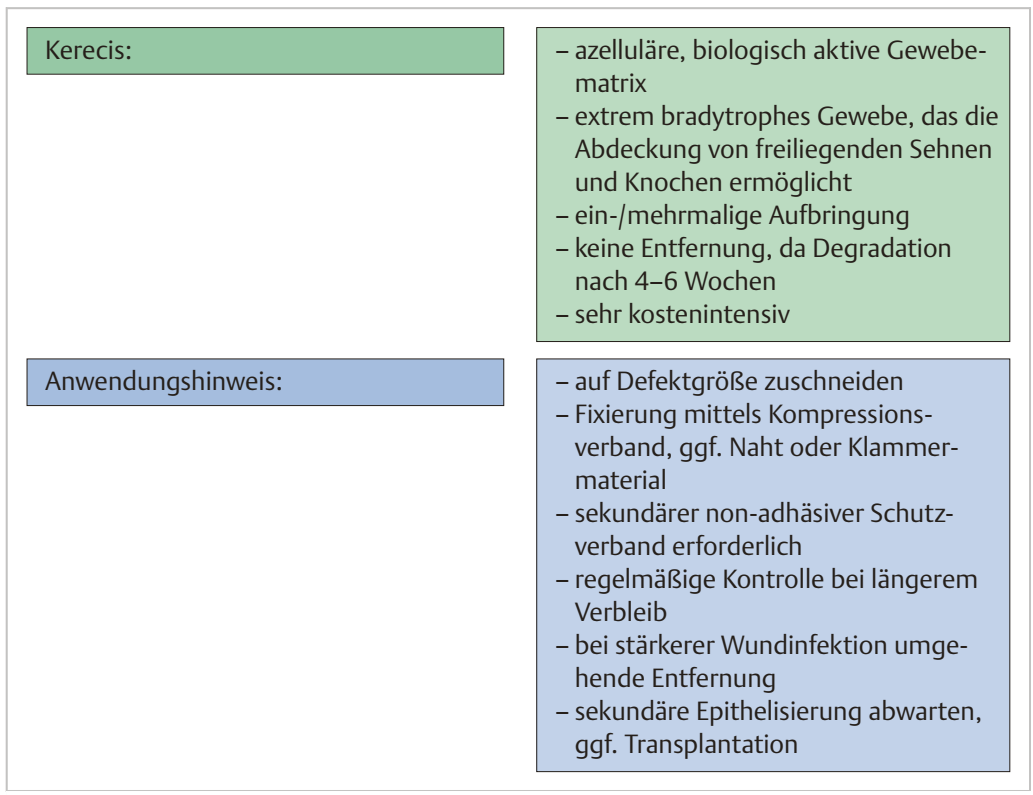

Abb. 2.109 Kerecis.

\section{Anwendungsweise}

Die Fischhaut wird zugeschnitten, in 0,9\% Kochsalzlösung hydratisiert und auf die Wunde aufgelegt ( $\triangleright$ Abb. 2.110). Sie kann mit Nahtmaterial, Klammern etc., falls notwendig, fixiert werden.

Sekundärverband: Als Sekundärverband dient bei exsudierenden Wunden eine flüssigkeitsabsorbierende Wundauflage wie z. B. ein Polyurethanschaum.

Verbandwechsel: Je nach Wundsituation wird nach 1 oder 2 Wochen eine erneute Fischhaut in den Defekt als weiteres Granulationsgerüst eingefügt; die bisherige Matrix wird belassen. Die Häufigkeit des Verbandwechsels hängt von der Exsudatmenge der Wunde ab. 


\section{Wundprodukte}
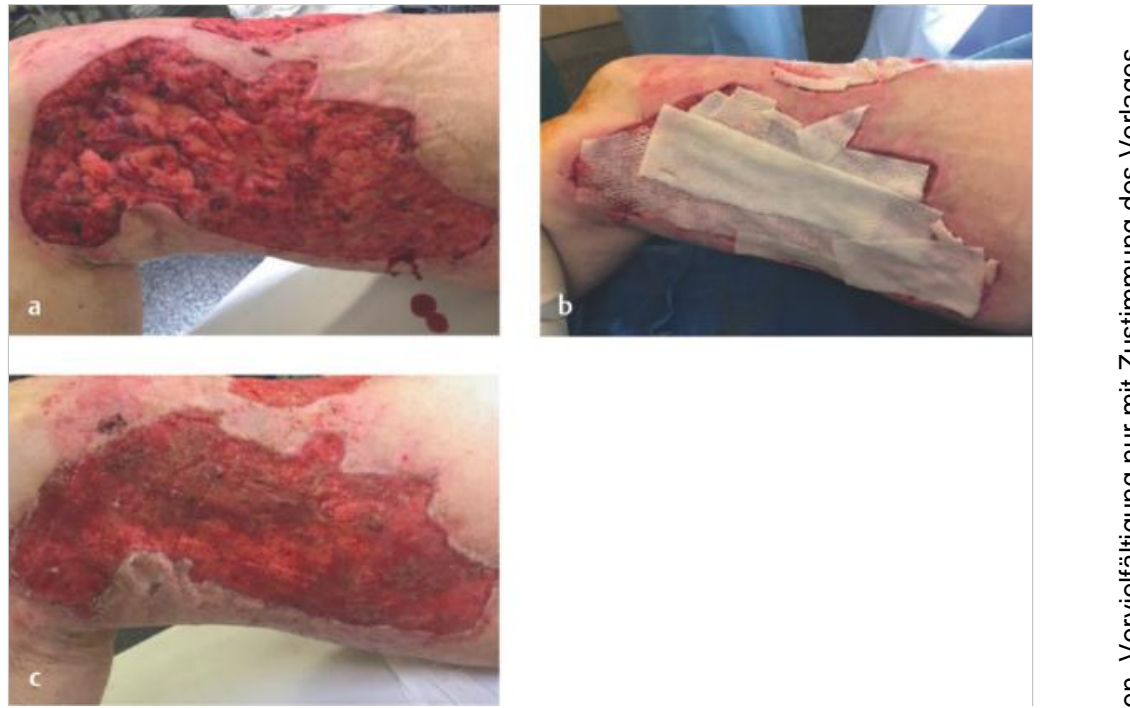

Abb. 2.110 Kerecis: Anwendung. a-c (Quelle: Mit freundlicher Genehmigung von Kerecis) 